\title{
On the uniform perfectness of the groups of diffeomorphisms of even-dimensional manifolds
}

\author{
Takashi Tsuboi *
}

\begin{abstract}
We show that the identity component $\operatorname{Diff}^{r}\left(M^{2 m}\right)_{0}$ of the group of $C^{r}$ diffeomorphisms of a compact (2m)-dimensional manifold $M^{2 m}(1 \leq r \leq \infty, r \neq 2 m+1)$ is uniformly perfect for $2 m \geq 6$, i.e., any element of $\operatorname{Diff}^{r}\left(M^{2 m}\right)_{0}$ can be written as a product of a bounded number of commutators. It is also shown that for a compact connected manifold $M^{2 m}(2 m \geq 6)$, the identity component $\operatorname{Diff}^{r}\left(M^{2 m}\right)_{0}$ of the group of $C^{r}$ diffeomorphisms of $M^{2 m}(1 \leq r \leq \infty, r \neq 2 m+1)$ is uniformly simple, i.e., for elements $f$ and $g$ of $\operatorname{Diff}^{r}\left(M^{2 m}\right)_{0} \backslash\{\mathrm{id}\}, f$ can be written as a product of a bounded number of conjugates of $g$ or $g^{-1}$.
\end{abstract}

Mathematics Subject Classification (2010). Primary 57R52, 57R50; Secondary 37C05.

Keywords. Diffeomorphism group, uniformly perfect, commutator subgroup.

\section{Introduction}

For an $n$-dimensional manifold $M^{n}$, let $\operatorname{Diff}_{c}^{r}\left(M^{n}\right)$ denote the group of $C^{r}$ diffeomorphisms of $M^{n}$ with compact support $(1 \leq r \leq \infty)$. Here, the support of a diffeomorphism $f$ of $M^{n}$ is defined to be the closure of $\{x \in M \mid f(x) \neq x\}$. For a compact manifold $M^{n}$, Diff ${ }_{c}^{r}\left(M^{n}\right)$ coincides with the group $\operatorname{Diff}^{r}\left(M^{n}\right)$ of $C^{r}$ diffeomorphisms of $M^{n}$. Let $\operatorname{Diff}_{c}^{r}\left(M^{n}\right)_{0}$ denote the identity component of $\operatorname{Diff}_{c}^{r}\left(M^{n}\right)$. Here $\operatorname{Diff}_{c}^{r}\left(M^{n}\right)$ is equipped with the $C^{r}$ topology ([16], [23]). By the results of Herman, Mather and Thurston ([11], [14], [16], [23], [2]), for an $n$-dimensional manifold $M^{n}, \operatorname{Diff}_{c}^{r}\left(M^{n}\right)_{0}$ is a perfect group if $r=0$ or $1 \leq r \leq \infty$ and $r \neq n+1$. Here, a group is said to be perfect if it coincides with its commutator subgroup. In other words, a group is perfect if any element can be written as a product of commutators. The perfectness of a group is equivalent to the vanishing of first homology group of the group. The homological properties of the group $\operatorname{Diff}_{c}^{r}\left(M^{n}\right)_{0}$ has been studied in connection with the theory of foliations ([23]).

* The author is partially supported by Grant-in-Aid for Scientific Research (A) 20244003, Grant-in-Aid for Exploratory Research 21654009, Japan Society for Promotion of Science, and by the Global COE Program at Graduate School of Mathematical Sciences, the University of Tokyo. 
In general, for an element $g$ of the commutator subgroup $[G, G]$ of a group $G$, its commutator length is defined to be the minimum number of commutators whose product is equal to $g$. It is natural to ask whether the commutator length function $\mathrm{cl}:[G, G] \rightarrow \mathbb{Z}$ is bounded. When the commutator length is not bounded, then it is very interesting to know about the stable commutator length defined by $\operatorname{scl}(g)=$ $\lim _{n \rightarrow \infty} \mathrm{cl}\left(g^{n}\right) / n$ in Bavard [3]. The stable commutator length function is related with the bounded cohomology groups $H_{b}^{*}(G)$ of the group $G$ defined by Gromov ([7]). Namely, the homomorphism $H_{b}^{2}(G) \rightarrow H^{2}(G)$ is injective if and only if the stable commutator length function vanishes on $[G, G]$. This is formulated as the Bavard duality theorem which describes the stable commutator length in terms of homogeneous quasimorphisms ([3]). In recent years, the stable commutator length and the quasimorphisms appear as an important key to study infinite groups (see for example [5] and its references).

We say that a group is uniformly perfect if any element can be written as a product of a bounded number of commutators. It is easy to see that the uniform perfectness implies the vanishing of stable commutator length function, and hence the injectivity of the map from the second bounded cohomology group to the usual one.

For the question of uniform perfectness of the group of diffeomorphisms, the following results are shown in [4], [30] and [31].

Theorem 1.1 (Burago-Ivanov-Polterovich [4], Tsuboi [30], [31]).

(1) For the interior $M^{n}$ of a compact n-dimensional manifold which admits a handle decomposition only with handles of indices not greater than $(n-1) / 2$, any element of $\operatorname{Diff}_{c}^{r}\left(M^{n}\right)_{0}(1 \leq r \leq \infty, r \neq n+1)$ can be written as a product of two commutators.

(2) For a compact even-dimensional manifold $M^{2 m}$ which has a handle decomposition without handles of the middle index $m$, any element of $\operatorname{Diff}^{r}\left(M^{2 m}\right)_{0}$ $(1 \leq r \leq \infty, r \neq 2 m+1)$ can be written as a product of four commutators.

(3) For an arbitrary compact odd-dimensional manifold $M^{2 m+1}$, any element of $\operatorname{Diff}^{r}\left(M^{2 m+1}\right)_{0}(1 \leq r \leq \infty, r \neq 2 m+2)$ can be written as a product of five commutators.

Now the result of this paper concerns the remaining cases.

Theorem 1.2. The identity component $\operatorname{Diff}^{r}\left(M^{2 m}\right)_{0}$ of the group of $C^{r}$ diffeomorphisms $\operatorname{Diff}^{r}\left(M^{2 m}\right)$ of the compact $(2 m)$-dimensional manifold $M^{2 m}(1 \leq r \leq \infty$, $r \neq 2 m+1)$ is uniformly perfect for $2 m \geq 6$, i.e., any element of $\operatorname{Diff}^{r}\left(M^{2 m}\right)_{0}$ can be written as a product of a bounded number of commutators.

Here the bound for the number of commutators may depend on manifolds. For the manifolds of dimensions 2 and 4, the problem of uniform perfectness of the 
identity component of the group of diffeomorphisms is still open. The vanishing of the stable commutator length of these groups is not known either. It is interesting to find some other approach to study the stable commutator length of diffeomorphism groups which might solve the remaining cases (see [4], [12]).

The argument deducing the simplicity of $\operatorname{Diff}^{r}\left(M^{n}\right)_{0}$ from the proof of its perfectness ([8], [23], [2]) applies to showing the uniform simplicity from the proof of its uniformly perfectness ([31]). We say that a group $G$ is uniformly simple if, for elements $f$ and $g$ of $G \backslash\{\mathbf{1}\}, f$ can be written as a product of a bounded number of conjugates of $g$ or $g^{-1}$.

Corollary 1.3. For a compact connected $(2 m)$-dimensional manifold $M^{2 m}(2 m \geq$ $6)$, the identity component $\operatorname{Diff}^{r}\left(M^{2 m}\right)_{0}$ of the group $\operatorname{Diff}^{r}\left(M^{2 m}\right)$ of $C^{r}$ diffeomorphisms of $M^{2 m}(1 \leq r \leq \infty, r \neq 2 m+1)$ is uniformly simple.

The main part of the proof of Theorem 1.2 is a decomposition of an isotopy into a bounded number of isotopies with controlled support. Then the theorem follows from Theorem 1.1(1) in a way similar to the proof of Theorem 1.1(2) and (3) in [30] and in [31]. For the decomposition, we give a technique to find the Whitney disks which guide to separate two stratified subsets of the middle dimension $m$. The condition $2 m \geq 6$ on the dimension implies that the Whitney disks can be disjointly embedded in the manifold and enables us to show Theorem 1.2.

We review the proof of Theorem 1.1 in Section 2 and there we give lemmas about the general position of two stratified subsets which were not correctly stated in [31]. Then we give the proof of Theorem 1.2 in Section 3. The proof of lemmas used in Section 3 is given in Sections 4 and 6. We show Corollary 1.3 in Section 5.

The author is grateful to the referee for patient and careful reading and for pointing out several errors in the earlier versions, one of which is a misleading statement on relationship between the decomposition by the stable manifolds of a gradient flow of a Morse function and a cellular decomposition of the manifold (see Section 6).

\section{Decomposition of isotopies}

The proof of our Theorem 1.2 relies on the general position argument for differentiable maps between manifolds with stratified subsets. In [30] and [31], we looked at the general position of the differentiable mappings from a cellular complex to a manifold with differentiable cellular decomposition.

The argument in [30] and [31] works for differentiable manifolds with stratified subsets which are defined as follows: Let $M^{n}$ be an $n$-dimensional manifold. A subset $X$ of $M^{n}$ is an $m$-dimensional stratified subset if there is a filtration

$$
X=X^{(m)} \supset X^{(m-1)} \supset \cdots \supset X^{(1)} \supset X^{(0)},
$$


such that, for $k=0, \ldots, m$,

(1) $X^{(k)}$ is a closed subset,

(2) $X^{(k)} \backslash X^{(k-1)}$ is a $k$-dimensional submanifold of $M^{n}$,

(3) for the closure $\overline{X^{(k)} \backslash X^{(k-1)}}$ of $X^{(k)} \backslash X^{(k-1)}$,

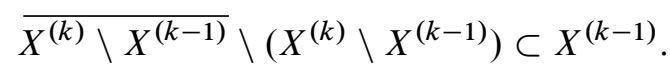

The subset $X^{(k)}$ is called the $k$-dimensional skeleton of $X$. This definition of the stratified subsets is a weak one ([36], [24]).

First we show the following lemma which is the necessary generalization of Lemma 4.3 in [30] or Lemma 2.3 in [31].

Lemma 2.1. Let $M^{n}$ be an $n$-dimensional manifold with a compact $k$-dimensional stratified subset $K^{k}$, and $N^{m}$ be an $m$-dimensional manifold with a compact $\ell$ dimensional stratified subset $L^{\ell}$. Let $f: N^{m} \rightarrow M^{n}$ be a differentiable map. If $k+\ell+1 \leq n$, then there is an isotopy $\left\{\Phi_{t}: M^{n} \rightarrow M^{n}\right\}_{t \in[0,1]}\left(\Phi_{0}=\mathrm{id}\right)$ such that $\Phi_{1}\left(K^{k}\right) \cap f\left(L^{\ell}\right)=\emptyset$.

Proof. We construct the isotopy $\Phi_{t}$, skeleton by skeleton. Let $K^{(u)}$ denote the $u$ dimensional skeleton of $K^{k}$;

$$
K^{k}=K^{(k)} \supset \cdots \supset K^{(1)} \supset K^{(0)} .
$$

Assume that for $u-1 \leq k-1$, there is an isotopy $\left\{\Phi_{t}^{u-1}\right\}_{t \in[0,1]}\left(\Phi_{0}^{u-1}=\mathrm{id}\right)$ such that

$$
\Phi_{1}^{u-1}\left(K^{(u-1)}\right) \cap f\left(L^{\ell}\right)=\emptyset .
$$

Then there is a neighborhood $U_{u-1}$ of $K^{(u-1)}$ such that $\Phi_{1}^{u-1}\left(U_{u-1}\right) \cap f\left(L^{\ell}\right)=\emptyset$.

Now for $u \leq k$, we construct an isotopy $\left\{\Phi_{t}^{u}\right\}_{t \in[0,1]}\left(\Phi_{0}^{u}=\right.$ id) such that $\Phi_{1}^{u}\left(K^{(u)}\right) \cap f\left(L^{\ell}\right)=\emptyset$. Since $K^{(u)}$ is closed in $K^{k}, K^{(u)} \backslash U_{u-1}$ is compact and is covered by finitely many coordinate neighborhoods $\left\{\left(D^{u} \times D^{n-u}\right)_{i}\right\}_{i=1}^{k_{u}}$ of $M^{n}$ of the form $D^{u} \times D^{n-u}$, where $D^{u}$ and $D^{n-u}$ are the closed balls of radius 1 in $\mathbb{R}^{u}$ and $\mathbb{R}^{n-u}$, respectively, and

$$
\left(K^{(u)} \backslash U_{u-1}\right) \cap\left(D^{u} \times D^{n-u}\right)_{i} \subset\left(D^{u} \times\{0\}\right)_{i} .
$$

Moreover we can take such neighborhoods that the family

$$
\left\{\left(\operatorname{int}\left(D_{1 / 2}^{u}\right) \times \operatorname{int}\left(D_{1 / 2}^{n-u}\right)\right)_{i}\right\}_{i=1}^{k_{u}}
$$

still covers $K^{(u)} \backslash U_{u-1}$, and

$$
K^{(u)} \backslash U_{u-1} \subset \bigcup_{i=1}^{k_{u}}\left(\operatorname{int}\left(D_{1 / 2}^{u}\right) \times\{0\}\right)_{i},
$$


where $D_{1 / 2}^{u}$ and $D_{1 / 2}^{n-u}$ are the images of the closed balls of radius $1 / 2$ in $\mathbb{R}^{u}$ and $\mathbb{R}^{n-u}$, respectively, and "int" denotes the interior.

Now assume that for $i-1 \leq k_{u}-1$, we have an isotopy $\left\{\Phi_{t}^{u, i-1}\right\}_{t \in[0,1]}\left(\Phi_{0}^{u, i-1}=\right.$ id) with support in $\bigcup_{j=1}^{i-1}\left(D^{u} \times D^{n-u}\right)_{j}$ such that

$$
K^{(u)} \cap\left(\Phi_{1}^{u-1} \circ \Phi_{1}^{u, i-1}\right)^{-1}\left(f\left(L^{\ell}\right)\right) \subset \bigcup_{j=i}^{k_{u}}\left(\operatorname{int}\left(D_{1 / 2}^{u}\right) \times \operatorname{int}\left(D_{1 / 2}^{n-u}\right)\right)_{j} .
$$

On the neighborhood $\left(D^{u} \times D^{n-u}\right)_{i}$, we have the projection

$$
p_{i}=\operatorname{proj}_{2}:\left(D^{u} \times D^{n-u}\right)_{i} \longrightarrow D^{n-u} .
$$

Put $L_{i-1}^{\ell}=\left(\Phi_{1}^{u-1} \circ \Phi_{1}^{u, i-1}\right)^{-1}\left(f\left(L^{\ell}\right)\right)$. Since $p_{i}\left(L_{i-1}^{\ell} \cap\left(D^{u} \times D^{n-u}\right)_{i}\right)$ is a finite union of images of manifolds of dimension $\leq \ell \leq n-k-1 \leq n-u-1$ under differentiable maps of class $C^{r}(r \geq 1)$, it is a measure zero subset of $D^{n-u}$ by the Sard theorem. Moreover, since $\bar{L}^{\ell}$ is compact, $p_{i}\left(L_{i-1}^{\ell} \cap\left(D^{u} \times D^{n-u}\right)_{i}\right)$ is a nowhere dense closed subset of $D^{n-u}$. Take a point $q_{i}$ close to 0 in the complement of $p_{i}\left(L_{i-1}^{\ell} \cap\left(D^{u} \times D^{n-u}\right)_{i}\right)$. Let $\left\{\Phi_{t}^{\prime u, i}: M^{n} \rightarrow M^{n}\right\}_{t \in[0,1]}\left(\Phi_{0}^{\prime u, i}=\right.$ id $)$ be an isotopy with support in $\left(\operatorname{int}\left(D^{u}\right) \times \operatorname{int}\left(D^{n-u}\right)\right)_{i}$ such that $\Phi_{t}^{\prime u, i}(x, 0)=\left(x, t \mu(x) q_{i}\right)$ on $\left(D^{u} \times D^{n-u}\right)_{i}$, where $\mu: \operatorname{int}\left(D^{u}\right) \rightarrow[0,1]$ is a $C^{\infty}$ function with compact support such that $\mu(x)=1$ for $x \in D_{1 / 2}^{u}$. Since we took $q_{i}$ in the complement of $p_{i}\left(L_{i-1}^{\ell} \cap\left(D^{u} \times D^{n-u}\right)_{i}\right)$,

$$
L_{i-1}^{\ell} \cap \Phi_{1}^{\prime u, i}\left(K^{(u)}\right) \cap\left(\operatorname{int}\left(D_{1 / 2}^{u}\right) \times \operatorname{int}\left(D_{1 / 2}^{n-u}\right)\right)_{i}=\emptyset,
$$

hence

$$
\left(\Phi_{1}^{\prime u, i}\right)^{-1}\left(L_{i-1}^{\ell}\right) \cap K^{(u)} \cap\left(\operatorname{int}\left(D_{1 / 2}^{u}\right) \times \operatorname{int}\left(D_{1 / 2}^{n-u}\right)\right)_{i}=\emptyset .
$$

Since we took $q_{i}$ sufficiently close to $0 \in D^{n-u}$,

$$
\left(\Phi_{t}^{\prime u, i}\right)^{-1}\left(L_{i-1}^{\ell}\right) \cap\left(K^{(u)} \cup \bigcup_{j=1}^{i-1}\left(\operatorname{int}\left(D_{1 / 2}^{u}\right) \times \operatorname{int}\left(D_{1 / 2}^{n-u}\right)\right)_{j}\right)=\emptyset(t \in[0,1]) .
$$

Thus we found the isotopy $\left\{\Phi_{t}^{u, i}=\Phi_{t}^{u, i-1} \circ \Phi_{t}^{\prime u, i}\right\}_{t \in[0,1]}\left(\Phi_{0}{ }^{u, i}=\mathrm{id}\right)$ with support in $\bigcup_{j=1}^{i}\left(D^{u} \times D^{n-u}\right)_{j}$ such that

$$
K^{(u)} \cap\left(\Phi_{1}^{u-1} \circ \Phi_{1}^{u, i}\right)^{-1}\left(f\left(L^{\ell}\right)\right) \subset \bigcup_{j=i+1}^{k_{u}}\left(\operatorname{int}\left(D_{1 / 2}^{u}\right) \times \operatorname{int}\left(D_{1 / 2}^{n-u}\right)\right)_{j} .
$$

Let $\Phi_{t}^{u}$ be the composition $\Phi_{t}^{u-1} \circ \Phi_{t}^{u, k_{u}}$, then $\left\{\Phi_{t}^{u}\right\}_{t \in[0,1]}\left(\Phi_{0}^{u}=\mathrm{id}\right)$ satisfies that $\Phi_{1}^{u}\left(K^{(u)}\right) \cap f\left(L^{\ell}\right)=\emptyset$. Then $\Phi_{t}=\Phi_{t}^{k}$ satisfies $\Phi_{1}\left(K^{k}\right) \cap f\left(L^{\ell}\right)=\emptyset$. 
We use Lemma 2.1 to show the following theorem ([30], [31]).

Theorem 2.2. Let $M^{n}$ be a compact $n$-dimensional manifold. Let $P^{p}$ and $Q^{q}$ be p-dimensional and q-dimensional stratified subsets in $M^{n}$, respectively. Assume that $p+q+2 \leq n$ and that $P^{p} \cap Q^{q}=\emptyset$. Then any element $f \in \operatorname{Diff}^{r}\left(M^{n}\right)_{0}$ $(1 \leq r \leq \infty)$ can be written as a product $f=$ goh such that $g \in \operatorname{Diff}_{c}^{r}\left(M^{n} \backslash k\left(Q^{q}\right)\right)_{0}$ and $h \in \operatorname{Diff}_{c}^{r}\left(M^{n} \backslash P^{p}\right)_{0}$, where $k \in \operatorname{Diff}_{c}^{r}\left(M^{n} \backslash P^{p}\right)_{0}$ is a diffeomorphism of $M^{n}$ with support in a small neighborhood of $Q^{q}$, and $\operatorname{Diff}_{c}^{r}\left(M^{n} \backslash k\left(Q^{q}\right)\right)_{0}$ and $\operatorname{Diff}_{c}^{r}\left(M^{n} \backslash P^{p}\right)_{0}$ are considered as subgroups of $\operatorname{Diff}^{r}\left(M^{n}\right)_{0}$, respectively.

The statement of Theorem 2.2 means that, by moving $Q$ by a small isotopy $k$, the diffeomorphism $g$ of $M^{n}$ obtained in Theorem 2.2 is isotopic to the identity by an isotopy which is the identity on a neighborhood of $k\left(Q^{q}\right)$, and $h$ is isotopic to the identity by an isotopy which is the identity on a neighborhood of $P^{p}$.

For the completeness, we include the proof of Theorem 2.2.

Proof of Theorem 2.2. Let $\left\{f_{t}\right\}_{t \in[0,1]}$ be the isotopy such that $f_{0}=\mathrm{id}$ and $f_{1}=f$. Let $F:[0,1] \times M^{n} \rightarrow M^{n}$ be the trace of the isotopy: $F(t, x)=f_{t}(x)$. Here, $[0,1] \times M^{n}$ contains the $(p+1)$-dimensional stratified subset $[0,1] \times P^{p}$.

We look at the image $F\left([0,1] \times P^{p}\right) \subset M^{n}$. As $p+1+q \leq n-1$, by Lemma 2.1 , there is an isotopy $\left\{k_{s}\right\}_{s \in[0,1]}\left(k_{0}=\mathrm{id}, k_{1}=k\right)$ such that $F\left([0,1] \times P^{p}\right) \cap$ $k\left(Q^{q}\right)=\emptyset$.

Then the following lemma implies Theorem 2.2 by putting $P_{0}=\emptyset$ and replacing $Q^{q}$ by $k\left(Q^{q}\right)$.

Lemma 2.3. Let $M^{n}$ be a compact $n$-dimensional manifold. Let $P^{p}$ and $Q^{q}$ be p-dimensional and $q$-dimensional stratified subsets of $M^{n}$, respectively. Let $P_{0}$ be a subset of $P^{p}$. Let $\left\{f_{t}\right\} \subset \operatorname{Diff}^{r}\left(M^{n}\right)_{0}\left(f_{0}=\mathrm{id}\right)$ be an isotopy which is the identity on a neighborhood of $P_{0}$. Assume that $f_{t}\left(P^{p} \backslash P_{0}\right) \cap Q^{q}=\emptyset(t \in[0,1])$. Then $f_{1} \in \operatorname{Diff}^{r}\left(M^{n}\right)_{0}$ can be written as a product $f_{1}=g_{1} \circ h_{1}$, where $\left\{g_{t}\right\}_{t \in[0,1]} \subset$ $\operatorname{Diff}_{c}^{r}\left(M^{n} \backslash Q^{q}\right)_{0}\left(g_{0}=\mathrm{id}\right)$ and $\left\{h_{t}\right\}_{t \in[0,1]} \subset \operatorname{Diff}_{c}^{r}\left(M^{n} \backslash P^{p}\right)_{0}\left(h_{0}=\mathrm{id}\right)$.

Proof. Let $F:[0,1] \times M^{n} \rightarrow M^{n}$ be the trace of the isotopy: $F(t, x)=f_{t}(x)$.

Let $W$ be a neighborhood of $P_{0}$ in $M^{n}$ where $f_{t}$ is the identity. Let $U$ be a neighborhood of $F\left([0,1] \times\left(P^{p} \backslash W \cap P^{p}\right)\right)$ and $V$ be a neighborhood of $Q^{q}$ such that $U \cap V=\emptyset$.

Let $\xi$ be the vector field on $[0,1] \times M^{n}$ given by $\frac{\partial}{\partial t}+\left(\frac{\mathrm{d} f_{t+s}(x)}{\mathrm{d} s}\right)_{s=0}$ at $\left(t, f_{t}(x)\right)$. This $\xi$ generates the isotopy $f_{t}$. Let $\eta$ be a vector field on $[0,1] \times M^{n}$ with support in $[0,1] \times U$ such that $\eta=\xi$ on a neighborhood of

$$
\left\{\left(t, f_{t}\left(x_{0}\right)\right) \mid x_{0} \in P^{p} \backslash W \cap P^{p}, t \in[0,1]\right\} .
$$


Then $\eta=\partial / \partial t$ on $[0,1] \times(V \cup W)$ which is a neighborhood of $[0,1] \times\left(Q^{q} \cup P_{0}\right)$. Then $\eta$ generates an isotopy $\left\{g_{t}\right\}_{t \in[0,1]}$ such that $g_{t}$ is the identity on the neighborhood $V \cup W$ of $Q^{q} \cup P_{0}$ and $g_{t}(x)=f_{t}(x)$ for $x$ in a neighborhood of $P^{p}=\left(P^{p} \backslash W \cap\right.$ $\left.P^{p}\right) \cup\left(W \cap P^{p}\right)$. Here, for $x \in W, g_{t}(x)=x=f_{t}(x)$. Put $h=g_{1}{ }^{-1} f_{1}$, then $h$ is the identity on a neighborhood of $P^{p}$, and it is isotopic to the identity as an element of $\operatorname{Diff}^{r}\left(M^{n}\right)$. For, put $h_{t}=g_{t}{ }^{-1} \circ f_{t}$. Then $h_{t}$ is the identity on a neighborhood of $P^{p}$.

Thus we can write $f=g \circ h$, where $g \in \operatorname{Diff}_{c}^{r}\left(M^{n} \backslash Q^{q}\right)_{0}, h \in \operatorname{Diff}_{c}^{r}\left(M^{n} \backslash P^{p}\right)_{0}$.

To use Theorem 2.2, we looked at the stratifications of a compact manifold $M^{n}$ given by the stable manifolds or by the unstable manifolds of the gradient flow of a Morse function associated with a handle decomposition.

A function $f: M^{n} \rightarrow \mathbb{R}$ on a compact $n$-dimensional manifold $M^{n}$ without boundary is called a Morse function if the critical points are nondegenerate, that is, the Hessian matrices of $f$ at the critical points are nondegenerate. For such a function $f$, the set of critical points is a finite set. The index of the Hessian matrix of $f$ at a critical point is called the index of the critical point.

Any compact $n$-dimensional manifold $M^{n}$ without boundary admits a Morse function $f: M^{n} \rightarrow \mathbb{R}$ such that $f\left(M^{n}\right)=[0, n]$, the set of critical points of index $k$ is contained in $f^{-1}(k)(k=0, \ldots, n)$. Such a Morse function is called self-indexing. If $M^{n}$ is a compact connected $n$-dimensional manifold $M^{n}$ without boundary, there is a self-indexing Morse function $f: M^{n} \rightarrow \mathbb{R}$ such that $f^{-1}(0)$ and $f^{-1}(n)$ are one point sets ([19]).

For $a \in[0, n]$, put $M_{a}=f^{-1}(a)$. Then $M_{a}$ is a codimension 1 submanifold of $M^{n}$ if $a$ is not an integer. Put $W_{k}=f^{-1}([0, k+1 / 2])$, and then this $W_{k}$ is a compact manifold with boundary $\partial W_{k}=M_{k+1 / 2}=f^{-1}(k+1 / 2)$. Let $c_{k}$ be the number of critical points of index $k$. Then the manifold $W_{k}$ is diffeomorphic to the manifold obtained from $W_{k-1}$ by attaching $c_{k}$ handles of index $k(k=0, \ldots, n)$. This means the following.

Let $D^{k} \times D^{n-k}$ be the product of the $k$-dimensional disk $D^{k}$ and the $(n-k)$ dimensional disk $D^{n-k}$. Let $\varphi_{i}:\left(\partial D^{k}\right) \times D^{n-k} \rightarrow \partial W_{k-1}\left(i=1, \ldots, c_{k}\right)$ be diffeomorphisms with disjoint images. Let

$$
W_{k}^{\prime}=W_{k-1} \cup_{\bigsqcup_{i=1}^{c_{k}} \varphi_{i}} \bigsqcup_{i=1}^{c_{k}}\left(D^{k} \times D^{n-k}\right)_{i}
$$

be the space obtained from the disjoint union $W_{k-1} \sqcup \bigsqcup_{i=1}^{c_{k}}\left(D^{k} \times D^{n-k}\right)_{i}$ by identifying $x \in\left(\partial D^{k}\right) \times D^{n-k} \subset\left(D^{k} \times D^{n-k}\right)_{i}$ with $\varphi_{i}(x) \in \partial W_{k-1} \subset W_{k-1}$. The image of $D^{k} \times D^{n-k}$ in $W_{k}^{\prime}$ is called a handle of index $k$. We will simply write the handle of index $k$ as $\left(D^{k} \times D^{n-k}\right)_{i}$. Then $W_{k}^{\prime}$ is a manifold with boundary and the corner which is the image $\bigsqcup_{i=1}^{c_{k}} \varphi_{i}\left(\left(\partial D^{k}\right) \times\left(\partial D^{n-k}\right)\right)$. By smoothing along the corner, we 
obtain $W_{k}^{\prime \prime}$ from $W_{k}^{\prime}$ and $W_{k}^{\prime \prime}$ has a differentiable structure which is diffeomorphic to $W_{k}$, and we say $W_{k}$ is obtained from the manifold $W_{k-1}$ by attaching $c_{k}$ handles of index $k(k=0, \ldots, n)$.

In fact, we can consider $W_{k}^{\prime}$ as a submanifold with corner of $W_{k}, W_{k}^{\prime \prime}$ is obtained by taking the union of $W_{k}^{\prime}$ and a neighborhood of corner of $W_{k}^{\prime}$, and $W_{k} \backslash W_{k}^{\prime \prime}$ is diffeomorphic to $(-\infty, k+1 / 2] \times \partial W_{k}$. We have the sequence of submanifolds

$$
\begin{aligned}
W_{0} \subset W_{1}^{\prime} \subset W_{1}^{\prime \prime} \subset W_{1} \subset \cdots & \subset W_{k-1} \subset W_{k}^{\prime} \subset W_{k}^{\prime \prime} \subset W_{k} \\
\subset \cdots & \subset W_{n-1} \subset W_{n}^{\prime}=W_{n}^{\prime \prime}=W_{n}=M^{n} .
\end{aligned}
$$

Then, when we identify $W_{k}^{\prime}$ with $W_{k}, M^{n}$ is decomposed into the union of the handles $\left(D^{k} \times D^{n-k}\right)_{i}\left(i=1, \ldots, c_{k} ; k=0, \ldots, n\right)$ and this decomposition into handles is called a handle decomposition of $M$. However, hereafter we do not identify $W_{k}^{\prime}$ or $W_{k}^{\prime \prime}$ with $W_{k}$. We call the image of $D^{k} \times\{0\}$ the core disk of the handle $\left(D^{k} \times D^{n-k}\right)_{i}$ of index $k$. The boundary of the core disk of the handle of index $k$ is an embedded $(k-1)$-dimensional sphere in $\partial W_{k-1}=M_{k-1 / 2}$ and it is called the attaching sphere.

For the above self-indexing Morse function $f: M^{n} \rightarrow \mathbb{R}$ and the constant function $n$, the function $n-f$ is a Morse function, and the critical points of index $k$ of the Morse function $f$ are nothing but the critical points of index $n-k$ of the Morse function $n-f$. Hence this gives rise to a handle decomposition of $M^{n}$ called the dual handle decomposition. That is for

$$
\begin{aligned}
W_{n-k}^{*}= & (n-f)^{-1}([0, n-k+1 / 2])=f^{-1}([k-1 / 2, n]), \\
M^{n} & =W_{n}^{*}=W_{n}^{* \prime \prime}=W_{n}^{* \prime} \supset W_{n-1}^{*} \\
& \supset \cdots \supset W_{n-k}^{*} \supset W_{n-k}^{* \prime \prime} \supset W_{n-k}^{* \prime} \supset W_{n-k-1}^{*} \\
& \supset \cdots \supset W_{1}^{*} \supset W_{1}^{* \prime \prime} \supset W_{1}^{* \prime} \supset W_{0}^{*} .
\end{aligned}
$$

Then $W_{n-k}^{* \prime}$ is obtained from $W_{n-k-1}^{*}$ by attaching $c_{k}$ handles of index $n-k$. The core disk of the handle of index $n-k$ for this handle decomposition is called the cocore disk of the handle decomposition for $f$. The boundary of the cocore disk of the handle of index $k$ is an embedded $(n-k-1)$-dimensional sphere in $\partial W_{n-k-1}^{*}=$ $\partial W_{k}=M_{k+1 / 2}$ and it is called the belt sphere.

By choosing a Riemannian metric on the manifold $M^{n}$, the Morse function $f$ defines the gradient vector field and the gradient flow $\Psi_{t}$. The singular points of the gradient vector field are precisely the critical points of $f$. The local stable manifold and the local unstable manifold of the singular point $p$ of the gradient flow $\Psi_{t}$ correspond to the core disk and the cocore disk of the handle containing $p$ of a handle decomposition of $M^{n}$, respectively ([18], [19]). Let $e_{i}^{k}$ and $e_{i}^{* n-k}$ denote the global stable manifold and the global unstable manifold, respectively, for the singular point $p_{i}^{k}$ which is a critical point of index $k$ of $f\left(i=1, \ldots, c_{k}\right)$. Then $e_{i}^{k}$ and $e_{i}^{* n-k}$ are 
diffeomorphic to $\mathbb{R}^{k}$ and $\mathbb{R}^{n-k}$, respectively. Let

$$
X^{(k)}=\bigcup_{j \leq k} \bigcup_{i=1}^{c_{j}} e_{i}^{j} \quad(k=0, \ldots, n) .
$$

Then

$$
M^{n}=X^{(n)} \supset X^{(n-1)} \supset \cdots \supset X^{(1)} \supset X^{(0)}
$$

is a stratification of $M^{n}$ ([18]). That is, $X^{(k)}$ is a closed subset, $\bigcup_{i=1}^{c_{k}} e_{i}^{k}$ is a $k$ dimensional submanifold, and $\overline{\bigcup_{i=1}^{c_{k}} e_{i}^{k}} \backslash \bigcup_{i=1}^{c_{k}} e_{i}^{k} \subset X^{(k-1)}$. We call this the stratification by the stable manifolds (for the gradient flow of the Morse function). We also have the stratification by the unstable manifolds (for the gradient flow of the Morse function):

$$
M^{n}=X^{*(n)} \supset X^{*(n-1)} \supset \cdots \supset X^{*(1)} \supset X^{*(0)},
$$

where $X^{*(n-k)}=\bigcup_{j \geq k} \bigcup_{i=1}^{c_{j}} e_{i}^{* n-j}(k=0, \ldots, n)$. This is the stratification by the stable manifolds for the gradient flow of the Morse function $n-f$.

We look at the $k$-dimensional skeleton $X^{(k)}$ of the stratification by the stable manifolds and the $(n-k-1)$-dimensional skeleton $X^{*(n-k-1)}$ of the stratification by the unstable manifolds. The boundary $\partial W_{k}=M_{k+1 / 2}$ of $W_{k}$ is transverse to the gradient flow $\Psi_{t}$, and hence $M \backslash\left(X^{(k)} \cup X^{*(n-k-1)}\right)$ is diffeomorphic to $\partial W_{k} \times \mathbb{R}$ by the map

$$
\partial W_{k} \times \mathbb{R} \ni(x, t) \longmapsto \Psi_{t}(x) \in M \backslash\left(X^{(k)} \cup X^{*(n-k-1)}\right) .
$$

Moreover $\Psi_{t}\left(\partial W_{k}\right)$ converges to $X^{(k)}$ as $t \rightarrow-\infty$ and to $X^{*(n-k-1)}$ as $t \rightarrow \infty$. Hence, $M \backslash X^{*(n-k-1)}$ is diffeomorphic to the interior $\operatorname{int}\left(W_{k}\right)$ of $W_{k}$, and any small neighborhood of $X^{(k)}$ contains a deformation retract of both $W_{k}$ and $M \backslash X^{*(n-k-1)}$ :

$$
X^{(k)} \subset \operatorname{int}\left(W_{k}\right) \subset W_{k} \subset M \backslash X^{*(n-k-1)} .
$$

Using the gradient flow $\Psi_{t}$, for any neighborhood $V$ of $X^{(k)} \operatorname{in} \operatorname{int}\left(W_{k}\right)$ and for any compact subset $A$ in $\operatorname{int}\left(W_{k}\right)$, we can construct an isotopy $\left\{G_{t}: \operatorname{int}\left(W_{k}\right) \rightarrow\right.$ $\left.\operatorname{int}\left(W_{k}\right)\right\}_{t \in[0,1]}$ with compact support such that $G_{0}=\operatorname{id}_{\text {int }\left(W_{k}\right)}, G_{t}\left(X^{(k)}\right) \subset X^{(k)}$ $(t \in[0,1])$ and $G_{1}(A) \subset V$. A similar statement is true for $X^{(k)} \subset M \backslash X^{*(n-k-1)}$.

Remark 2.4. For our Morse function there is a Riemannian metric on $M^{n}$ such that the stable manifolds $e_{i}^{k}$ and the unstable manifolds $e_{i^{\prime}}^{* k^{\prime}}$ intersect transversely ([21]). As we shall see in Section 6 (Proposition 6.2), for a carefully chosen Riemannian metric, there is a cellular complex structure compatible with the stratification by stable manifolds.

Now for the interior $M^{n}$ of a compact manifold with boundary $\bar{M}^{n}$ which admits a Morse function such that $W_{m}=\bar{M}^{n}$ for $2 m<n$, we have the following lemma (see [30], Lemma 4.5). 
Lemma 2.5. Let $M^{n}$ be the interior of a compact $n$-dimensional manifold which admits a handle decomposition only with handles of indices not greater than $(n-1) / 2$. Let $X^{(m)}$ be the $m$-dimensional skeleton of the stratification by the stable manifolds for the gradient flow of the Morse function on $M^{n}$ adapted to the handle decomposition $(2 m<n)$. Then there are an isotopy $\left\{F_{t}: M^{n} \rightarrow M^{n}\right\}_{t \in[0,1]}$ with compact support $\left(F_{0}=\mathrm{id}\right)$ and an open neighborhood $U$ of $X^{(m)}$ such that $\left(F_{1}\right)^{\ell}(U)(\ell \in \mathbb{Z})$ are disjoint.

Proof. Let $V_{0}$ be a small neighborhood of $X^{(m)} \subset M^{n}$. We apply Lemma 2.1 to the identity map $M^{n} \rightarrow M^{n}$ of $M^{n}$ with stratified subset $X^{(m)}$. Then there is an isotopy $\left\{h_{t}\right\}_{t \in[0,1]}$ such that $h_{0}=$ id and $h_{1}\left(X^{(m)}\right) \cap X^{(m)}=\emptyset$. We may assume that the support of the isotopy $\left\{h_{t}\right\}_{t \in[0,1]}$ is contained in $V_{0}$. Take a neighborhood $V_{1}$ of $X^{(m)}$ and $V_{2}$ of $h_{1}\left(X^{(m)}\right)$ such that $V_{1} \cap V_{2}=\emptyset$. Then $V_{3}=V_{1} \cap\left(h_{1}\right)^{-1}\left(V_{2}\right)$ is a neighborhood of $X^{(m)}$ such that $V_{3} \cap h_{1}\left(V_{3}\right)=\emptyset$. Here we can take $V_{1}$ and $V_{2}$ such that their closures $\bar{V}_{1}$ and $\bar{V}_{2}$ are compact, and then $\bar{V}_{3}$ is compact.

For $V_{3}$ and $h_{1}\left(\overline{V_{3}}\right)$, by using the flow lines of the gradient flow $\Psi_{t}$, we have an isotopy $\left\{G_{t}: M^{n} \rightarrow M^{n}\right\}_{t \in[0,1]}$ with support in $V$ such that $G_{0}=$ id, $G_{t} \mid X^{(m)}=$ $\operatorname{id}_{X^{(m)}}$ and $G_{1}\left(h_{1}\left(\bar{V}_{3}\right)\right) \subset V_{3}$.

Let $F_{t}$ be the composition of $G_{t}$ and $h_{t}: F_{t}=G_{t} \circ h_{t}$. Then $F_{1}\left(\overline{V_{3}}\right) \subset V_{3}$. For $U=V_{3} \backslash F_{1}\left(\bar{V}_{3}\right),\left(F_{1}\right)^{\ell}(U)(\ell \in \mathbb{Z})$ are disjoint.

We give the proof of Theorem 1.1 (1).

Proof of Theorem 1.1 (1). For the manifold $M^{n}$, we take the $m$-dimensional stratified set $X^{(m)}(2 m<n)$ given in Lemma 2.5. Let $f \in \operatorname{Diff}_{c}^{r}\left(M^{n}\right)_{0}(r \neq n+1)$. By the result of Herman, Mather and Thurston ([11], [14], [16], [23], [2]), $f$ can be written as a product of commutators.

$$
f=\left[a_{1}, b_{1}\right] \cdots\left[a_{k}, b_{k}\right], \quad a_{1}, b_{1}, \ldots, a_{k}, b_{k} \in \operatorname{Diff}_{c}^{r}\left(M^{n}\right)_{0},
$$

where $\left[a_{i}, b_{i}\right]=a_{i} b_{i} a_{i}{ }^{-1} b_{i}{ }^{-1}$. Let $C$ be a compact subset of $M^{n}$ such that the supports of $a_{i}, b_{i}$ as well as the supports of the isotopies $\left\{a_{i t}\right\}_{t \in[0,1]}\left(a_{i 0}=\mathrm{id}\right.$ and $\left.a_{i 1}=a_{i}\right),\left\{b_{i t}\right\}_{t \in[0,1]}\left(b_{i 0}=\mathrm{id}\right.$ and $\left.b_{i 1}=b_{i}\right)$ are contained in $C$.

By using the flow lines of the gradient flow $\Psi_{t}$, we have an isotopy $\left\{G_{t}^{\prime}\right\}_{t \in[0,1]}$ with compact support such that $G_{1}^{\prime}(C) \subset U$, where $U$ is the open neighborhood taken in Lemma 2.5. Then by Lemma 2.5, for $F_{t}$ in Lemma 2.5 and $g=\left(G_{1}^{\prime}\right)^{-1} \circ F_{1} \circ G_{1}^{\prime}$, $g^{\ell}\left(\left(G_{1}^{\prime}\right)^{-1}(U)\right)(\ell \in \mathbb{Z})$ are disjoint.

Put

$$
H=\prod_{i=1}^{k} g^{k-i}\left(\left[a_{1}, b_{1}\right] \cdots\left[a_{i}, b_{i}\right]\right) g^{i-k} .
$$


Then $H$ is an element of $\operatorname{Diff}_{c}^{r}\left(M^{n}\right)_{0}$. Now the conjugate of $H$ by $g$ is as follows:

$$
\begin{aligned}
g H g^{-1} & =\prod_{i=1}^{k} g^{k-i+1}\left(\left[a_{1}, b_{1}\right] \cdots\left[a_{i}, b_{i}\right]\right) g^{i-k-1} \\
& =\prod_{i=0}^{k-1} g^{k-i}\left(\left[a_{1}, b_{1}\right] \cdots\left[a_{i+1}, b_{i+1}\right]\right) g^{i-k} .
\end{aligned}
$$

Hence

$$
\begin{aligned}
H^{-1} g H g^{-1} & =\left(\left[a_{1}, b_{1}\right] \cdots\left[a_{k}, b_{k}\right]\right)^{-1} \prod_{i=0}^{k-1} g^{k-i}\left[a_{i+1}, b_{i+1}\right] g^{i-k} \\
& =f^{-1} \prod_{i=0}^{k-1} g^{k-i}\left[a_{i+1}, b_{i+1}\right] g^{i-k} \\
& =f^{-1}\left[\prod_{i=0}^{k-1} g^{k-i} a_{i+1} g^{i-k}, \prod_{i=0}^{k-1} g^{k-i} b_{i+1} g^{i-k}\right] .
\end{aligned}
$$

Put

$$
A=\prod_{i=0}^{k-1} g^{k-i} a_{i+1} g^{i-k} \quad \text { and } \quad B=\prod_{i=0}^{k-1} g^{k-i} b_{i+1} g^{i-k},
$$

then $A$ and $B$ are elements of $\operatorname{Diff}_{c}^{r}\left(\mathbb{R}^{n}\right)_{0}$. Thus $f$ can be written as a product of two commutators: $f=[A, B]\left[g, H^{-1}\right]$.

Proof of Theorem 1.1 (2). For an even-dimensional compact manifold $M^{2 m}$ which has a handle decomposition without handles of the middle index $m$, Theorem 2.2 together with Theorem 1.1 (1) implies Theorem 1.1 (2) (see [30]).

For the decomposition of an isotopy on an odd dimensional manifold, we used the following lemma (see [30], Remark 4.4).

Lemma 2.6. In Lemma 2.1, let $K^{k}=K^{(k)} \supset K^{(k-1)} \supset \cdots \supset K^{(1)} \supset K^{(0)}$ and $L^{\ell}=L^{(\ell)} \supset L^{(\ell-1)} \supset \cdots \supset L^{(1)} \supset L^{(0)}$ be the stratifications. Then there is an isotopy $\left\{\Phi_{t}: M^{n} \rightarrow M^{n}\right\}_{t \in[0,1]}\left(\Phi_{0}=\mathrm{id}\right)$ with support in a neighborhood of $K^{k}$ such that $\Phi_{1}\left(K^{(a)}\right) \cap f\left(L^{(b)}\right)=\emptyset$ for $a+b+1=n$, and the intersection $\Phi_{1}\left(K^{(a)}\right) \cap f\left(L^{(b)}\right)$ consists of finitely many transverse intersection points for $a+b=n$.

Proof. We proceed as in the proof of Lemma 2.1. Assume that for $u-1 \leq k-1$, there is an isotopy $\left\{\widehat{\Phi}_{t}^{u-1}\right\}_{t \in[0,1]}\left(\widehat{\Phi}_{0}^{u-1}=\right.$ id) such that $\widehat{\Phi}_{1}^{u-1}\left(K^{(a)}\right) \cap f\left(L^{(b)}\right)=\emptyset$ for $a+b+1=n$ and $a \leq u-1$, and the intersection $\widehat{\Phi}_{1}^{u-1}\left(K^{(a)}\right) \cap f\left(L^{(b)}\right)$ consists 
of finitely many transverse points for $a+b=n$ and $a \leq u-1$. Then there is a neighborhood $U_{u-1}$ of $K^{(u-1)}$ such that $\widehat{\Phi}_{1}^{u-1}\left(U_{u-1}\right) \cap \bar{f}\left(L^{(n-u)}\right)=\emptyset$. We cover $K^{(u)} \backslash U_{u-1}$ by finitely many coordinate neighborhoods $\left\{\left(D^{u} \times D^{n-u}\right)_{i}\right\}_{i=1}^{k_{u}}$ such that

$$
\left(K^{(u)} \backslash U_{u-1}\right) \cap\left(D^{u} \times D^{n-u}\right)_{i} \subset\left(D^{u} \times\{0\}\right)_{i}
$$

and $\left\{\left(\operatorname{int}\left(D_{1 / 2}^{u}\right) \times \operatorname{int}\left(D_{1 / 2}^{n-u}\right)\right)_{i}\right\}_{i=1}^{k_{u}}$ still covers $K^{(u)} \backslash U_{u-1}$.

By the proof of Lemma 2.1, we have isotopies $\left\{\Phi_{t}^{u, i}\right\}_{t \in[0,1]}\left(\Phi_{0}^{u, i}=\mathrm{id}, i=1\right.$, $\ldots, k_{u}$ ) with support in $\bigcup_{j=1}^{i}\left(D^{u} \times D^{n-u}\right)_{j}$ such that

$$
K^{(u)} \cap\left(\hat{\Phi}_{1}^{u-1} \circ \Phi_{1}^{u, i}\right)^{-1}\left(f\left(L^{(n-u-1)}\right)\right) \subset \bigcup_{j=i+1}^{k_{u}}\left(\operatorname{int}\left(D_{1 / 2}^{u}\right) \times \operatorname{int}\left(D_{1 / 2}^{n-u}\right)\right)_{j},
$$

and for $\Phi_{t}^{u}=\widehat{\Phi}_{t}^{u-1} \circ \Phi_{t}^{u, k_{u}}, \Phi_{1}^{u}\left(K^{(u)}\right) \cap f\left(L^{(n-u-1)}\right)=\emptyset$.

We modify $\Phi_{t}^{u}$ to obtain $\widehat{\Phi}_{t}^{u}$ such that $\widehat{\Phi}_{1}^{u}\left(K^{(u)}\right) \cap f\left(L^{(n-u)}\right)$ consists of finitely many transverse intersection points.

Since $\Phi_{1}^{u}\left(K^{(u)}\right) \cap f\left(L^{(n-u-1)}\right)=\emptyset,\left(f \mid L^{(n-u)}\right)^{-1}\left(\Phi_{1}^{u}\left(K^{(u)}\right)\right)$ is a closed subset hence is a compact subset in $L^{(n-u)}$. Thus it is compact subset in $L^{(n-u)} \backslash L^{(n-u-1)}$.

Now assume that, for $i \leq k_{u}$, we have an isotopy $\left\{\widehat{\Phi}_{t}^{u, i-1}\right\}_{t \in[0,1]}\left(\widehat{\Phi}_{0}^{u, i-1}=\mathrm{id}\right)$ with support in $\bigcup_{j=1}^{i-1}\left(D^{u} \times D^{n-u}\right)_{j}$ such that

$$
K^{(u)} \cap\left(\Phi_{1}^{u} \circ \widehat{\Phi}_{1}^{u, i-1}\right)^{-1}\left(f\left(L^{(n-u)}\right)\right) \cap \bigcup_{j=1}^{i-1}\left(\operatorname{int}\left(D_{1 / 2}^{u}\right) \times \operatorname{int}\left(D_{1 / 2}^{n-u}\right)\right)_{j} .
$$

consists of transverse intersection points. Then for

$$
L_{i-1}^{\prime n-u}=\left(\Phi_{1}^{u} \circ \widehat{\Phi}_{1}^{u, i-1}\right)^{-1}\left(f\left(L^{(n-u)}\right)\right),
$$

we look at $p_{i}\left(L_{i-1}^{\prime n-u} \cap\left(D^{u} \times D^{n-u}\right)_{i}\right)$ in $D^{n-u}$. More precisely, we look at the map

$$
\begin{aligned}
& p_{i} \circ\left(\Phi_{1}^{u} \circ \widehat{\Phi}_{1}^{u, i-1}\right)^{-1} \circ f: \\
& \quad\left(L^{(n-u)} \backslash L^{(n-u-1)}\right) \cap f^{-1}\left(\left(\Phi_{1}^{u} \circ \widehat{\Phi}_{1}^{u, i-1}\right)\left(\left(\operatorname{int}\left(D^{u}\right) \times \operatorname{int}\left(D^{n-u}\right)\right)_{i}\right)\right) \longrightarrow D^{n-u} .
\end{aligned}
$$

Then by the Sard theorem for $C^{r}$ mappings between the manifolds of the same dimension $(r \geq 1)$, the critical value of $p_{i} \circ\left(\Phi_{1}^{u} \circ \widehat{\Phi}_{1}^{u, i-1}\right)^{-1} \circ f$ is measure zero in $D^{n-u}$. We choose a regular value $q_{i}^{\prime}$ close to 0 .

Let $\left\{\widehat{\Phi}_{t}^{\prime u, i}\right\}_{t \in[0,1]}$ be the isotopy with support in $\left(\operatorname{int}\left(D^{u}\right) \times \operatorname{int}\left(D^{n-u}\right)\right)_{i}$ such that $\widehat{\Phi}_{t}^{\prime u, i}(x, 0)=\left(x, t \mu(x) q_{i}^{\prime}\right)\left(\hat{\Phi}_{0}^{\prime u, i}=\mathrm{id}\right)$. Then since $q_{i}^{\prime}$ is a regular value,

$$
L_{i-1}^{\prime n-u} \cap \widehat{\Phi}_{1}^{\prime u, i}\left(K^{(u)}\right) \cap\left(\operatorname{int}\left(D_{1 / 2}^{u}\right) \times \operatorname{int}\left(D_{1 / 2}^{n-u}\right)\right)_{i}
$$


or

$$
\left(\widehat{\Phi}_{1}^{\prime u, i}\right)^{-1} L_{i-1}^{\prime n-u} \cap K^{(u)} \cap\left(\operatorname{int}\left(D_{1 / 2}^{u}\right) \times \operatorname{int}\left(D_{1 / 2}^{n-u}\right)\right)_{i}
$$

consists of transverse intersection points. Since $q_{i}^{\prime}$ is close to zero, the transversality in $\bigcup_{j=1}^{i-1}\left(\operatorname{int}\left(D_{1 / 2}^{u}\right) \times \operatorname{int}\left(D_{1 / 2}^{n-u}\right)\right)$ is preserved. Hence for $\left\{\widehat{\Phi}_{t}^{u, i}=\widehat{\Phi}_{t}^{u, i-1}\right.$ 。 $\left.\widehat{\Phi}_{t}^{\prime u, i}\right\}_{t \in[0,1]}$,

$$
K^{(u)} \cap\left(\Phi_{1}^{u-1} \circ \widehat{\Phi}_{t}^{u, i}\right)^{-1}\left(f\left(L^{(n-u-1)}\right)\right)=\emptyset
$$

and

$$
K^{(u)} \cap\left(\Phi_{1}^{u-1} \circ \widehat{\Phi}_{t}^{u, i}\right)^{-1}\left(f\left(L^{(n-u)}\right)\right) \cap \bigcup_{j=1}^{i}\left(\operatorname{int}\left(D_{1 / 2}^{u}\right) \times \operatorname{int}\left(D_{1 / 2}^{n-u}\right)\right)_{j}
$$

consists of transverse intersection points.

Then for $\widehat{\Phi}_{t}^{u}=\Phi_{1}^{u-1} \circ \widehat{\Phi}_{t}^{u, k_{u}}$,

$$
K^{(u)} \cap\left(\widehat{\Phi}_{t}^{u}\right)^{-1}\left(f\left(L^{(n-u-1)}\right)\right)=\emptyset
$$

and

$$
K^{(u)} \cap\left(\widehat{\Phi}_{t}^{u}\right)^{-1}\left(f\left(L^{(n-u)}\right)\right)
$$

consists of transverse intersection points. Since $K^{(u)} \cap\left(\widehat{\Phi}_{t}^{u}\right)^{-1}\left(f\left(L^{(n-u)}\right)\right)$ is compact, this is a finite set.

Put $\Phi_{t}=\widehat{\Phi}_{t}^{k}$. Then $\Phi_{t}$ is the desired isotopy.

In the rest of this section, we sketch the proof of Theorem 1.1 (3). We need three more lemmas whose proofs are omitted because they are either straightforward or given by rewriting those in [30].

By using Lemma 2.6 and the argument of the proof of Theorem 2.2, we obtain the following lemma.

Lemma 2.7 ([30], Lemma 6.3). Let $M^{n}$ be a compact $n$-dimensional manifold. Let $P^{p}$ and $Q^{q}$ be $p$-dimensional and $q$-dimensional stratified subsets of $M^{n}$, respectively. Assume that $p+q+1=n$ and that $P^{p} \cap Q^{q}=\emptyset$. Let $P^{p}=P^{(p)} \supset$ $P^{(p-1)} \supset \cdots \supset P^{(0)}$ and $Q^{q}=Q^{(q)} \supset Q^{(q-1)} \supset \cdots \supset Q^{(0)}$ be the stratifications. Then any element $f \in \operatorname{Diff}^{r}\left(M^{n}\right)_{0}$ can be written as a product $f=g \circ h$ such that $g \in \operatorname{Diff}_{c}^{r}\left(M^{n} \backslash k\left(Q^{q}\right)\right)_{0}$ and $h \in \operatorname{Diff}_{c}^{r}\left(M^{n} \backslash P^{(p-1)}\right)_{0}$, where $k \in \operatorname{Diff}_{c}^{r}\left(M^{n} \backslash P^{p}\right)_{0}$ is a diffeomorphism of $M^{n}$ with support in a small neighborhood of $Q^{q}$. Moreover there is an isotopy $\left\{h_{t}\right\}_{t \in[0,1]}$ such that $h_{0}=\mathrm{id}, h_{1}=h, h_{t}$ is the identity on a neighborhood of $P^{(p-1)}$, and for $H(t, x)=h_{t}(x), H\left([0,1] \times P^{p}\right) \cap k\left(Q^{(q-1)}\right)=\emptyset$ and $H\left([0,1] \times\left(P^{p} \backslash P^{(p-1)}\right)\right) \cap k\left(Q^{q} \backslash Q^{(q-1)}\right)$ consists of finitely many transverse intersection points. 
For an odd dimensional compact manifold $M^{2 m+1}$, we considered a handle decomposition of $M^{2 m+1}$ in [30]. Let $M^{2 m+1}=P^{(2 m+1)} \supset \cdots \supset P^{(0)}$ be the stratification by the stable manifolds for the gradient flow for the corresponding Morse function, and $M^{2 m+1}=Q^{(2 m+1)} \supset \cdots \supset Q^{(0)}$ be the stratification by the unstable manifolds for the gradient flow. We look at the stratified subsets $P^{m}=P^{(m)}$ and $Q^{m}=Q^{(m)}$, and we have the following lemma.

Lemma 2.8 ([30], Lemma 6.4). Let $\left\{h_{t}\right\}_{t \in[0,1]}\left(h_{0}=\mathrm{id}\right)$ be a $C^{r}$ isotopy which is the identity on a neighborhood of $P^{(m-1)}$ and $H\left([0,1] \times P^{m}\right) \cap k\left(Q^{(m-1)}\right)=\emptyset$ for $H(t, x)=h_{t}(x)$. Let $V^{m} \subset P^{m}$ be the complement of a neighborhood of $P^{(m-1)}$ where $h_{t}=\mathrm{id}$. Then there is a $C^{\infty}$ isotopy $\left\{\bar{h}_{t}\right\}_{t \in[0,1]}\left(\bar{h}_{0}=\mathrm{id}\right)$ fixing $a$ neighborhood of $P^{(m-1)}$ such that its trace $\bar{H}:[0,1] \times M^{2 m+1} \rightarrow M^{2 m+1}$ is $C^{r}$ close to $H:[0,1] \times M^{2 m+1} \rightarrow M^{2 m+1}$ and $\bar{H} \mid[0,1] \times V^{m}$ is an immersion outside of a finite subset. Moreover the image

$$
\bar{H}\left([0,1] \times V^{m}\right) \subset M^{2 m+1} \backslash\left(P^{(m-1)} \cup k\left(Q^{(m-1)}\right)\right)
$$

has finitely many double point curves which is in general position with respect to the curves $\bar{H}([0,1] \times\{v\})\left(v \in V^{m}\right)$. If $m \geq 2$ these double point curves are disjoint, and if $m=1$, there are at most finitely many triple points and cusps.

Then, using the idea of Burago, Ivanov and Polterovich ([4]), we constructed an isotopy $\left\{a_{t}\right\}_{t \in[0,1]}\left(a_{0}=\mathrm{id}\right)$ with support in a union of disjointly embedded $(2 m+1)$ dimensional open balls embedded in $M^{2 m+1}$ such that $\left(a_{t} \circ \bar{h}_{t}\right)\left(P^{m}\right) \cap k\left(Q^{m}\right)=\emptyset$ $(t \in[0,1])$, and we showed the following lemma.

Lemma 2.9 ([30], Lemma 6.5). For the generic diffeomorphism

$$
\bar{h}=\bar{h}_{1} \in \operatorname{Diff}_{c}^{\infty}\left(M^{2 m+1} \backslash P^{(m-1)}\right)_{0}
$$

given by Lemma 2.8, $\bar{h}$ can be decomposed as $\bar{h}=a \circ \bar{g} \circ \bar{h}^{\prime}$, where $a \in \operatorname{Diff}_{c}^{\infty}\left(\bigsqcup_{i} U_{i}\right)_{0}$, $\bigsqcup_{i} U_{i}$ is a union of $(2 m+1)$-dimensional open balls $U_{i}$ disjointly embedded in $M^{2 m+1}, \bar{g} \in \operatorname{Diff}_{c}^{\infty}\left(M^{2 m+1} \backslash k\left(Q^{m}\right)\right)_{0}$ and $\bar{h}^{\prime} \in \operatorname{Diff}_{c}^{\infty}\left(M^{2 m+1} \backslash P^{m}\right)_{0}$.

Proof of Theorem 1.1 (3). Note that the element $\bar{h}^{-1} \circ h \in \operatorname{Diff}^{r}\left(M^{2 m+1}\right)_{0}$ is close to the identity and it can be decomposed as $\bar{h}^{-1} \circ h=\hat{h} \circ \hat{g}$ with $\hat{h} \in \operatorname{Diff}_{c}^{r}\left(M^{2 m+1} \backslash\right.$ $\left.P^{m}\right)_{0}$ and $\hat{g} \in \operatorname{Diff}_{c}^{r}\left(M^{2 m+1} \backslash k\left(Q^{m}\right)\right)_{0}$ (Remark 5.4 in [30], see Remark 2.10). Then by Lemmas 2.7 and 2.9,

$$
\begin{aligned}
f & =g \circ h=g \circ \bar{h} \circ\left(\bar{h}^{-1} \circ h\right) \\
& =g \circ a \circ \bar{g} \circ \bar{h}^{\prime} \circ \hat{h} \circ \hat{g} \\
& =\left(g \circ a \circ g^{-1}\right) \circ(g \circ \bar{g} \circ \hat{g}) \circ\left(\hat{g}^{-1} \circ \bar{h}^{\prime} \circ \hat{h} \circ \hat{g}\right)
\end{aligned}
$$


and $g \circ a \circ g^{-1} \in \operatorname{Diff}_{c}^{r}\left(g\left(\bigsqcup_{i} U_{i}\right)\right)_{0}, g \circ \bar{g} \circ \hat{g} \in \operatorname{Diff}_{c}^{r}\left(M^{2 m+1} \backslash k\left(Q^{m}\right)\right)_{0}$ and $\hat{g}^{-1} \circ \bar{h}^{\prime} \circ \hat{h} \circ \hat{g} \in \operatorname{Diff}_{c}^{r}\left(M^{2 m+1} \backslash \hat{g}^{-1}\left(P^{m}\right)\right)_{0}$. Noticing that $a$ can be taken as a commutator with support in $\bigsqcup_{i} U_{i}$, Theorem 1.1 (1) implies Theorem 1.1 (3) (see [30]).

It is worth noticing again that, for any compact manifold $M^{n}$, there is a neighborhood of the identity of $\operatorname{Diff}^{r}\left(M^{n}\right)_{0}(1 \leq r \leq \infty, r \neq n+1)$ whose element can be written as a product of four or six commutators([30], Remark 5.4).

Remark 2.10. For a compact manifold $M$, we have a self-indexing Morse function $F: M^{n} \rightarrow[0, n]$. By choosing a Riemannian metric on $M^{n}$, we have the stratification $\left\{X^{(k)}\right\}_{k=0}^{n}$ by the stable manifolds for the gradient flow of the Morse function $F$, and the stratification $\left\{X^{*(n-k)}\right\}_{k=0}^{n}$ by the unstable manifolds. For a compact odd-dimensional manifold $M^{2 m+1}, M^{2 m+1}$ is covered by two open sets $U_{1}=F^{-1}([0, m+2 / 3))$ and $U_{2}=F^{-1}((m+1 / 3,2 m+1])$, where any neighborhood of $X^{(m)} \subset U_{1}$ contains a deformation retract of $U_{1}$ and any neighborhood of $X^{*(m)} \subset U_{2}$ contains a deformation retract of $U_{2}$. Then by the fragmentation lemma ([2]), there is a neighborhood $\mathcal{N}$ of the identity in $\operatorname{Diff}^{r}\left(M^{2 m+1}\right)_{0}$ such that any element $f$ of $\mathcal{N}$ can be written as a product $f=g \circ h$, where $g \in \operatorname{Diff}_{c}^{r}\left(U_{1}\right)_{0}$ and $h \in \operatorname{Diff}_{c}^{r}\left(U_{2}\right)_{0}$. Hence by Theorem 1.1(1), any element $f$ of $\mathcal{N}$ can be written as a product of four commutators of elements of $\operatorname{Diff}^{r}\left(M^{2 m+1}\right)_{0}(1 \leq r \leq \infty$, $r \neq 2 m+2$ ). For a compact even-dimensional manifold $M^{2 m}, M^{2 m}$ is covered by three open sets $U_{1}, U_{2}$ and $U_{3}$. Here, $U_{3}$ is a union of disjointly embedded open balls which is a neighborhood of the set of critical points of index $m$. Let $V_{3}$ be a smaller neighborhood of the critical points of index $m$ such that $\bar{V}_{3} \subset U_{3}$. Then we can put $U_{1}=\left(M^{2 m} \backslash \bar{V}_{3}\right) \cap F^{-1}([0, m+\varepsilon))$ and $U_{2}=\left(M^{2 m} \backslash \bar{V}_{3}\right) \cap F^{-1}((m-\varepsilon, 2 m])$ for a small positive real number $\varepsilon$. Here, we can choose $V_{3}$ so that any neighborhood of $X^{(m-1)} \subset U_{1}$ contains a deformation retract of $U_{1}$ and any neighborhood of $X^{\prime(m-1)} \subset U_{2}$ contains a deformation retract of $U_{2}$. Then by the fragmentation lemma, there is a neighborhood $\mathcal{N}$ of the identity in $\operatorname{Diff}^{r}\left(M^{2 m}\right)_{0}$ such that any element $f$ of $\mathcal{N}$ can be written as a product $f=a \circ g \circ h$, where $g \in \operatorname{Diff}_{c}^{r}\left(U_{1}\right)_{0}$, $h \in \operatorname{Diff}_{c}^{r}\left(U_{2}\right)_{0}$ and $a \in \operatorname{Diff}_{c}^{r}\left(U_{3}\right)_{0}$. Hence by Theorem 1.1(1), any element $f$ of $\mathcal{N}$ can be written as a product of six commutators of elements of $\operatorname{Diff}^{r}\left(M^{2 m}\right)_{0}$ $(1 \leq r \leq \infty, r \neq 2 m+1)$.

\section{Proof of the main theorem}

For an even dimensional compact manifold $M^{2 m}$, we proceed as follows to prove Theorem 1.2. (The proofs of lemmas are given in the next section.)

For the manifold $M^{2 m}$, we consider any smooth triangulation $P$ of it (for the existence of smooth triangulations, see [33], [37], [20], [6]). Let $P^{(k)}$ denote the 
$k$-dimensional skeleton of $P$. Then the $(m-1)$-dimensional skeleton $P^{(m-1)}$ of the triangulation $P$ has the following property:

For each $m$-dimensional simplex $\sigma^{m}$ of $P^{(m)}$, let $\left(P^{(m-1)} \cup \sigma^{m}\right) / \sigma^{m}$ denote the $(m-1)$-dimensional cell complex obtained from $P^{(m-1)} \cup \sigma^{m}$ by identifying $\sigma^{m}$ to a point. Then there is an embedding $\iota$ of $\left(P^{(m-1)} \cup \sigma^{m}\right) / \sigma^{m}$ in $M^{2 m}$ such that, for any neighborhood $U$ of $\iota\left(\left(P^{(m-1)} \cup \sigma^{m}\right) / \sigma^{m}\right)$, there is a diffeomorphism of $M^{2 m}$ isotopic to the identity which maps $P^{(m-1)} \cup \sigma^{m}$ into $U$.

For any smooth triangulation $P$ of $M^{2 m}$, there are a Morse function on $M^{2 m}$ and a Riemannian metric on $M^{2 m}$ such that the stratification by the stable manifolds of the gradient flow is homeomorphic to $P$. Here, in a neighborhood of the barycenter $b_{\sigma^{k}}$ of the simplex $\sigma^{k}$, we can take a coordinate neighborhood $\left(U,\left(x_{1}, \ldots, x_{n}\right)\right)$ such that $\sigma^{k}$ is locally given as $x_{k+1}=\cdots=x_{n}=0$, and the Morse function in a neighborhood of $b_{\sigma^{k}}$ is given by $k-x_{1}{ }^{2}-\cdots-x_{k}{ }^{2}+x_{k+1}{ }^{2}+\cdots+x_{n}{ }^{2}$. The homeomorphism can be constructed so that it sends the stable manifold of the barycenter $b_{\sigma^{k}}$ differentiably to the interior of the simplex $\sigma^{k}$. Moreover the homeomorphism can be constructed so that it sends the stratification $Q$ by the unstable manifolds of the gradient flow to the cell decomposition $P^{*}$ dual to $P$. We show this fact in Section 6 (Proposition 6.1). In this section, we identify the stratification by the stable manifolds with the triangulation $P$ by the homeomorphism and it is denoted by $P$, and then, we call the stratification $Q$ by the unstable manifolds the cell stratification dual to $P$. We call the stable manifolds of $P$ simplices and the unstable manifold of $Q$ dual cells.

Remark 3.1. We may use a cellular complex associated with a handle decomposition of $M^{2 m}$ if it has the above property for each $m$-dimensional cell $\sigma^{m}$. The number $N$ of the $m$-dimensional cells of such a cellular decomposition of $M^{2 m}$ appears in the estimate of the bound for the number of commutators at the end of the proof of Theorem 1.2. We discuss the relationship between the handle decomposition and the cellular decomposition in Section 6 (Proposition 6.2).

Now we look at the $m$-dimensional skeletons $P^{m}$ and $Q^{m}$ of the triangulation $P$ and its dual cell stratification $Q$. These $P^{m}$ and $Q^{m}$ intersect transversely at the barycenters of $m$-dimensional simplices of $P$. Then by an isotopy $f_{t}(t \in[0,1])$, the intersection $f_{t}\left(P^{m}\right) \cap Q^{m}$ becomes very complicated. However, we can treat it as follows.

For the manifold $M^{2 m}$, the statement of Lemma 2.7 is written as follows.

Lemma 3.2. Let $P^{m}$ denote the $m$-dimensional skeleton of a triangulation of a $(2 m)$ dimensional manifold $M^{2 m}$, and $Q^{m}$, the m-dimensional skeleton of the dual cell stratification. Let $P^{(i)}$ and $Q^{(i)}$ denote the $i$-dimensional skeletons $(i=m-2$, $m-1)$ of $P^{m}$ and $Q^{m}$, respectively. Then any element $f \in \operatorname{Diff}^{r}\left(M^{2 m}\right)_{0}$ can be written as a product $f=g \circ h$ such that $g \in \operatorname{Diff}_{c}^{r}\left(M^{2 m} \backslash k\left(Q^{m}\right)\right)_{0}$ and $h \in \operatorname{Diff}_{c}^{r}\left(M^{2 m} \backslash P^{(m-2)}\right)_{0}$, where $k \in \operatorname{Diff}_{c}^{r}\left(M^{2 m} \backslash P^{m}\right)_{0}$ is a diffeomorphism 
of $M^{n}$ with support in a small neighborhood of $Q^{m}$. Moreover there is an isotopy $\left\{h_{t}\right\}_{t \in[0,1]}$ which has the following properties:

(1) $h_{0}=\mathrm{id}, h_{1}=h$, and $h_{t}$ is the identity on a neighborhood of $P^{(m-2)}$.

(2) $\operatorname{For} H(t, x)=h_{t}(x)$,

$H\left([0,1] \times P^{(m-1)}\right) \cap k\left(Q^{(m-1)}\right)=\emptyset$ and $H\left([0,1] \times P^{m}\right) \cap k\left(Q^{(m-2)}\right)=\emptyset$.

(3) For each $(m-1)$-dimensional simplex $\sigma^{m-1}$ of $P^{(m-1)}$ and each $m$-dimensional cell $\tau^{m}$ of $Q^{m}$, the intersection $H\left([0,1] \times \sigma^{m-1}\right) \cap k\left(\tau^{m}\right)$ is transverse. Thus $H\left([0,1] \times P^{(m-1)}\right) \cap k\left(Q^{m}\right)$ is a finite set.

Then, if $2 m \geq 4$, we can separate the image $H\left([0,1] \times P^{(m-1)}\right)$ from $k\left(Q^{m}\right)$ by an argument similar to the proof of Lemmas 2.8 and 2.9.

First, we approximate the isotopy $H$ by a generic one, say $\bar{H}$. Let

$$
\left\{\bar{h}_{t}\right\}_{t \in[0,1]} \subset \operatorname{Diff}_{c}^{\infty}\left(M^{2 m} \backslash P^{(m-2)}\right) \quad\left(\bar{h}_{0}=\mathrm{id}\right)
$$

be a $C^{\infty}$ approximation of $\left\{h_{t}\right\}_{t \in[0,1]} \subset \operatorname{Diff}_{c}^{r}\left(M^{2 m} \backslash P^{(m-2)}\right)$ generic with respect to $P^{m}$ and $k\left(Q^{m}\right)$ such that $\bar{h}_{t}$ is the identity on a neighborhood of $P^{(m-2)}$. Then $\bar{H}(t, x)=\bar{h}_{t}(x)$ has the following properties:

(0) $\bar{H}:[0,1] \times M^{2 m} \rightarrow M^{2 m}$ is close to $H:[0,1] \times M^{2 m} \rightarrow M^{2 m}$ and $\bar{h}_{t}$ is the identity on a neighborhood of $P^{(m-2)}$.

(1) The restriction

$$
\bar{H} \mid\left([0,1] \times V^{m-1}\right):[0,1] \times V^{m-1} \longrightarrow M^{2 m}
$$

is an immersion, where $V^{m-1}\left(\subset P^{(m-1)}\right)$ is the complement of a neighborhood of $P^{(m-2)} \subset P^{(m-1)}$ where $\bar{h}_{t}$ is the identity.

(2) $\bar{H}\left([0,1] \times P^{(m-1)}\right) \cap k\left(Q^{(m-1)}\right)=\emptyset$ and $\bar{H}\left([0,1] \times P^{m}\right) \cap k\left(Q^{(m-2)}\right)=\emptyset$.

(3) $\bar{H}\left([0,1] \times P^{(m-1)}\right) \cap k\left(Q^{m}\right)$ is a finite set:

$$
\bar{H}\left([0,1] \times P^{(m-1)}\right) \cap k\left(Q^{m}\right)=\left\{\bar{H}\left(s_{i}, v_{i}\right) \mid i=1, \ldots, r\right\} .
$$

(4) $\bar{H}\left([0,1] \times\left\{v_{i}\right\}\right) \cap k\left(Q^{m}\right)=\bar{H}\left(s_{i}, v_{i}\right)(i=1, \ldots, r)$.

(5) $\bar{H}\left([0,1] \times\left\{v_{i}\right\}\right)$ does not contain double points of $\bar{H}\left([0,1] \times P^{(m-1)}\right)(i=1$, $\ldots, r)$.

(6) $\bar{H} \mid[0,1] \times P^{(m-1)}$ restricted to a neighborhood of $[0,1] \times\left\{v_{i}\right\}$ in $[0,1] \times P^{(m-1)}$ is an embedding $(i=1, \ldots, r)$, and

(7) $\bar{H}\left(\left[s_{i}, 1\right] \times\left\{v_{i}\right\}\right)(i=1, \ldots, r)$ are disjoint. 
Here, the statements (1)-(7) hold for generic $\bar{H}$ (or the properties (1)-(7) are generic in the space of isotopies). In particular, the statement (5) holds because the inverse image of the double point set of $\bar{H}\left([0,1] \times P^{(m-1)}\right)$ is a finite set which is in general position with respect to $[0,1] \times\left\{v_{i}\right\}(i=1, \ldots, r)$ and $2 m \geq 4$.

Note that for the proof of uniform perfectness, we can approximate the diffeomorphism for a bounded number of times. In fact in this case, $f_{1}=g_{1} \circ h_{1}=$ $g_{1} \circ \bar{h}_{1} \circ\left(\bar{h}_{1}^{-1} \circ h_{1}\right)$ and $\bar{h}_{1}^{-1} \circ h_{1} \in \operatorname{Diff}^{r}\left(M^{2 m}\right)$ is close to the identity. By Remark 2.10, $\bar{h}_{1}^{-1} \circ h_{1}$ can be written as a product of six commutators.

For the above disjoint curves $\bar{H}\left(\left[s_{i}, 1\right] \times\left\{v_{i}\right\}\right)$, we can construct isotopies as in Lemma 2.9 which was used to prove Theorem 1.1 (3).

Lemma 3.3. For the above generic isotopy $\left\{\bar{h}_{t}\right\}_{t \in[0,1]}$, there is a neighborhood $U_{i}(i=1, \ldots, r)$ of the curve $\bar{H}\left(\left[s_{i}, 1\right] \times\left\{v_{i}\right\}\right) \subset M^{2 m}$ diffeomorphic to a $(2 m)$ dimensional ball such that $U_{i}$ are disjoint and there is an isotopy $\left\{a_{t}\right\}_{t \in[0,1]}\left(a_{0}=\mathrm{id}\right)$ with support in $\bigsqcup_{i=1}^{r} U_{i}$ such that, for $h_{t}^{\prime}=a_{t} \circ \bar{h}_{t}$,

$$
h_{t}^{\prime}\left(P^{(m-1)}\right) \cap k\left(Q^{m}\right)=\emptyset \quad(t \in[0,1]) .
$$

Note that $a_{t} \in \operatorname{Diff}_{c}^{r}\left(\bigsqcup_{i=1}^{r} U_{i}\right)_{0}$ can be taken as one commutator with support in $\bigsqcup_{i=1}^{r} U_{i}($ see [31]).

Since $h_{t}^{\prime}\left(P^{(m-1)}\right) \cap k\left(Q^{m}\right)=\emptyset(t \in[0,1])$, by Lemma 2.3 , there are isotopies $\left\{g_{t}^{\prime}\right\}_{t \in[0,1]} \subset \operatorname{Diff}_{c}^{r}\left(M^{2 m} \backslash k\left(Q^{m}\right)\right)$ and $\left\{h_{t}^{\prime \prime}\right\}_{t \in[0,1]} \subset \operatorname{Diff}_{c}^{r}\left(M^{2 m} \backslash P^{(m-1)}\right)$ such that $h_{1}^{\prime}=g_{1}^{\prime} \circ h_{1}^{\prime \prime}$. In other words, $g_{t}^{\prime}$ and $h_{t}^{\prime \prime}(t \in[0,1])$ are the identity on neighborhoods of $k\left(Q^{m}\right)$ and $P^{(m-1)}$, respectively. Note that, by taking $h_{t}^{\prime \prime}$ generically on $P^{m}, h_{t}^{\prime \prime}\left(P^{m}\right) \cap k\left(Q^{(m-2)}\right)=\emptyset$.

Put $h_{t}^{(0)}=h_{t}^{\prime \prime}$. Then $h_{t}^{(0)}$ is the identity on a neighborhood of $P^{(m-1)}$ and $h_{t}^{(0)}\left(P^{m}\right) \cap k\left(Q^{(m-2)}\right)=\emptyset(t \in[0,1])$.

We look at the intersection $h_{t}^{(0)}\left(P^{m}\right) \cap k\left(Q^{m}\right)$. At time 0 , the intersection $h_{0}^{(0)}\left(P^{m}\right) \cap k\left(Q^{m}\right)$ is the set of the points near the barycenters of $m$-dimensional simplices. The image under the isotopy $h_{t}^{(0)}$ of an $m$-dimensional simplex $\sigma^{m}$ intersects $k\left(Q^{(m-1)}\right)$ and $k\left(Q^{m}\right)$. We assume $2 m \geq 6$ and we are going to construct an isotopy with support in the union of disjointly embedded balls which removes the intersection of $\sigma^{m}$ and $k\left(Q^{m}\right)$ except on the dual $m$-dimensional cell.

This is the main part of the proof of our Theorem 1.2.

In fact, for an $m$-dimensional simplex $\sigma^{m}$, we can remove the intersection of the image of the isotopy of $\sigma^{m}$ and $k\left(Q^{(m-1)}\right)$ in a way similar to Lemma 3.3, and then we can remove the intersection of the resultant isotopy of $\sigma^{m}$ and $k\left(Q^{m} \backslash \sigma^{m *}\right)$, where $\sigma^{m *}$ is the $m$-dimensional cell of $Q^{m}$ dual to $\sigma^{m}$. For the latter process, we will find the Whitney disks which guide the construction of isotopy to reduce the order of the intersection point set. After removing the intersection of an $m$-dimensional simplex $\sigma^{m}$ and $k\left(Q^{m} \backslash \sigma^{m *}\right)$, we continue the process for other $m$-dimensional simplices.

More precisely, we construct the isotopies inductively, in Lemmas 3.4-3.7. 
Let $\sigma_{i}^{m}(i=1, \ldots, N)$ be the $m$-dimensional simplices of $P^{m}$. For $0 \leq j \leq N$, assume that we have an isotopy

$$
\left\{h_{t}^{(j)}\right\}_{t \in[0,1]} \subset \operatorname{Diff}^{r}\left(M^{2 m}\right)_{0} \quad\left(h_{0}^{(j)}=\mathrm{id}\right)
$$

such that $h_{t}^{(j)}$ is the identity on a neighborhood of $P^{(m-1)} \cup \bigcup_{i=1}^{j} \sigma_{i}^{m}$. Let $\bar{h}_{t}^{(j)}$ be a $C^{\infty}$ approximation of $h_{t}^{(j)}$ generic with respect to $P^{m}$ and $k\left(Q^{m}\right)$ such that $\bar{h}_{t}^{(j)}$ is the identity on a neighborhood of $P^{(m-1)} \cup \bigcup_{i=1}^{j} \sigma_{i}^{m}$. Then $\bar{H}^{(j)}(t, x)=\bar{h}_{t}^{(j)}(x)$ has the following properties:

(0) $\bar{H}^{(j)}:[0,1] \times M^{2 m} \rightarrow M^{2 m}$ is close to $H^{(j)}:[0,1] \times M^{2 m} \rightarrow M^{2 m}$ defined by $H^{(j)}(t, x)=h_{t}^{(j)}(x)$ and $\bar{h}_{t}^{(j)}$ is the identity on a neighborhood of $P^{(m-1)} \cup$ $\bigcup_{i=1}^{j} \sigma_{i}^{m}$.

(1) The restriction

$$
\bar{H}^{(j)} \mid[0,1] \times V_{(j)}^{m}:[0,1] \times V_{(j)}^{m} \longrightarrow M^{2 m}
$$

is an immersion outside of a 1-dimensional subset (a codimension $m$ subset) of $[0,1] \times V_{(j)}^{m}$, where $V_{(j)}^{m}\left(\subset P^{m}\right)$ is the complement of a neighborhood of $P^{(m-1)}$ in $P^{m}$ where $\bar{h}_{t}^{(j)}$ is the identity.

(2) $\bar{H}^{(j)}\left([0,1] \times P^{(m-1)}\right) \cap k\left(Q^{(m-1)}\right)=\emptyset$ and $\bar{H}^{(j)}\left([0,1] \times P^{m}\right) \cap k\left(Q^{(m-2)}\right)=$ $\emptyset$.

(3) $\bar{H}^{(j)}\left([0,1] \times P^{m}\right) \cap k\left(Q^{(m-1)}\right)$ is a finite set:

$$
\bar{H}^{(j)}\left([0,1] \times P^{m}\right) \cap k\left(Q^{(m-1)}\right)=\left\{\bar{H}^{(j)}\left(s_{i}^{(j)}, v_{i}^{(j)}\right) \mid i=1, \ldots, r^{(j)}\right\} .
$$

(4) $\bar{H}^{(j)}\left([0,1] \times\left\{v_{i}^{(j)}\right\}\right) \cap k\left(Q^{(m-1)}\right)=\bar{H}^{(j)}\left(s_{i}^{(j)}, v_{i}^{(j)}\right)\left(i=1, \ldots, r^{(j)}\right)$.

(5) $\bar{H}^{(j)}\left([0,1] \times\left\{v_{i}^{(j)}\right\}\right)$ does not contain double points of $\bar{H}^{(j)}\left([0,1] \times P^{m}\right)(i=1$, $\left.\ldots, r^{(j)}\right)$.

(6) $\bar{H}^{(j)} \mid[0,1] \times P^{m}$ restricted to a neighborhood of $[0,1] \times\left\{v_{i}^{(j)}\right\}$ in $[0,1] \times P^{m}$ is an embedding $\left(i=1, \ldots, r^{(j)}\right)$, and

(7) $\bar{H}^{(j)}\left(\left[s_{i}^{(j)}, 1\right] \times\left\{v_{i}^{(j)}\right\}\right)$ are disjoint.

Here, the statements (1)-(7) hold for generic $\bar{H}^{(j)}$. In particular, for the statement (1), we notice that the set of rank $m$ matrices in the space of $(m+1) \times(2 m)$ matrices is codimension $m$ ([22]). The statement (6) holds because the inverse image of the double point set of $\bar{H}^{(j)}\left([0,1] \times P^{m}\right)$ is 2-dimensional in $[0,1] \times P^{m}$ which is in general position with respect to $[0,1] \times\left\{v_{i}^{(j)}\right\}\left(i=1, \ldots, r^{(j)}\right)$ and $2 m \geq 6$. 
Lemma 3.4. For the above generic isotopy $\left\{\bar{h}_{t}^{(j)}\right\}_{t \in[0,1]}$, there is a neighborhood $U_{i}^{(j)}$ $\left(i=1, \ldots, r^{(j)}\right)$ of the curve $\bar{H}^{(j)}\left(\left[s_{i}^{(j)}, 1\right] \times\left\{v_{i}^{(j)}\right\}\right) \subset M^{2 m}$ diffeomorphic to a $(2 m)$ dimensional ball such that $U_{i}^{(j)}$ are disjoint and there is an isotopy $\left\{a_{t}^{(j+1)}\right\}_{t \in[0,1]}$ $\left(a_{0}^{(j+1)}=\mathrm{id}\right)$ with support in $\bigsqcup_{i=1}^{r^{(j)}} U_{i}^{(j)}$ such that, for ${h^{\prime}}_{t}^{(j)}=a_{t}^{(j+1)} \circ \bar{h}_{t}^{(j)}$,

$$
{h^{\prime}}_{t}^{(j)}\left(P^{m}\right) \cap k\left(Q^{(m-1)}\right)=\emptyset \quad(t \in[0,1]) .
$$

Note again that $a_{t}^{(j+1)} \in \operatorname{Diff}_{c}^{r}\left(\bigsqcup_{i=1}^{r^{(j)}} U_{i}^{(j)}\right)_{0}$ can be taken as one commutator with support in $\bigsqcup_{i=1}^{r^{(j)}} U_{i}^{(j)}$ (see [31]).

The isotopy ${h^{\prime}}_{t}^{(j)}$ given by Lemma 3.4 has the following properties.

(0) $h^{\prime(j)}$ is the identity on a neighborhood of $P^{(m-1)} \cup \bigcup_{i=1}^{j} \sigma_{i}^{m}$.

(1) $H^{\prime(j)}\left([0,1] \times P^{m}\right) \cap k\left(Q^{(m-1)}\right)=\emptyset$.

(2) $h_{t}^{\prime(j)}$ is generic with respect to $P^{m}$ and $k\left(Q^{m}\right)$.

Now we look at the intersection ${h^{\prime}}_{t}^{(j)}\left(P^{m}\right) \cap k\left(Q^{m}\right)$. Since ${h^{\prime}}_{t}^{(j)}$ is the identity on a neighborhood of $P^{(m-1)} \cup \bigcup_{i=1}^{j} \sigma_{i}^{m}$, the intersection ${h^{\prime}}_{t}^{(j)}\left(\sigma_{i}^{m}\right) \cap k\left(Q^{m}\right)$ for $i \leq j$ is always the one point set $\sigma_{i}^{m} \cap k\left(\sigma_{i}^{m *}\right)$, where $\sigma_{i}^{m *}$ is the $m$-dimensional cell of $Q^{m}$ dual to $\sigma_{i}^{m}(i \leq j)$. For the simplex $\sigma_{j+1}^{m}$, the intersection ${h^{\prime}}_{t}^{(j)}\left(\sigma_{j+1}^{m}\right) \cap$ $k\left(Q^{m}\right)$ is a finite set which vary with respect to the parameter $t$. If $2 m \geq 6$, we can find the Whitney disks which guide to reduce the order of intersection point set $h^{\prime(j)}\left(\sigma_{j+1}^{m}\right) \cap k\left(Q^{m} \backslash \sigma_{j+1}^{m *}\right)$, where $\sigma_{j+1}^{m *}$ is the $m$-dimensional cell of $Q^{m}$ dual to $\sigma_{j+1}^{m}$ as we explain now.

For the $m$-dimensional simplex $\sigma_{j+1}^{m}$ of $P^{m}$, the intersection of $\sigma_{j+1}^{m}$ and $k\left(Q^{m}\right)$ is just one point which is the intersection of $\sigma_{j+1}^{m}$ and $k\left(\sigma_{j+1}^{m *}\right)$, Then the behavior of the intersection ${h^{\prime}}_{t}^{(j)}\left(\sigma_{j+1}^{m}\right) \cap k\left(\sigma_{j+1}^{m *}\right)$ it rather complicated. Hence we look at $H^{\prime(j)}\left([0,1] \times \sigma_{j+1}^{m}\right) \cap k\left(Q^{m} \backslash \sigma_{j+1}^{m *}\right)$ or ${h^{\prime}}_{t}^{(j)}\left(\sigma_{j+1}^{m}\right) \cap k\left(Q^{m} \backslash \sigma_{j+1}^{m *}\right)$. First, note that ${h^{\prime}}_{t}^{(j)}\left(\sigma_{j+1}^{m}\right) \cap k\left(Q^{m} \backslash \sigma_{j+1}^{m *}\right)$ is the empty set for small $t$, and since ${h^{\prime}}_{t}^{(j)}\left(\sigma_{j+1}^{m}\right) \cap$ $k\left(Q^{(m-1)}\right)=\emptyset\left(\operatorname{and}{h^{\prime}}_{t}^{(j)}\left(P^{(m-1)}\right) \cap k\left(Q^{m}\right)=\emptyset\right)$, the algebraic intersection number of the two $m$-dimensional cells ${h^{\prime}}_{t}^{(j)}\left(\sigma_{j+1}^{m}\right)$ and $k\left(\tau^{m}\right)(t \in[0,1])$ is always 0 for each $m$-dimensional cell $\tau^{m}$ of the dual cell complex $Q^{m}$ other than $\sigma_{j+1}^{m *}$.

If we look at the movement of the intersection ${h^{\prime}}_{t}^{(j)}\left(\sigma_{j+1}^{m}\right) \cap k\left(\tau^{m}\right)$ with respect to the parameter $t$, there happen a finite number of generations of pairs of intersection points and cancellations of pairs of intersection points. For generic ${H^{\prime}}^{(j)}$ or $h^{\prime(j)}$, the values of the parameters $t$ of generations and cancellations are different. This genericity argument follows from the following well known lemma.

Lemma 3.5. Consider the space of $C^{\infty}$ maps $F: \mathbb{R} \times \mathbb{R}^{m} \rightarrow \mathbb{R}^{m}$. Then, for generic $F$, the inverse image of a generic point $y \in \mathbb{R}^{m}$ consists of regular points and fold 
points for $F_{t}=F(t, \bullet)$. At a fold point $x$ for $F_{t}$, by changing the coordinates of $\mathbb{R}^{m}$ (both of the second factor of $\mathbb{R} \times \mathbb{R}^{m}$ and the target $\mathbb{R}^{m}$ ), $F_{t}$ is locally written as

$$
F_{t}\left(x_{1}, \ldots, x_{m}\right)=\left(x_{1}, \ldots, x_{m-1}, y_{m}\left(t, x_{1}, \ldots, x_{m}\right)\right) \text {, }
$$

where $\frac{\partial y_{m}}{\partial x_{m}}=0, \frac{\partial y_{m}}{\partial t} \neq 0$ and $\frac{\partial^{2} y_{m}}{\partial x_{m}^{2}} \neq 0$ at $x$. The fold points are discrete in $F^{-1}(y)$ and correspond to the generations or cancellations of pairs of intersection points.

We use this Lemma 3.5 in the following way. We take a tubular neighborhood of $k\left(\tau^{m}\right)$ and the projection $p_{k\left(\tau^{m}\right)}$ to the fiber which is an $m$-dimensional disk, and look at the map $p_{k\left(\tau^{m}\right)} \circ\left(H^{\prime(j)} \mid[0,1] \times \sigma_{j+1}^{m}\right)$. Then for generic $H^{\prime(j)}$, by using Lemma 3.5 , there are only finitely many generations and cancellations of pairs of intersections in the family $\left\{h_{t}^{\prime(j)}\left(\sigma_{j+1}^{m}\right) \cap k\left(\tau^{m}\right)\right\}_{t \in[0,1]}$.

We are going to construct the disks associated with the intersection $H^{\prime(j)}([0,1] \times$ $\left.\sigma_{j+1}^{m}\right) \cap k\left(\tau^{m}\right)$ for an $m$-dimensional cell $\tau^{m}$ of $Q^{m}$ other than $\sigma_{j+1}^{m *}$.

For a generation of a pair of intersection points, the intersection points near the generation point are written as $h_{t}^{\prime(j)}\left(x_{t}\right)$ and $h^{\prime}{ }_{t}^{(j)}\left(y_{t}\right)\left(t \in\left[t_{0}, t_{0}+\varepsilon_{0}\right)\right)$, where ${h^{\prime}}_{t_{0}}^{(j)}\left(x_{t_{0}}\right)=h^{\prime}{ }_{t_{0}}{ }^{(j)}\left(y_{t_{0}}\right)$ is the generation point. Here, $x_{t}$ and $y_{t}$ are continuous functions written as $x_{t}=\left(c_{1}, \ldots, c_{m-1}, \sqrt{t-t_{0}}\right)$ and $y_{t}=\left(c_{1}, \ldots, c_{m-1},-\sqrt{t-t_{0}}\right)$, respectively, for a suitable choice of coordinate around $\left(t_{0}, x_{t_{0}}\right)=\left(t_{0}, y_{t_{0}}\right) \in$ $[0,1] \times \sigma_{j+1}^{m}$, where $c_{1}, \ldots, c_{m-1}$ are constants.

We take a flat metric on the $m$-dimensional simplex $\sigma_{j+1}^{m}$ and we draw the geodesic segment $\overline{x_{t} y_{t}}$ in $\sigma_{j+1}^{m}$ joining the intersection points $x_{t}$ and $y_{t}\left(t \in\left[t_{0}, t_{0}+\varepsilon_{0}\right)\right)$.

Once we choose the pair of intersection points to be joined by the geodesic segment, we continue joining them as the parameter $t$ increases unless one of these intersection points meets a cancellation point.

For a cancellation of a pair of intersections, the intersection points near the cancellation point are written as ${h^{\prime}}_{t}^{(j)}\left(x_{t}\right)$ and ${h^{\prime}}_{t}^{(j)}\left(y_{t}\right)\left(t \in\left(t_{0}-\varepsilon_{0}, t_{0}\right]\right)$, where ${h^{\prime}}_{t_{0}}^{(j)}\left(x_{t_{0}}\right)=$ $h^{\prime}{ }_{t_{0}}^{(j)}\left(y_{t_{0}}\right)$ is the cancellation point. Here, $x_{t}$ and $y_{t}$ are continuous functions written as $x_{t}=\left(c_{1}, \ldots, c_{m-1}, \sqrt{-t+t_{0}}\right)$ and $y_{t}=\left(c_{1}, \ldots, c_{m-1},-\sqrt{-t+t_{0}}\right)$, respectively, for a suitable choice of coordinate around $\left(t_{0}, x_{t_{0}}\right)=\left(t_{0}, y_{t_{0}}\right) \in[0,1] \times \sigma_{j+1}^{m}$, where $c_{1}, \ldots, c_{m-1}$ are constants.

Assume that we have chosen geodesic segments for the intersection points such that $t<t_{0}$. Let $x_{t}^{\prime}\left(t \in\left(t_{0}-\varepsilon_{0}, t_{0}\right)\right)$ be the other endpoint of the geodesic segment containing $x_{t}$, and $y_{t}^{\prime}\left(t \in\left(t_{0}-\varepsilon_{0}, t_{0}\right)\right)$ be the other endpoint of the geodesic segment containing $y_{t}$. There are two cases. In the case where $x_{t_{0}}^{\prime} \neq y_{t_{0}}^{\prime}$, that is, if it is a cancellation of intersection points belonging to different geodesic segments $\overline{x_{t} x_{t}^{\prime}}$ and $\overline{y_{t} y_{t}^{\prime}}$ in $\{t\} \times \sigma_{j+1}^{m}\left(t \in\left(t_{0}-\varepsilon_{0}, t_{0}\right)\right)$, we draw the geodesic triangle joining the 3 points $x_{t_{0}}=y_{t_{0}}, x_{t_{0}}^{\prime}$ and $y_{t_{0}}^{\prime}$ in $\left\{t_{0}\right\} \times \sigma_{j+1}^{m}$, and continue to draw the geodesic segment $\overline{x_{t}^{\prime} y_{t}^{\prime}}$ joining $x_{t}^{\prime}$ and $y_{t}^{\prime}$ in $\{t\} \times \sigma_{j+1}^{m}\left(t \in\left(t_{0}, t_{0}+\varepsilon_{0}\right)\right)$. In the case where $x_{t_{0}}^{\prime}=y_{t_{0}}^{\prime}$, that is, if it is a cancellation of intersection points of the same geodesic 
segment $\overline{x_{t} y_{t}}$ in $\{t\} \times \sigma_{j+1}^{m}\left(t \in\left(t_{0}-\varepsilon_{0}, t_{0}\right), x_{t}^{\prime}=y_{t}\right.$ and $\left.y_{t}^{\prime}=x_{t}\right)$, we add the auxiliary band

$$
\bigcup_{t \in\left[t_{0}-\varepsilon, t_{0}\right]}[t, 1] \times\left\{x_{t}\right\} \cup \bigcup_{t \in\left[t_{0}-\varepsilon, t_{0}\right]}[t, 1] \times\left\{y_{t}\right\},
$$

which contains the curve $\left[t_{0}, 1\right] \times\left\{x_{t_{0}}\right\}=\left[t_{0}, 1\right] \times\left\{y_{t_{0}}\right\}$, where $\varepsilon\left(<\varepsilon_{0}\right)$ is a small positive real number. Note that the image of the auxiliary band does not contain double points of $H^{\prime(j)}\left([0,1] \times \sigma_{j+1}^{m}\right)$ for generic $H^{\prime(j)}$, and hence $H^{\prime(j)}$ restricted to the auxiliary band is an embedding into $M^{2 m} \backslash k\left(Q^{(m-1)}\right)$.

Now we have a family of geodesic segments in $\sigma_{j+1}^{m}$ moving with respect to the parameter $t$ and there are only finitely many times $t_{i}\left(i=1, \ldots, \bar{r}^{(j)}\right)$ when there appear geodesic triangles.

We are assuming that $2 m \geq 6$, and for generic $h_{t}^{(j)}$, the family of geodesic segments satisfies the following properties because the preimage of the double points of ${h^{\prime}}_{t}^{(j)}\left(P^{m}\right)$ is 1-dimensional in $[0,1] \times \sigma_{j+1}^{m}$.

(1) The geodesic segments in $\sigma_{j+1}^{m}$ joining the pairs of intersection points in $\left(h_{t}^{\prime(j)}\right)^{-1}\left(k\left(\tau^{m}\right)\right)$ never contain the preimage of double points of $\left(h_{t}^{\prime(j)}\right)\left(P^{m}\right)$.

(2) The geodesic triangles never contain the preimage of double points of $\left(h_{t}^{\prime(j)}\right)\left(P^{m}\right)$.

For $t_{i}\left(i=1, \ldots, \bar{r}^{(j)}\right)$, let $Y$ be the union of the geodesic triangle with the three vertices $x_{t_{i}}=y_{t_{i}}, x_{t_{i}}^{\prime}$ and $y_{t_{i}}^{\prime}$ in $\left\{t_{i}\right\} \times \sigma_{j+1}^{m}$, the geodesic segments $\overline{x_{t} x_{t}^{\prime}}$ and $\overline{y_{t} y_{t}^{\prime}}$ in $\{t\} \times \sigma_{j+1}^{m},\left(t \in\left(t_{i}-\varepsilon_{i}, t_{i}\right)\right)$ and the geodesic segments $\overline{x_{t}^{\prime} y_{t}^{\prime}}$ in $\{t\} \times \sigma_{j+1}^{m}$ $\left(t \in\left(t_{i}, t_{i}+\varepsilon_{i}\right)\right)$ :

$$
\begin{aligned}
Y & =\left(\bigcup_{t \in\left(t_{i}-\varepsilon_{i}, t_{i}\right)}\{t\} \times \overline{x_{t} x_{t}^{\prime}}\right) \cup\left(\bigcup_{t \in\left(t_{i}-\varepsilon_{i}, t_{i}\right)}\{t\} \times \overline{y_{t} y_{t}^{\prime}}\right) \\
& \cup\left(\left\{t_{i}\right\} \times \Delta x_{t_{i}} x_{t_{i}}^{\prime} y_{t_{i}}^{\prime}\right) \cup\left(\bigcup_{t \in\left(t_{i}, t_{i}+\varepsilon_{i}\right)}\{t\} \times \overline{x_{t}^{\prime} y_{t}^{\prime}}\right) \\
& \subset\left(t_{i}-\varepsilon_{i}, t_{i}+\varepsilon_{i}\right) \times \sigma_{j+1}^{m} .
\end{aligned}
$$

We deform it to obtain a 2-dimensional manifold $Y^{\prime}$ embedded in $\left(t_{i}-\varepsilon_{i}, t_{i}+\varepsilon_{i}\right) \times$ $\sigma_{j+1}^{m}$ such that

$$
\begin{gathered}
\partial Y^{\prime}=\partial Y=\left\{\left(t, x_{t}^{\prime}\right)\right\}_{t \in\left(t_{i}-\varepsilon_{i}, t_{i}+\varepsilon_{i}\right)} \cup\left\{\left(t, y_{t}^{\prime}\right)\right\}_{t \in\left(t_{i}-\varepsilon_{i}, t_{i}+\varepsilon_{i}\right)} \\
\cup\left\{\left(t, x_{t}\right)\right\}_{t \in\left(t_{i}-\varepsilon_{i}, t_{i}\right]} \cup\left\{\left(t, y_{t}\right)\right\}_{t \in\left(t_{i}-\varepsilon_{i}, t_{i}\right]} \\
\subset\left(t_{i}-\varepsilon_{i}, t_{i}+\varepsilon_{i}\right) \times \sigma_{j+1}^{m},
\end{gathered}
$$

and $Y^{\prime}$ coincides with $Y$ for $\left|t-t_{i}\right| \geq \varepsilon_{i} / 2$ and the intersection of $Y^{\prime}$ and $\{t\} \times \sigma_{j+1}^{m}$ is a union of two disjoint differentiable curves near the original geodesic segments 
for $t \in\left[t_{i}-\varepsilon_{i} / 2, t_{i}\right)$ and is one differentiable curve near the geodesic triangle for $t \in\left[t_{i}, t_{i}+\varepsilon_{i} / 2\right]$.

Now we look at the union $Z$ of geodesic segments which are not modified by the above operation and the manifolds $Y^{\prime}$ for all $t_{i}\left(i=1, \ldots, \bar{r}^{(j)}\right)$. If there are auxiliary bands we add them to $Z$ and modify it to make $Z$ an embedded 2-dimensional manifold with boundary in $[0,1] \times \sigma_{j+1}^{m}$.

For a generic choice of the isotopy $H^{\prime(j)}$ and manifolds $Y^{\prime}$, if $2 m \geq 8, Z$ is a union of disjointly embedded 2-dimensional disks in $[0,1] \times \sigma_{j+1}^{m}$. If $2 m=6$, the 2dimensional disks may intersect in $[0,1] \times \sigma_{j+1}^{3}$ creating finitely many double points.

For $2 m \geq 8$, the fact that a connected component of the union $Z$ is diffeomorphic to a 2-dimensional disk can be seen as follows: Consider the space obtained from $Z$ by identifying the points in each connected component of $Z \cap\left(\{t\} \times \sigma_{j+1}^{m}\right)$. Then it is a graph with vertices corresponding to the generation points and cancellation points. The generation points correspond to the vertices of valency 1 and the cancellation points correspond to the vertices of valency 3 except the cancellation points with auxiliary bands. For the cancellation points with auxiliary bands, the auxiliary bands become edges ending at $\{1\} \times \sigma_{j+1}^{m}$. Thus each connected component of the graph is a tree rooted at time $t=1$ which grows in the negative direction in $t$. Hence each connected component of $Z$ is a 2-dimensional disk.

In the case where $2 m=6$, we see in a similar way that $Z \subset[0,1] \times \sigma_{j+1}^{3}$ is an immersed image of 2-dimensional disks which has generically a finite number of double points. That is, the curves joining the pairs of intersection points in $\left(h^{\prime}{ }_{t}^{(j)}\right)^{-1}\left(k\left(\tau^{3}\right)\right)$ may intersect at finitely many points $\left(\hat{t}_{\ell}, \hat{x}_{\ell}\right)\left(\ell=1, \ldots, \hat{r}^{(j)}\right)$. Then for generic $H^{\prime(j)}, \hat{t}_{\ell}$ are not the time of generations or cancellations. When two geodesic curves $\gamma_{1}^{(t)}$ and $\gamma_{2}^{(t)}$ intersect at the time $\hat{t}_{\ell}$, we modify one of the family $\left\{\gamma_{2}^{(t)}\right\}$ of geodesic curves near $\hat{t}_{\ell}$ by a family $\left\{\gamma_{2}^{\prime(t)}\right\}$ of curves which does not intersect $\left\{\gamma_{1}^{(t)}\right\}$ near $\hat{t}_{\ell}$.

More concretely, for a small positive real number $\hat{\varepsilon}_{\ell}$, we can find a neighborhood of $\gamma_{1}^{\left(\hat{t}_{\ell}\right)} \cup \gamma_{2}^{\left(\hat{t}_{\ell}\right)} \subset[0,1] \times \sigma^{m}$ which is diffeomorphic to $\left(\hat{t}_{\ell}-\hat{\varepsilon}_{\ell}, \hat{t}_{\ell}+\hat{\varepsilon}_{\ell}\right) \times X$, where $X$ is a neighborhood of $[-1,1] \times\{0\} \times\{0\} \cup\{0\} \times[-1,1] \times\{0\}$ in $\mathbb{R}^{3}$,

$$
\gamma_{1}^{\left(\hat{t}_{\ell}\right)}=\left\{\hat{t}_{\ell}\right\} \times[-1,1] \times\{0\} \times\{0\}
$$

and

$$
\gamma_{2}^{\left(\hat{t}_{\ell}\right)}=\left\{\hat{t}_{\ell}\right\} \times\{0\} \times[-1,1] \times\{0\}
$$

We can choose the parametrization in this neighborhood so that

$$
\gamma_{1}^{\left(\hat{t}_{\ell}+s\right)}(u)=\left(\hat{t}_{\ell}+s, u, 0, s\right)
$$

and

$$
\gamma_{2}^{\left(\hat{t}_{\ell}+s\right)}(u)=\left(\hat{t}_{\ell}+s, v_{1} s, u+v_{2} s, v_{3} s\right)
$$


for a vector $\left(v_{1}, v_{2}, v_{3}\right) \in \mathbb{R}^{3}\left(v_{3} \neq 1\right)$. By using a smooth bump function $\mu:[-1,1] \rightarrow[0,1]$ such that $\mu(x)=\mu(-x), \mu \mid[0,1 / 3]=1$ and $\mu \mid[2 / 3,1]=0$, we modify $\gamma_{2}^{(t)}$. Put

$$
\gamma_{2}^{\prime\left(\hat{t}_{\ell}+s\right)}(u)=\left(\hat{t}_{\ell}+s,\left(1+c_{\ell}\right) \mu\left(s / \hat{\varepsilon}_{\ell}\right) \mu\left(u / \delta_{\ell}\right)+v_{1} s, u+v_{2} s, v_{3} s\right),
$$

where $c_{\ell}$ and $\delta_{\ell}$ are small positive real numbers such that the image of $\left.\gamma_{2}^{\prime} \hat{t}_{\ell}+s\right)$ is contained in our neighborhood $X$. Then the curves $\gamma_{1}^{(t)}$ and $\gamma_{2}^{\prime(t)}\left(t \in\left(\hat{t}_{\ell}-\hat{\varepsilon}_{\ell}, \hat{t}_{\ell}+\hat{\varepsilon}_{\ell}\right)\right)$ do not intersect in $\sigma_{j+1}^{m}$.

Thus for $2 m \geq 6$, using the above family of curves if necessary, we have the union $Z^{\prime}$ of a finite number of disjointly embedded 2-dimensional disks in $[0,1] \times \sigma_{j+1}^{m}$ such that

$$
\left(H^{\prime(j)} \mid[0,1] \times \sigma_{j+1}^{m}\right)^{-1}\left(k\left(\tau^{m}\right)\right) \subset Z^{\prime} .
$$

Since $2 m \geq 6$, the images under generic $H^{\prime(j)}$ of these 2-dimensional disks are disjointly embedded in $M^{2 m} \backslash k\left(Q^{(m-1)}\right)$. The images of these disks are called the Whitney disks.

We have been looking at the intersection point set ${h^{\prime}}_{t}^{(j)}\left(\sigma_{j+1}^{m}\right) \cap k\left(\tau^{m}\right)$ for one $m$-dimensional cell $\tau^{m}$ of $Q^{m}$ other than $\sigma^{m *}$. These considerations can be applied to the intersection point sets ${h^{\prime}}_{t}^{(j)}\left(\sigma_{j+1}^{m}\right) \cap k\left(\tau^{m}\right)$ for all (finitely many) $m$-dimensional cells $\tau^{m}$ of $Q^{m}$ other than $\sigma^{m *}$ simultaneously. This is because, if $2 m \geq 8$, the embedded 2-dimensional disks $Z^{\prime}$ are disjoint for different $\tau^{m}$ for generic $H^{\prime(j)}$, and if $2 m=6$, we can remove the intersection of the embedded 2-dimensional disks $Z^{\prime}$ for different $\tau^{m}$ in a way similar to what we did for the intersection of $Z$ for the same $\tau^{m}$. Thus we obtained the union $Z^{\prime}$ of a finite number of disjointly embedded 2-dimensional disks in $[0,1] \times \sigma_{j+1}^{m}$ such that

$$
\left(H^{\prime(j)} \mid[0,1] \times \sigma_{j+1}^{m}\right)^{-1}\left(k\left(Q^{m} \backslash \sigma_{j+1}^{m *}\right)\right) \subset Z^{\prime},
$$

and $H^{\prime(j)} \mid Z^{\prime}$ is an embedding.

If $2 m \geq 8$, then the Whitney disks $H^{(j)}\left(Z^{\prime}\right)$ do not contain double points of $H^{\prime(j)}\left([0,1] \times P^{m}\right)$ for generic $H^{\prime(j)}$. This is because the inverse image of the double point set of $H^{\prime(j)}\left([0,1] \times P^{m}\right)$ is 2-dimensional in $[0,1] \times P^{m}$ and $m+1 \geq 5$.

If $2 m=6$, then the Whitney disks $H^{\prime(j)}\left(Z^{\prime}\right)$ may intersect the double point set of $H^{\prime(j)}\left([0,1] \times P^{3}\right)$. Then, for generic $H^{\prime(j)}$, the intersection is a finite set and we pick up the points of Whitney disks which are in the image of ${h^{\prime}}_{t}^{(j)}\left(P^{3}\right)$ with larger $t$;

$$
H^{\prime(j)}\left(t_{i}^{(j)}, w_{i}^{(j)}\right)=H^{\prime(j)}\left(t_{i}^{\prime(j)}, w_{i}^{\prime(j)}\right) \quad\left(i=1, \ldots, r^{(j)}\right),
$$

where $\left(t_{i}^{(j)}, w_{i}^{(j)}\right)$ is a point $Z^{\prime} \subset[0,1] \times \sigma_{j+1}^{m},\left(t^{\prime}{ }_{i}^{(j)}, w^{\prime}{ }_{i}^{(j)}\right) \in[0,1] \times P^{3}$ and $t_{i}^{(j)}<t^{\prime(j)}$. Then, for generic $H^{\prime(j)}$, the curve $H^{\prime(j)}\left(\left[t^{\prime}{ }_{i}^{(j)}, 1\right] \times\left\{{w^{\prime}}_{i}^{(j)}\right\}\right)$ is embedded in $M^{2 m} \backslash k\left(Q^{m}\right)$ and does not contain double points of $H^{\prime(j)}\left([0,1] \times P^{3}\right)$ other than 
$H^{\prime(j)}\left(t^{\prime(j)}, w_{i}^{\prime(j)}\right)$. Hence if $2 m=6$, we have the Whitney disks $H^{\prime(j)}\left(Z^{\prime}\right)$ together with the curves $H^{\prime(j)}\left(\left[t_{i}^{\prime(j)}, 1\right] \times\left\{w_{i}^{\prime(j)}\right\}\right)\left(i=1, \ldots, r^{\prime(j)}\right)$.

Using the Whitney disks $H^{\prime(j)}\left(Z^{\prime}\right)$ and curves $H^{\prime(j)}\left(\left[t^{\prime}{ }_{i}^{(j)}, 1\right] \times\left\{{w^{\prime}}_{i}^{(j)}\right\}\right)(i=1$, $\left.\ldots, r^{(j)}\right)$, we prove the following lemmas in the next section.

Lemma 3.6. For $h_{t}^{(j)}$, there is an isotopy $\left\{b_{t}^{(j+1)}\right\}_{t \in[0,1]}\left(b_{0}^{(j+1)}=\mathrm{id}\right)$ with support in a union of disjointly embedded open balls such that for $h_{t}^{\prime \prime(j)}=b_{t}^{(j+1)} \circ{h^{\prime}}_{t}^{(j)}$, $h_{t}^{\prime \prime(j)}$ is the identity on a neighborhood of $P^{(m-1)} \cup \bigcup_{i=1}^{j} \sigma_{i}^{m}$ and $h_{t}^{\prime \prime(j)}\left(\sigma_{j+1}^{m}\right) \cap$ $k\left(Q^{m} \backslash \sigma_{j+1}^{m *}\right)=\emptyset$.

Lemma 3.7. For $h_{t}^{\prime \prime(j)}$ given by Lemma 3.6, there are isotopies

$$
\left\{g_{t}^{(j+1)}\right\}_{t \in[0,1]} \subset \operatorname{Diff}_{c}^{r}\left(M^{2 m} \backslash k\left(Q^{m} \backslash \sigma_{j+1}^{m *}\right)\right) \quad\left(g_{0}^{(j+1)}=\mathrm{id}\right)
$$

and

$$
\left\{h_{t}^{(j+1)}\right\}_{t \in[0,1]} \subset \operatorname{Diff}_{c}^{r}\left(M^{2 m} \backslash\left(P^{(m-1)} \cup \bigcup_{i=1}^{j+1} \sigma_{i}^{m}\right)\right) \quad\left(h_{0}^{(j+1)}=\mathrm{id}\right)
$$

such that $h_{t}^{\prime \prime(j)}=g_{t}^{(j+1)} \circ h_{t}^{(j+1)}$.

Now we complete the proof of our main Theorem 1.2.

Proof of Theorem 1.2. Let $f$ be an element of $\operatorname{Diff}^{r}\left(M^{2 m}\right)_{0}$. By Lemma 3.2, there are $g \in \operatorname{Diff}_{c}^{r}\left(M^{2 m} \backslash k\left(Q^{m}\right)\right)_{0}$ and $h \in \operatorname{Diff}_{c}^{r}\left(M^{2 m} \backslash P^{(m-2)}\right)_{0}$ such that $f=g \circ h$. Then by using the approximation $\bar{h}$ of $h$,

$$
f=g \circ \bar{h} \circ\left(\bar{h}^{-1} \circ h\right) .
$$

By Lemmas 3.3 and 2.3, there are a diffeomorphism $a$ with support in a union of disjointly embedded open balls, $g^{\prime} \in \operatorname{Diff}_{c}^{r}\left(M^{2 m} \backslash k\left(Q^{m}\right)\right)_{0}$ and $h^{\prime \prime} \in \operatorname{Diff}_{c}^{r}\left(M^{2 m} \backslash\right.$ $\left.P^{(m-1)}\right)_{0}$ such that

$$
\bar{h}=a^{-1} \circ(a \circ \bar{h})=a^{-1} \circ g^{\prime} \circ h^{\prime \prime} .
$$

Put $h^{(0)}=h^{\prime \prime} \in \operatorname{Diff}_{c}^{r}\left(M^{2 m} \backslash P^{(m-1)}\right)_{0}$, and for $h^{(j)} \in \operatorname{Diff}_{c}^{r}\left(M^{2 m} \backslash\left(P^{(m-1)} \cup\right.\right.$ $\left.\left.\bigcup_{i=1}^{j} \sigma_{i}^{m}\right)\right)_{0}(j=0, \ldots, N-1)$, we use its approximation $\bar{h}^{(j)}$ and by Lemmas 3.4, 3.6 and 3.7, there are diffeomorphisms $a^{(j+1)}$ and $b^{(j+1)}$ with support in unions of disjointly embedded open balls, $g^{(j+1)} \in \operatorname{Diff}_{c}^{r}\left(M^{2 m} \backslash k\left(Q^{m} \backslash \sigma_{j+1}^{m *}\right)\right)_{0}$ and $h^{(j+1)} \in \operatorname{Diff}_{c}^{r}\left(M^{2 m} \backslash\left(P^{(m-1)} \cup \bigcup_{i=1}^{j+1} \sigma_{i}^{m}\right)\right)_{0}$ such that

$$
\begin{aligned}
h^{(j)} & =\bar{h}^{(j)} \circ\left(\left(\bar{h}^{(j)}\right)^{-1} \circ h^{(j)}\right) \\
& =\left(a^{(j+1)}\right)^{-1} \circ\left(a^{(j+1)} \circ \bar{h}^{(j)}\right) \circ\left(\left(\bar{h}^{(j)}\right)^{-1} \circ h^{(j)}\right) \\
& =\left(a^{(j+1)}\right)^{-1} \circ\left(b^{(j+1)}\right)^{-1} \circ g^{(j+1)} \circ h^{(j+1)} \circ\left(\left(\bar{h}^{(j)}\right)^{-1} \circ h^{(j)}\right) .
\end{aligned}
$$


Hence,

$$
\begin{aligned}
f= & g \circ \bar{h} \circ\left(\bar{h}^{-1} \circ h\right) \\
= & g \circ a^{-1} \circ g^{\prime} \circ h^{(0)} \circ\left(\bar{h}^{-1} \circ h\right) \\
= & g \circ a^{-1} \circ g^{\prime} \circ\left(a^{(1)}\right)^{-1} \circ\left(b^{(1)}\right)^{-1} \circ g^{(1)} \circ h^{(1)} \circ\left(\left(\bar{h}^{(0)}\right)^{-1} \circ h^{(0)}\right) \circ\left(\bar{h}^{-1} \circ h\right) \\
= & g \circ a^{-1} \circ g^{\prime} \circ\left(a^{(1)}\right)^{-1} \circ\left(b^{(1)}\right)^{-1} \circ g^{(1)} \circ \cdots \circ\left(a^{(N)}\right)^{-1} \circ\left(b^{(N)}\right)^{-1} \circ g^{(N)} \\
& \circ h^{(N)} \circ\left(\left(\bar{h}^{(N-1)}\right)^{-1} \circ h^{(N-1)}\right) \circ \cdots \circ\left(\left(\bar{h}^{(0)}\right)^{-1} \circ h^{(0)}\right) \circ\left(\bar{h}^{-1} \circ h\right) .
\end{aligned}
$$

Here, note that

$$
h^{(N)} \in \operatorname{Diff}_{c}^{r}\left(M^{2 m} \backslash\left(P^{(m-1)} \cup \bigcup_{i=1}^{N} \sigma_{i}^{m}\right)\right)_{0}=\operatorname{Diff}_{c}^{r}\left(M^{2 m} \backslash P^{m}\right)_{0}
$$

Since

$$
\left(\left(\bar{h}^{(N-1)}\right)^{-1} \circ h^{(N-1)}\right) \circ \cdots \circ\left(\left(\bar{h}^{(0)}\right)^{-1} \circ h^{(0)}\right) \circ\left(\bar{h}^{-1} \circ h\right) \in \operatorname{Diff}^{r}\left(M^{2 m}\right)
$$

is close to the identity, by Remark 2.10 , it can be written as $\hat{h} \circ \hat{a} \circ \hat{g}$, where $\hat{h} \in$ $\operatorname{Diff}_{c}^{r}\left(M^{2 m} \backslash P^{m}\right)_{0}, \hat{g} \in \operatorname{Diff}_{c}^{r}\left(M^{2 m} \backslash k\left(Q^{m}\right)\right)_{0}$ and $\hat{a}$ is with support in a union of disjointly embedded open balls which is a neighborhood of the union of $m$ handles. Thus

$$
\begin{aligned}
f=g & \circ a^{-1} \circ g^{\prime} \circ\left(a^{(1)}\right)^{-1} \circ\left(b^{(1)}\right)^{-1} \circ g^{(1)} \circ \cdots \\
& \cdots \circ\left(a^{(N)}\right)^{-1} \circ\left(b^{(N)}\right)^{-1} \circ g^{(N)} \circ h^{(N)} \circ \hat{h} \circ \hat{a} \circ \hat{g} .
\end{aligned}
$$

Now by the construction, each of $a^{-1},\left(a^{(1)}\right)^{-1}, \ldots,\left(a^{(N)}\right)^{-1},\left(b^{(1)}\right)^{-1}, \ldots,\left(b^{(N)}\right)^{-1}$ can be written as one commutator with support in a union of disjointly embedded open balls. The diffeomorphism $\hat{a}$ can be written as a product of two commutators by Theorem 1.1 (1). The diffeomorphism $h^{(N)} \circ \hat{h} \in \operatorname{Diff}_{c}^{r}\left(M^{2 m} \backslash P^{m}\right)_{0}$ can be written as a product of two commutators in $\operatorname{Diff}_{c}^{r}\left(M^{2 m} \backslash P^{m}\right)_{0}$ by Theorem 1.1 (1). Each of the diffeomorphisms $g, g^{\prime}$ and $\hat{g} \in \operatorname{Diff}_{c}^{r}\left(M^{2 m} \backslash k\left(Q^{m}\right)\right)_{0}$ can also be written as a product of two commutators in $\operatorname{Diff}_{c}^{r}\left(M^{2 m} \backslash k\left(Q^{m}\right)\right)_{0}$ by Theorem 1.1 (1). By the property of the triangulation, the diffeomorphism $g^{(j)} \in \operatorname{Diff}_{c}^{r}\left(M^{2 m} \backslash k\left(Q^{m} \backslash \sigma_{j+1}^{m *}\right)\right)_{0}$ is supported on an open set which can be deformed in a neighborhood of the embedded $(m-1)$-dimensional complex $\iota\left(\left(P^{(m-1)} \cup \sigma_{j}^{m}\right) / \sigma_{j}^{m}\right)$, and hence $g^{(j)}$ can be written as a product of two commutators in $\operatorname{Diff}_{c}^{r}\left(M^{2 m} \backslash k\left(Q^{m} \backslash \sigma_{j+1}^{m *}\right)\right)_{0}$ by Theorem 1.1 (1). Thus $f$ can be written as a product of $4 N+11$ commutators. 


\section{Proofs of the lemmas}

We now give the proofs of the lemmas we used in the previous section to show Theorem 1.2.

Proof of Lemma 3.2. This follows from Lemma 2.7.

Proof of Lemma 3.3. The construction of $a_{t}$ is essentially due to Burago, Ivanov and Polterovich ([4]) and we wrote it in the proof of Lemma 2.9 which is Lemma 6.5 in [30]. However, we write it again here, for, we use this argument later again.

For $\bar{H}\left(s_{i}, v_{i}\right)$, we take a small neighborhood $U_{i}$ of $\bar{H}\left(\left[s_{i}, 1\right] \times\left\{v_{i}\right\}\right)$ diffeomorphic to the $(2 m)$-dimensional ball. We can take these $U_{i}$ to be disjoint.

The intersection of $U_{i}$ and $\bar{H}\left([0,1] \times P^{(m-1)}\right)$ or $k\left(Q^{m}\right)$ is described as follows. We put a coordinate

$$
\left(x_{1}, x_{2}, \ldots, x_{m}, x_{m+1}, \ldots, x_{2 m}\right) \in(-2,2)^{2 m}
$$

on $U_{i}$ such that, for $\varepsilon_{i}>0$,

$$
\begin{gathered}
k\left(Q^{m}\right) \cap U_{i}=\{0\} \times\{0\}^{m-1} \times(-2,2)^{m}, \\
\bar{H}\left(\left(s_{i}-2 \varepsilon_{i}\left(1-s_{i}\right), 1\right] \times\left\{v_{i}\right\}\right) \cap U_{i}=(-2,1] \times\{0\}^{2 m-1}, \quad \text { and } \\
\bar{h}_{s_{i}+t\left(1-s_{i}\right)}\left(P^{(m-1)}\right) \cap U_{i}=\{t\} \times(-2,2)^{m-1} \times\{0\}^{m} \quad\left(t \in\left[-\varepsilon_{i}, 1\right]\right) .
\end{gathered}
$$

Take an isotopy $\left\{a_{t}\right\}_{t \in[0,1]}$ with support in $\bigsqcup_{i=1}^{r} U_{i}$ such that, on each $U_{i}, a_{0}=\mathrm{id}$ and, for $\left(x_{1}, x_{2}, \ldots, x_{2 m}\right) \in\left[-\varepsilon_{i}, 1\right] \times[-1,1]^{2 m-1} \subset(-2,2)^{2 m}$,

$$
a_{t}\left(x_{1}, x_{2}, \ldots, x_{2 m}\right)=\left(x_{1}-\left(1+\varepsilon_{i}\right) t, x_{2}, \ldots, x_{2 m}\right) .
$$

Now $\left(a_{1} \circ \bar{h}_{1}\right)\left(P^{(m-1)}\right) \cap k\left(Q^{m}\right)=\emptyset$. Moreover, by changing the time parameter of the above $a_{t}$, we obtain an isotopy $a_{t}\left(a_{0}=\mathrm{id}\right)$ with support in $\bigsqcup_{i=1}^{r} U_{i}$ such that for $h_{t}^{\prime}=a_{t} \circ \bar{h}_{t}$,

$$
h_{t}^{\prime}\left(P^{(m-1)}\right) \cap k\left(Q^{m}\right)=\emptyset \quad(t \in[0,1]) .
$$

In fact, if we put

$$
t=s_{i}+u_{i}\left(1-s_{i}\right) \in\left[s_{i}-\varepsilon_{i}\left(1-s_{i}\right), 1\right], \quad \text { i.e., } u_{i} \in\left[-\varepsilon_{i}, 1\right],
$$

and look at $a_{\left(u_{i}+\varepsilon_{i}\right) /\left(1+\varepsilon_{i}\right)} \circ \bar{h}_{s_{i}+u_{i}\left(1-s_{i}\right)}$, then on $U_{i}$,

$$
\begin{aligned}
& \left(a_{\left(u_{i}+\varepsilon_{i}\right) /\left(1+\varepsilon_{i}\right)} \circ \bar{h}_{s_{i}+u_{i}\left(1-s_{i}\right)}\right)\left(\left\{-\varepsilon_{i}\right\} \times[-1,1]^{m-1} \times\{0\}^{m}\right) \\
& \quad=a_{\left(u_{i}+\varepsilon_{i}\right) /\left(1+\varepsilon_{i}\right)}\left(\left\{u_{i}\right\} \times[-1,1]^{m-1} \times\{0\}^{m}\right) \\
& \quad=\left\{u_{i}-\left(u_{i}+\varepsilon_{i}\right)\right\} \times[-1,1]^{m-1} \times\{0\}^{m} \\
& \quad=\left\{-\varepsilon_{i}\right\} \times[-1,1]^{m-1} \times\{0\}^{m} .
\end{aligned}
$$


Hence by using the above $a_{t}$ with appropriate time change, we obtain the desired isotopy $a_{t}$.

Note that $a_{1} \in \operatorname{Diff}_{c}^{r}\left(\bigsqcup_{i=1}^{r} U_{i}\right)_{0}$ can be taken as one commutator with support in $\bigsqcup_{i=1}^{r} U_{i}([31])$.

Proof of Lemma 3.4. The proof is similar to that of Lemma 3.3.

For $\bar{H}^{(j)}\left(s_{i}^{(j)}, v_{i}^{(j)}\right)$, we take a small neighborhood $U_{i}^{(j)}$ of $\bar{H}\left(\left[s_{i}^{(j)}, 1\right] \times\left\{v_{i}^{(j)}\right\}\right)$ diffeomorphic to the $(2 m)$-dimensional ball. We can take these $U_{i}^{(j)}$ to be disjoint.

The intersection of $U_{i}^{(j)}$ and $\bar{H}^{(j)}\left([0,1] \times P^{m}\right)$ or $k\left(Q^{(m-1)}\right)$ is described as follows. We put a coordinate

$$
\left(x_{1}, x_{2}, \ldots, x_{m+1}, x_{m+2}, \ldots, x_{2 m}\right) \in(-2,2)^{2 m}
$$

on $U_{i}^{(j)}$ such that, for $\varepsilon_{i}^{(j)}>0$,

$$
\begin{aligned}
& k\left(Q^{(m-1)}\right) \cap U_{i}^{(j)}=\{0\} \times\{0\}^{m} \times(-2,2)^{m-1}, \\
& \bar{H}\left(\left(s_{i}^{(j)}-2 \varepsilon_{i}^{(j)}\left(1-s_{i}^{(j)}\right), 1\right] \times\left\{v_{i}^{(j)}\right\}\right) \cap U_{i}^{(j)}=(-2,1] \times\{0\}^{2 m-1}, \quad \text { and }
\end{aligned}
$$

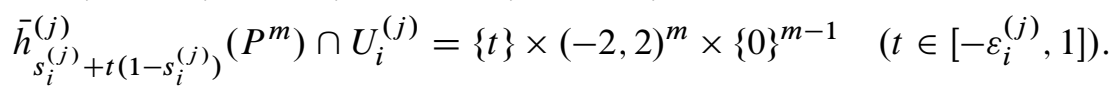

Take an isotopy $\left\{a_{t}^{(j+1)}\right\}_{t \in[0,1]}$ with support in $\bigsqcup_{i=1}^{r^{(j)}} U_{i}^{(j)}$ such that, on each $U_{i}^{(j)}$, $a_{0}^{(j+1)}=\mathrm{id}$ and, for $\left(x_{1}, x_{2}, \ldots, x_{2 m}\right) \in\left[-\varepsilon_{i}^{(j)}, 1\right] \times[-1,1]^{2 m-1} \subset(-2,2)^{2 m}$,

$$
a_{t}^{(j+1)}\left(x_{1}, x_{2}, \ldots, x_{2 m}\right)=\left(x_{1}-\left(1+\varepsilon_{i}^{(j)}\right) t, x_{2}, \ldots, x_{2 m}\right) \text {. }
$$

Now $\left(a_{1}^{(j+1)} \circ \bar{h}_{1}^{(j)}\right)\left(P^{m}\right) \cap k\left(Q^{(m-1)}\right)=\emptyset$. Moreover, by changing the time parameter, we obtain an isotopy $a_{t}^{(j+1)}\left(a_{0}^{(j+1)}=\mathrm{id}\right)$ with support in $\bigsqcup_{i=1}^{r} U_{i}^{(j)}$ such that, for ${h^{\prime}}_{t}^{(j)}=a_{t}^{(j+1)} \circ \bar{h}_{t}^{(j)}$,

$$
h_{t}^{\prime(j)}\left(P^{m}\right) \cap k\left(Q^{(m-1)}\right)=\emptyset \quad(t \in[0,1]) .
$$

In fact, if we put

$$
t=s_{i}^{(j)}+u_{i}^{(j)}\left(1-s_{i}^{(j)}\right) \in\left[s_{i}^{(j)}-\varepsilon_{i}^{(j)}\left(1-s_{i}^{(j)}\right), 1\right], \quad \text { i.e., } u_{i}^{(j)} \in\left[-\varepsilon_{i}^{(j)}, 1\right],
$$

and look at $a_{\left(u_{i}^{(j)}+\varepsilon_{i}^{(j)}\right) /\left(1+\varepsilon_{i}^{(j)}\right)}^{(j+1)} \circ \bar{h}_{s_{i}^{(j)}+u_{i}^{(j)}\left(1-s_{i}^{(j)}\right)}$, then on $U_{i}^{(j)}$,

$$
\begin{aligned}
& \left(a_{\left(u_{i}+\varepsilon_{i}\right) /\left(1+\varepsilon_{i}\right)}^{(j+1)} \circ \bar{h}_{s_{i}^{(j)}+u_{i}^{(j)}\left(1-s_{i}^{(j)}\right)}\right)\left(\left\{-\varepsilon_{i}^{(j)}\right\} \times[-1,1]^{m} \times\{0\}^{m-1}\right) \\
& \quad=a_{\left(u_{i}^{(j)}+\varepsilon_{i}^{(j)}\right) /\left(1+\varepsilon_{i}^{(j)}\right)}^{(j+1)}\left(\left\{u_{i}^{(j)}\right\} \times[-1,1]^{m} \times\{0\}^{m-1}\right) \\
& \quad=\left\{u_{i}^{(j)}-\left(u_{i}^{(j)}+\varepsilon_{i}^{(j)}\right)\right\} \times[-1,1]^{m} \times\{0\}^{m-1} \\
& =\left\{-\varepsilon_{i}^{(j)}\right\} \times[-1,1]^{m} \times\{0\}^{m-1} .
\end{aligned}
$$


Hence by using the above $a_{t}^{(j+1)}$ with appropriate time change, we obtain the desired isotopy $a_{t}^{(j+1)}$.

Note again that $a_{1}^{(j+1)} \in \operatorname{Diff}_{c}^{r}\left(\bigsqcup_{i=1}^{r^{(j)}} U_{i}^{(j)}\right)_{0}$ can be taken as one commutator with support in $\bigsqcup_{i=1}^{r^{(j)}} U_{i}^{(j)}([31])$.

Proof of Lemma 3.5. For

$$
F\left(t, x_{1}, \ldots, x_{m}\right)=\left(f_{1}\left(t, x_{1}, \ldots, x_{m}\right), \ldots, f_{m}\left(t, x_{1}, \ldots, x_{m}\right)\right),
$$

put

$$
\frac{\partial F}{\partial t}=\left(\begin{array}{c}
\frac{\partial f_{1}}{\partial t} \\
\vdots \\
\frac{\partial f_{m}}{\partial t}
\end{array}\right) \quad \text { and } \quad \frac{\partial F}{\partial x}=\left(\begin{array}{ccc}
\frac{\partial f_{1}}{\partial x_{1}} & \cdots & \frac{\partial f_{1}}{\partial x_{m}} \\
\vdots & \ddots & \vdots \\
\frac{\partial f_{m}}{\partial x_{1}} & \cdots & \frac{\partial f_{m}}{\partial x_{m}}
\end{array}\right) .
$$

On the 2-jet bundle $J^{2}\left(\mathbb{R} \times \mathbb{R}^{m}, \mathbb{R}^{m}\right)$, we consider the subbundle $E_{1}$ defined by $\operatorname{rank}\left(\frac{\partial F}{\partial t} \frac{\partial F}{\partial x}\right)=m-1$ and the subbundle $E_{2}$ defined by the two equations, $\operatorname{rank}\left(\frac{\partial F}{\partial x}\right)=m-1$ and rank $\left(\begin{array}{c}\frac{\partial F}{\partial x} \\ \frac{\partial}{\partial x} \operatorname{det} \frac{\partial F}{\partial x}\end{array}\right)=m-1$, where

$$
\frac{\partial}{\partial x} \operatorname{det} \frac{\partial F}{\partial x}=\left(\begin{array}{lll}
\frac{\partial}{\partial x_{1}} \operatorname{det} \frac{\partial F}{\partial x} & \cdots & \frac{\partial}{\partial x_{n}} \operatorname{det} \frac{\partial F}{\partial x}
\end{array}\right) .
$$

Then $E_{1}$ and $E_{2}$ are codimension 2 subbundles. The closures of these subbundles are the sets determined by the inequalities expressing the ranks are not greater than $m-1$.

By the jet transversality theorem, the jet of a generic map $F$ intersects these subbundles transversely. Hence the set

$$
\left\{(t, x) \mid J_{(t, x)}^{2} F \in E_{1} \cup E_{2}\right\}
$$

is an $(m-1)$-dimensional subset and its image in $\mathbb{R}^{m}$ is nowhere dense. We take a point $y$ in $\mathbb{R}^{m}$ in the complement of this image and consider its inverse image $F^{-1}(y)$. Then for a point $x \in F^{-1}(y)$, either rank $\left(\frac{\partial F}{\partial x}\right)=m$ holds or the three equations $\operatorname{rank}\left(\frac{\partial F}{\partial x}\right)=m-1, \operatorname{rank}\left(\frac{\partial F}{\partial t} \quad \frac{\partial F}{\partial x}\right)=m$ and rank $\left(\begin{array}{c}\frac{\partial F}{\partial x} \\ \frac{\partial}{\partial x} \operatorname{det} \frac{\partial F}{\partial x}\end{array}\right)=m$ hold.

If rank $\left(\frac{\partial F}{\partial x}\right)=m$ at $x$, then $x$ is a regular point of $F_{t}=F(t, \bullet)$ and the inverse image is locally a 1-dimensional manifold transverse to $\{t\} \times \mathbb{R}^{m}$.

Assume that the three equations hold. Since rank $\left(\frac{\partial F}{\partial x}\right)=m-1$, by the implicit function theorem, we can change the local coordinate $\left(x_{1}, \ldots, x_{m}\right)$ of the second factor of the source to $\left(x_{1}^{\prime}, \ldots, x_{m}^{\prime}\right)$ and that $\left(y_{1}, \ldots, y_{m}\right)$ of the target to $\left(y_{1}^{\prime}, \ldots, y_{m}^{\prime}\right)$ so that

$$
F\left(t, x_{1}^{\prime}, \ldots, x_{m}^{\prime}\right)=\left(x_{1}^{\prime}, \ldots, x_{m-1}^{\prime}, y_{m}^{\prime}\left(t, x_{1}^{\prime}, \ldots, x_{m}^{\prime}\right)\right) .
$$


Then $\operatorname{det}\left(\frac{\partial F}{\partial x}\right)=\frac{\partial y_{m}^{\prime}}{\partial x_{m}^{\prime}}$ and the matrix $\left(\begin{array}{c}\frac{\partial F}{\partial x} \\ \frac{\partial}{\partial x} \operatorname{det} \frac{\partial F}{\partial x}\end{array}\right)$ with respect to these coordinates is written as

$$
\left(\begin{array}{ccccc}
1 & 0 & \cdots & 0 & 0 \\
0 & \ddots & \ddots & \vdots & \vdots \\
\vdots & \ddots & \ddots & 0 & 0 \\
0 & \cdots & 0 & 1 & 0 \\
\frac{\partial y_{m}^{\prime}}{\partial x_{1}^{\prime}} & \cdots & \cdots & \frac{\partial y_{m}^{\prime}}{\partial x_{m}^{\prime}} & \frac{\partial y_{m}^{\prime}}{\partial x_{m}^{\prime}} \\
\frac{\partial^{2} y_{m}^{\prime}}{\partial x_{m}^{\prime} \partial x_{1}^{\prime}} & \cdots & \cdots & \frac{\partial^{2} y_{m}^{\prime}}{\partial x_{m}^{\prime} \partial x_{m-1}^{\prime}} & \frac{\partial^{2} y_{m}^{\prime}}{\partial x_{m}^{\prime}}
\end{array}\right)
$$

and the matrix $\left(\frac{\partial F}{\partial t} \quad \frac{\partial F}{\partial x}\right)$ with respect to these coordinates is written as

$$
\left(\begin{array}{cccccc}
0 & 1 & 0 & \cdots & 0 & 0 \\
0 & 0 & \ddots & \ddots & \vdots & \vdots \\
\vdots & \vdots & \ddots & \ddots & 0 & 0 \\
0 & 0 & \cdots & 0 & 1 & 0 \\
\frac{\partial y_{m}^{\prime}}{\partial t} & \frac{\partial y_{m}^{\prime}}{\partial x_{1}^{\prime}} & \cdots & \cdots & \frac{\partial y_{m}^{\prime}}{\partial x_{m-1}^{\prime}} & \frac{\partial y_{m}^{\prime}}{\partial x_{m}^{\prime}}
\end{array}\right) .
$$

Hence, $\frac{\partial y_{m}^{\prime}}{\partial x_{m}^{\prime}}=0, \frac{\partial y_{m}^{\prime}}{\partial t} \neq 0$ and $\frac{\partial^{2} y_{m}^{\prime}}{\partial x_{m}^{\prime}} \neq 0$ at $x$.

Thus at $x \in F^{-1}(y)$, either $\operatorname{det}\left(\frac{\partial F}{\partial x}\right) \neq 0$ or $F$ is locally written as

$$
F\left(t, x_{1}^{\prime}, \ldots, x_{m}^{\prime}\right)=\left(x_{1}^{\prime}, \ldots, x_{m-1}^{\prime}, y_{m}^{\prime}\left(t, x_{1}^{\prime}, \ldots, x_{m}^{\prime}\right)\right),
$$

where $\frac{\partial y_{m}^{\prime}}{\partial x_{m}^{\prime}}=0, \frac{\partial y_{m}^{\prime}}{\partial t} \neq 0$ and $\frac{\partial^{2} y_{m}^{\prime}}{\partial x_{m}^{\prime}} \neq 0$.

The proof of Lemma 3.6 is divided into two cases.

Proof of Lemma 3.6 in the case where $2 m \geq 8$. If $2 m \geq 8$, the Whitney disks guide the way to construct the isotopy $b_{t}^{(j+1)}$ with support in a union of disjoint open balls. In fact, the support of $b_{t}^{(j+1)}$ is in a neighborhood of the union of the Whitney disks. The construction of the isotopy $b_{t}^{(j+1)}$ is possible because the neighborhood of one of the Whitney disks can be considered as a neighborhood of a tree growing in the negative direction in $t$ in $[0,1] \times \sigma_{j+1}^{m}$.

The construction of $b_{t}^{(j+1)}$ is as follows. Take a vector field of the form $\frac{\partial}{\partial t}+\zeta(t, v)$ on the union of disks $Z^{\prime} \subset[0,1] \times \sigma_{j+1}^{m}$ which is tangent to $Z^{\prime}$ and transverse to the boundary $\partial Z^{\prime} \subset Z^{\prime}$, where $\zeta(t, v)$ is a vector field in the direction of $\sigma_{j+1}^{m}$. Such a vector field $\frac{\partial}{\partial t}+\zeta(t, v)$ exists because $Z^{\prime}$ deforms to a tree which grows in the negative direction in $t$ by shrinking the connected components of $Z^{\prime} \cap\left(\{t\} \times \sigma_{j+1}^{m}\right)$ to a point. We extend $\zeta(t, \bullet)$ on $\sigma_{j+1}^{m}$ so that the support is contained in a small neighborhood 
of $Z^{\prime}$. Let $b_{t}^{\prime(j+1)}$ denote the isotopy generated by $\frac{\partial}{\partial t}+\zeta(t, v)$. Then the support of $b^{\prime(j+1)}$ is contained in a neighborhood $U^{\prime(j)}$ of the union of the Whitney disks $H^{\prime(j)}\left(Z^{\prime}\right)$. Since $H^{\prime(j)}\left(Z^{\prime}\right)$ does not contain double points of $H^{\prime(j)}\left([0,1] \times P^{m}\right)$, the support of ${b^{\prime}}_{t}^{(j+1)}$ intersects $H^{\prime(j)}\left([0,1] \times P^{m}\right)$ only in $U^{\prime(j)}$. Here, $U^{\prime(j)}$ is a union of disjointly embedded open balls in $M^{2 m}$. Moreover, $\left(h^{\prime}{ }_{t}^{(j)}\right)_{*} \zeta(t, \bullet)$ is tangent to the union of the Whitney disks $H^{\prime(j+1)}\left(Z^{\prime}\right)$ in $M^{2 m}$ and

$$
\left(b_{t}^{\prime(j+1)}\right)^{-1}\left({h^{\prime}}_{t}^{(j)}\left(\sigma_{j+1}^{m}\right)\right) \cap k\left(Q^{m} \backslash \sigma_{j+1}^{m *}\right)=\emptyset \quad(t \in[0,1]) .
$$

Put $b_{t}^{(j+1)}=\left(b_{t}^{\prime(j+1)}\right)^{-1}$, then

$$
\left(b_{t}^{(j+1)} \circ{h^{\prime}}_{t}^{(j)}\right)\left(\sigma_{j+1}^{m}\right) \cap k\left(Q^{m} \backslash \sigma_{j+1}^{m *}\right)=\emptyset \quad(t \in[0,1]) .
$$

Note that $b_{1}^{(j+1)} \in \operatorname{Diff}_{c}^{r}\left(U^{\prime(j)}\right)_{0}$ can be taken as one commutator with support in $U^{\prime(j)}([31])$.

Proof of Lemma 3.6 in the case where $2 m=6$. If $2 m=6$, then we also consider the curves $H^{\prime(j)}\left(\left[t^{\prime(j)}, 1\right] \times\left\{w_{i}^{\prime(j)}\right\}\right)\left(i=1, \ldots, r^{\prime(j)}\right)$.

First take a small neighborhood $U^{\prime(j)}$ of the union of the Whitney disks which is a union of disjointly embedded open balls in $M^{6}$, and construct $b_{t}^{(j+1)}$ as in the case where $2 m \geq 8$. Then we modify it by using an isotopy.

We take a small neighborhood $U_{i}^{\prime(j)}$ of the curve $H^{\prime(j)}\left(\left[t_{i}^{\prime(j)}, 1\right] \times\left\{w_{i}^{\prime(j)}\right\}\right)(i=1$, $\left.\ldots, r^{\prime(j)}\right)$. We put a coordinate

$$
\left(x_{1}, x_{2}, x_{3}, x_{4}, x_{5}, x_{6}\right) \in(-2,3) \times(-2,2)^{5}
$$

on $U_{i}^{\prime(j)}$ such that, for $\varepsilon_{i}^{(j)}>0$,

$$
H^{\prime(j)}\left(\left(t_{i}^{\prime(j)}-2{\varepsilon^{\prime}}_{i}^{(j)}\left(1-{t^{\prime}}_{i}^{(j)}\right), 1\right] \times\left\{{w_{i}^{\prime}}_{i}^{(j)}\right\}\right) \cap{U^{\prime}}_{i}^{(j)}=(-2,1] \times\{0\}^{5},
$$

and

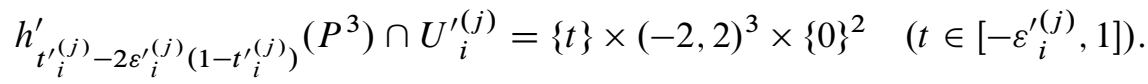

We take an isotopy $\left\{a_{t}^{\prime(j+1), i}\right\}_{t \in[0,1]}$ with support in ${U^{\prime}}_{i}^{(j)}$ such that $a_{0}^{\prime(j+1), i}=\mathrm{id}$ and, for $\left(x_{1}, x_{2}, x_{3}, x_{4}, x_{5}, x_{6}\right) \in\left[-\varepsilon^{\prime}{ }_{i}^{(j)}, 1\right] \times[-1,1]^{5} \subset(-2,3) \times(-2,2)^{5}$,

$$
a_{t}^{\prime(j+1), i}\left(x_{1}, x_{2}, x_{3}, x_{4}, x_{5}, x_{6}\right)=\left(x_{1}+t\left(1+\varepsilon_{i}^{\prime(j)}\right), x_{2}, x_{3}, x_{4}, x_{5}, x_{6}\right) .
$$

Put $\bar{a}=\prod_{i=1}^{r^{\prime(j)}} a_{1}^{\prime(j+1), i}$. Then $\bar{a} \circ b_{1}^{(j+1)} \circ \bar{a}^{-1}$ is isotopic to the identity by the isotopy with support in the union of disjoint 6-dimensional open balls $\bar{a}\left(U^{\prime(j)}\right)$. By the construction,

$$
\left(\left(\bar{a} \circ b_{1}^{(j+1)} \circ \bar{a}^{-1}\right) \circ \bar{h}_{1}\right)\left(\sigma_{j+1}^{3}\right) \cap k\left(Q^{3} \backslash \sigma_{j+1}^{3 *}\right)=\emptyset .
$$


Moreover, by an appropriate change of time parameter on each $U_{i}^{\prime(j)}$, we obtain an isotopy $\bar{a}_{t}(t \in[0,1])$ such that

$$
\left(\left(\bar{a}_{t} \circ b_{t}^{(j+1)} \circ \bar{a}_{t}^{-1}\right) \circ \bar{h}_{t}\right)\left(\sigma_{j+1}^{3}\right) \cap k\left(Q^{3} \backslash \sigma_{j+1}^{3 *}\right)=\emptyset
$$

and the support of the isotopy $\bar{a}_{t} \circ b_{t}^{(j+1)} \circ \bar{a}_{t}^{-1}$ is contained in $U^{\prime(j)} \cup \bigsqcup_{i=1}^{r^{\prime(j)}} U_{i}^{\prime(j)}$ which is a union of disjointly embedded open balls in $M^{2 m}$. Thus we obtained the desired isotopy.

Note that $\bar{a} \circ b_{1}^{(j+1)} \circ \bar{a}^{-1}$ can be taken as one commutator with support in a union of disjointly embedded open balls.

Proof of Lemma 3.7. This follows from Lemmas 3.6 and 2.3.

\section{Uniform simplicity}

We prove Corollary 1.3. In Theorem 2.2 of [31], we showed the following theorem.

Theorem 5.1 ([31]). Let $M^{n}$ be the interior of a compact $n$-dimensional manifold with handle decomposition with handles of indices not greater than $(n-1) / 2$. Let $c$ be the order of the set of indices appearing in the handle decomposition. Then any element of $\operatorname{Diff}_{c}^{r}\left(M^{n}\right)_{0}(1 \leq r \leq \infty, r \neq n+1)$ can be written as a product of two commutators. Moreover, if $M^{n}$ is connected, any element of $\operatorname{Diff}_{c}^{r}\left(M^{n}\right)_{0}$ can be written as a product of $4 c+1$ commutators with support in embedded open balls.

In Section 3, we showed that any element $f \in \operatorname{Diff}^{r}\left(M^{2 m}\right)_{0}$ can be written as

$$
\begin{aligned}
f=g & \circ a^{-1} \circ g^{\prime} \circ\left(a^{(1)}\right)^{-1} \circ\left(b^{(1)}\right)^{-1} \circ g^{(1)} \circ \cdots \\
& \cdots \circ\left(a^{(N)}\right)^{-1} \circ\left(b^{(N)}\right)^{-1} \circ g^{(N)} \circ h^{(N)} \circ \hat{h} \circ \hat{a} \circ \hat{g} .
\end{aligned}
$$

Since a compact subset of a union of disjointly embedded open balls is contained in a larger embedded open ball, each of diffeomorphisms $a^{-1},\left(a^{(1)}\right)^{-1}, \ldots,\left(a^{(N)}\right)^{-1}$, $\left(b^{(1)}\right)^{-1}, \ldots,\left(b^{(N)}\right)^{-1}$ can be written as one commutator with support in an embedded open ball and the diffeomorphism $\hat{a}$ can be written as a product of two commutators with support in an embedded open ball. Now by Theorem 5.1, each of the diffeomorphisms $h^{(N)} \circ \hat{h} \in \operatorname{Diff}_{c}^{r}\left(M^{2 m} \backslash P^{m}\right)_{0}, g, g^{\prime}$ and $\hat{g} \in \operatorname{Diff}_{c}^{r}\left(M^{2 m} \backslash k\left(Q^{m}\right)\right)_{0}$, $g^{(j)} \in \operatorname{Diff}_{c}^{r}\left(M^{2 m} \backslash k\left(Q^{m} \backslash \sigma_{j+1}^{m *}\right)\right)_{0}$ is written as a product of $4 m+1$ commutators with support in embedded open balls. Hence $f$ is written as a product of $4(N+4) m+3 N+7$ commutators with support in embedded open balls.

Now Corollary 1.3 follows from the following lemma ([31], Lemma 3.1).

Lemma 5.2 ([31]). Let $M^{n}$ be a connected $n$-dimensional manifold. Let $g$ be a nontrivial element of $\operatorname{Diff}_{c}^{r}\left(M^{n}\right)_{0}$. Assume that $f \in \operatorname{Diff}_{c}^{r}\left(M^{n}\right)_{0}$ is written as a 
product of commutators $\left[a_{i}, b_{i}\right](i=1, \ldots, k) ; f=\left[a_{1}, b_{1}\right] \cdots\left[a_{k}, b_{k}\right]$, where $a_{i}$ and $b_{i}$ are with support in an embedded open ball $U_{i} \subset \overline{U_{i}} \subset M^{n}$. Then $f$ can be written as a product of $4 k$ conjugates of $g$ or $g^{-1}$.

Proof of Corollary 1.3. Let $g$ be a nontrivial element of $\operatorname{Diff}^{r}\left(M^{2 m}\right)_{0}(1 \leq r \leq \infty$, $r \neq 2 m+1)$. Since any element $f$ of $\operatorname{Diff}^{r}\left(M^{2 m}\right)_{0}$ can be written as a product of $4(N+4) m+3 N+7$ commutators with support in embedded open balls, by Lemma 5.2, $f$ can be written as a product of $16(N+4) m+12 N+28$ conjugates of $g$ or $g^{-1}$.

Remark 5.3. We showed in [31] that, for a compact connected $n$-dimensional manifold $M^{n}$ with handle decomposition without handles of the middle index $n / 2$, for any elements $f$ and $g$ of $\operatorname{Diff}^{r}\left(M^{n}\right)_{0} \backslash\{\mathrm{id}\}, f$ can be written as a product of at most $16 n+28$ conjugates of $g$ or $g^{-1}$. For such manifolds, the bound for the number of conjugates depends only on the dimension $n$. In Corollary 1.3, however, the bound for the number of conjugates may depend on the topology of $M^{2 m}$.

\section{Appendix}

In this section, we show two propositions. The first proposition constructs the Morse function adapted to a smooth triangulation of a compact manifold. The second proposition constructs a cellular decomposition adapted to a Morse function.

Proposition 6.1. Let $P$ be a smooth triangulation of a compact n-dimensional manifold $M^{n}$. Let $\operatorname{bsd}(P)$ denote the barycentric subdivision of $P$ and $P^{*}$ be the cell decomposition dual to $P$ of $M^{n}$. Then there is a Morse function $f$ on $M^{n}$ and a Riemannian metric on $M^{n}$ such that, for the gradient flow $\varphi_{t}$ of $f$, there is a homeomorphism of $M^{n}$ which sends the stratification by the stable manifolds of the critical points of $f$ and that by the unstable manifolds of the critical points of $f$ to $P$ and $P^{*}$, respectively.

First we prepare a Morse type function on each simplex of bsd $(P)$.

Let $e_{i}(i=1, \ldots, n)$ be the basis of $\mathbb{R}^{n}$. Let

$$
\Delta^{n}=\left\{\left(t_{1}, \ldots, t_{n}\right)=\sum_{i=1}^{n} t_{i} e_{i} \in \mathbb{R}^{n} \mid 1 \geq t_{1} \geq \cdots \geq t_{n} \geq 0\right\}
$$

be the standard simplex. Let $f: \mathbb{R}^{n} \rightarrow \mathbb{R}$ be the function defined by

$$
f\left(t_{1}, \ldots, t_{n}\right)=n-\sum_{i=1}^{n} \cos \left(t_{n} / \pi\right) .
$$

The function $f$ is a Morse function such that the vertex $(1, \ldots, 1,0, \ldots, 0)=$ $\sum_{i=1}^{j} e_{i}$ of $\Delta^{n}$ is the critical point of index $j(j=0,1, \ldots, n)$ and $f\left(\sum_{i=1}^{j} e_{i}\right)=j$. 
Let $X_{\mathbb{R}^{n}}=\operatorname{grad}(f)$ denote the gradient vector field of $f$ with respect to the Euclidean metric. The standard simplex is invariant under the flow generated by $X_{\mathbb{R}^{n}}$.

Let $\operatorname{bsd}(P)$ be the barycentric subdivision of $P$. An $n$-dimensional simplex of $\operatorname{bsd}(P)$ is the simplex with vertices $b_{\sigma^{0}}, \ldots, b_{\sigma^{n}}$, where $\sigma^{0} \prec \sigma^{1} \prec \cdots \prec \sigma^{n-1} \prec \sigma^{n}$, $b_{\sigma^{j}}$ is the barycenter of the $j$-dimensional simplex $\sigma^{j}$ and " $\sigma^{i} \prec \sigma^{j}$ "means that " $\sigma^{i}$ is a face of $\sigma^{j}$ ".

Let $g: M^{n} \rightarrow \Delta^{n}$ be the map which sends each $n$-dimensional simplex with vertices $b_{\sigma^{0}}, \ldots, b_{\sigma^{n}}$ of $\operatorname{bsd}(P)$ linearly to $\Delta^{n}$ so that $g\left(b_{\sigma^{j}}\right)=\sum_{i=0}^{j} e_{i}$. Then $f \circ g$ is a piecewise smooth function on $M^{n}$ which looks like a Morse function on $M^{n}$ and $X=g_{*}^{-1} X_{\mathbb{R}^{n}}$ is a Lipschitz continuous piecewise smooth vector field on $M^{n}$.

We show that there are a Morse function $\hat{f}: M^{n} \rightarrow \mathbb{R}$ and a Riemannian metric on $M^{n}$ such that there is a homeomorphism of $M^{n}$ sending the stratification by the stable manifolds for the gradient flow of the critical points of $\hat{f}$ to the triangulation $P$ and the stratification by the unstable manifolds of the critical points of $\hat{f}$ to the dual cell decomposition $P^{*}$.

Since the function $f \circ g$ is transverse to the triangulation outside a neighborhood of the set of vertices of bsd $(P),(f \circ g)^{-1}(k+1 / 2)$ is a piecewise smooth codimension 1 submanifold of $M$ "transverse" to each simplex of bsd $(P)$ and is transverse to the vector field $X$.

We are going to modify $(f \circ g)^{-1}(k+1 / 2)$ to a smooth manifold $M_{k+1 / 2}$ transverse to each simplex of $\operatorname{bsd}(P)$ and to the vector field $X$.

Let bsd $(P)^{(i)}$ denote the $i$-dimensional skeleton of bsd $(P)$. First, we modify $(f \circ$ $g)^{-1}(k+1 / 2)$ in a neighborhood of the intersection $(f \circ g)^{-1}(k+1 / 2) \cap \operatorname{bsd}(P)^{(1)}$ and obtain $M_{k+1 / 2}^{(1)} \subset M^{n}$ approximating $(f \circ g)^{-1}(k+1 / 2)$ which is smooth near the 1-dimensional skeleton bsd $(P)^{(1)}$ and transverse to bsd $(P)^{(1)}$ and to the vector field $X$. After obtaining $M_{k+1 / 2}^{(i)} \subset M^{n}$ which is smooth near the $i$-dimensional skeleton $\operatorname{bsd}(P)^{(i)}$ and transverse to $\operatorname{bsd}(P)^{(i)}$ and to the vector field $X$, we obtain $M_{k+1 / 2}^{(i+1)}$ approximating $M_{k+1 / 2}^{(i)}$ in a neighborhood of the intersection $M_{k+1 / 2}^{(i)} \cap \operatorname{bsd}(P)^{(i+1)}$ which is smooth near the $(i+1)$-dimensional skeleton $\operatorname{bsd}(P)^{(i+1)}$ and transverse $\operatorname{bsd}(P)^{(i+1)}$ and to the vector field $X$. Finally, put $M_{k+1 / 2}=M_{k+1 / 2}^{(n-1)}$. Then $M_{k+1 / 2}$ is a smooth codimension 1 submanifold transverse to $\operatorname{bsd}(P)$ and $X$.

The codimension 1 submanifold $M_{k+1 / 2}$ divides $M^{n}$ into two compact manifolds $W_{k}$ and $W_{n-k}^{*}$ which are obtained from $(f \circ g)^{-1}([0, k+1 / 2])$ and $(f \circ g)^{-1}([k+$ $1 / 2, n]$ ) by smoothing, respectively.

We are going to show that $W_{k}$ is diffeomorphic to the manifold obtained from $W_{k-1}$ by attaching handles of index $k$ for $k$-dimensional simplices of $P$ and by smoothing along the corner. That is, for each $k$-dimensional simplex $\sigma^{k}$, we can define a handle $D_{\sigma^{k}}^{k} \times D_{\sigma^{k}}^{n-k}$ of index $k$ such that $W_{k}$ is diffeomorphic to the manifold obtained from $W_{k-1}$ by attaching $D_{\sigma^{k}}^{k} \times D_{\sigma^{k}}^{n-k}$ for all $k$-dimensional simplices $\sigma^{k}$ of $P$ and by 
smoothing along the corner. The reason is as follows. First, for each $k$-dimensional simplex $\sigma^{k}$ of $P$, since the intersection $S_{\sigma^{k}}^{k-1}=\sigma^{k} \cap M_{k-1 / 2}$ approximates $\sigma^{k} \cap$ $(f \circ g)^{-1}(k-1 / 2)$, it is diffeomorphic to the $(k-1)$-dimensional sphere $S^{k-1}$ which bounds a $k$-dimensional disk $D_{\sigma^{k}}^{k}$ in $\sigma^{k}$. Then by choosing a Riemannian metric in a neighborhood of $\sigma^{k}$ and using the exponential map, we obtain a diffeomorphism from a neighborhood of the zero section of the normal bundle of the $k$-dimensional disk $D_{\sigma^{k}}^{k}$ to a neighborhood of $D_{\sigma^{k}}^{k}$. By an appropriate choice of the metric, this defines an embedding $D_{\sigma^{k}}^{k} \times D_{\sigma^{k}}^{n-k} \subset M^{n}$ such that $\partial D_{\sigma^{k}}^{k} \times D_{\sigma^{k}}^{n-k} \subset M_{k-1 / 2}$. Then we obtain

$$
W_{k}^{\prime}=W_{k-1} \cup \bigcup_{\sigma^{k}}\left(D_{\sigma^{k}}^{k} \times D_{\sigma^{k}}^{n-k}\right) \quad\left(\subset W_{k}\right)
$$

We can add a neighborhood of the corner of $W_{k}^{\prime}$ and obtain $W_{k}^{\prime \prime}$ such that the orbits of $\psi_{t}$ on $W_{k}-\operatorname{int}\left(W_{k}^{\prime \prime}\right)$ are transverse to $M_{k+1 / 2}=\partial W_{k}$ and $\partial W_{k}^{\prime \prime}$. Here each orbit of $\psi_{t}$ intersects both $\partial W_{k}^{\prime \prime}$ and $\partial W_{k}$. Since this transversality is preserved when we approximate $X$ by a smooth vector field $\hat{X}, W_{k}-\operatorname{int}\left(W_{k}^{\prime \prime}\right)$ is diffeomorphic to $M_{k+1 / 2} \times[0,1]$. Thus this gives the $(n-k-1)$-dimensional sphere $S_{\sigma^{k}}^{n-k-1}$ on $M_{k+1 / 2}=\partial W_{k}$ corresponding to $\{0\} \times \partial D_{\sigma^{k}}^{n-k}$ which will be used as the belt sphere.

Now we define a smooth vector field $\widehat{X}$ on $M^{n}$ which generates the flow $\hat{\psi}_{t}$ satisfying the following conditions.

(1) $\hat{X}$ restricted to a neighborhood of $b_{\sigma^{k}}$ is of the form

$$
-\sum_{i=1}^{k} x_{i} \frac{\partial}{\partial x_{i}}+\sum_{i=k+1}^{n} x_{i} \frac{\partial}{\partial x_{i}}
$$

and the stable manifold $L_{b\left(\sigma^{k}\right)}^{s}$ of $b_{\sigma^{k}}$ of the flow $\hat{\psi}_{t}$ contains $D_{\sigma^{k}}^{k} \times\{0\} \subset \sigma^{k}$.

(2) The orbits of $\hat{\psi}_{t}$ are transverse to $M_{k+1 / 2}(k=0, \ldots, n-1)$.

(3) An orbit of $\hat{\psi}_{t}$ in $W_{k} \backslash \operatorname{int}\left(W_{k-1}\right)$ is one of the following types.

- An orbit crossing through both $M_{k-1 / 2}$ and $M_{k+1 / 2}$.

- An orbit contained in the stable manifold of $b_{\sigma^{k}}$ and crossing through $M_{k-1 / 2}$ at a point of $S_{\sigma^{k}}^{k-1}$.

- An orbit contained in the unstable manifold of $b_{\sigma^{k}}$ and crossing through $M_{k+1 / 2}$ at a point of $S_{\sigma^{k}}^{n-k-1}$.

(4) For two simplices $\sigma^{k}$ and $\sigma^{k+1}$ of $P$, if $\sigma^{k} \prec \sigma^{k+1}$, then $S_{\sigma^{k}}^{n-k-1}$ and $S_{\sigma^{k+1}}^{k}$ $\left(\subset M_{k+1 / 2}\right)$ intersect transversely at a point. Conversely, if $S_{\sigma^{k}}^{n-k-1}$ and $S_{\sigma^{k+1}}^{k}$ $\left(\subset M_{k+1 / 2}\right)$ intersect, they intersect transversely, and $\sigma^{k} \prec \sigma^{k+1}$. 
The statement (4) implies that there is a unique orbit of $\hat{\psi}_{t}$ connecting $b_{\sigma^{k}}$ and $b_{\sigma^{k+1}}$ if and only if $\sigma^{k} \prec \sigma^{k+1}$.

By [21], for this $\hat{\psi}_{t}$, we can define a Morse function $\hat{f}$ and a Riemannian metric such that $\operatorname{grad}(\hat{f})=\hat{X}$. These are the desired Morse function and Riemannian metric for our Proposition 6.1.

Proof of Proposition 6.1. We show that the configuration of stable manifolds and unstable manifolds of $\hat{\psi}_{t}$ is homeomorphic to the configuration of the triangulation $P$ and its dual cell decomposition $P^{*}$.

We explain how we take $W_{k}^{\prime}$ and $W_{k}^{\prime \prime}$ such that $W_{k-1} \subset W_{k}^{\prime} \subset W_{k}^{\prime \prime} \subset W_{k}$ related to the flow $\hat{\psi}_{t}$.

First, each connected component of $W_{0}$ is in the unstable manifold of a 0 dimensional simplex $\sigma^{0}$. On $M_{1 / 2}=\partial W_{0}$ we have a number of $S_{\sigma^{1}}^{0}$ for 1-dimensional simplices $\sigma^{1}$. Hence the stable manifold $L_{b\left(\sigma^{1}\right)}^{s}$ of $b_{\sigma^{1}}$ consists of $b_{\sigma^{1}}$ and the two orbits of $\hat{\psi}_{t}$ connecting $b_{\sigma^{1}}$ and $\sigma_{i}^{0}(i=1,2)$ such that $\sigma_{i}^{0} \prec \sigma^{1}$.

For a 1-dimensional simplex $\sigma^{1}$, in a neighborhood of $b_{\sigma^{1}}$, the unstable manifold $L_{b\left(\sigma^{1}\right)}^{u}$ of $b_{\sigma^{1}}$ divides the neighborhood into two parts which are the subsets of the unstable manifolds of $\sigma_{1}^{0} \prec \sigma^{1}$ and $\sigma_{2}^{0} \prec \sigma^{1}$. We can take the union of $W_{0}$ and appropriate closed neighborhoods of $L_{b\left(\sigma^{1}\right)}^{s}$ for 1-dimensional simplices $\sigma^{1}$ of $P$ as $W_{1}^{\prime}=W_{0} \cup \bigcup_{\sigma^{1}} D_{\sigma^{1}}^{1} \times D_{\sigma^{1}}^{n-1}$, where the flow $\hat{\psi}_{t}$ on $D_{\sigma^{1}}^{1} \times D_{\sigma^{1}}^{n-1}$ is of the form $\hat{\psi}_{t}\left(x_{1}, \ldots, x_{n}\right)=\left(e^{-t} x_{1}, e^{t} x_{2}, \ldots, e^{t} x_{n}\right)$. Then we can take $W_{1}^{\prime \prime}$ which is obtained from $W_{1}^{\prime}$ by smoothing along the corner and there is an isotopy sending $W_{1}^{\prime \prime}$ to $W_{1}$ along the orbits of $\hat{\psi}_{t}$. There is a homeomorphism $h_{1}$ sending $W_{1}$ to $(f \circ g)^{-1}([0,1+1 / 2])$ such that $h_{1}$ sends the stable manifold $L_{b\left(\sigma^{1}\right)}^{s}$ of $b_{\sigma^{1}}$ to $\sigma^{1}$ and the unstable manifold $L_{b\left(\sigma^{1}\right)}^{u}$ of $b_{\sigma^{1}}$ to $\sigma^{1 *} \cap(f \circ g)^{-1}([0,1+1 / 2])$, respectively.

Now we look at a 2-dimensional simplex $\sigma^{2}$. On $M_{1+1 / 2}$, we have $S_{\sigma^{2}}^{1}$ for each 2-dimensional simplex $\sigma^{2}$ of $P$ and $S_{\sigma^{1}}^{n-2}$ for each 1-dimensional simplex $\sigma^{1}$ of $P$. A 2-dimensional simplex $\sigma^{2}$ of $P$ has three faces $\sigma_{i}^{1}(i=1,2,3)$, hence we have three orbits of $\hat{\psi}_{t}$ which pass through $S_{\sigma^{2}}^{1} \cap S_{\sigma_{i}^{1}}^{n-2}$ and connect $b_{\sigma_{i}^{1}}$ and $b_{\sigma^{2}}(i=1,2$, 3). Each component of $S_{\sigma^{2}}^{1} \backslash \bigcup_{i=1}^{3} S_{\sigma^{2}}^{1} \cap S_{\sigma_{i}^{1}}^{n-2}$ is sent by the flow $\hat{\psi}_{t}$ in the negative time direction to one of the components of $W_{0}$. The component of $W_{0}$ is necessarily the one which contains one of the three vertices of $\sigma^{2}$ and the stable manifold $L_{b\left(\sigma^{2}\right)}^{s}$ of $b_{\sigma^{2}}$ is bounded by the union of stable manifolds of $b_{\sigma_{i}^{1}}(i=1,2,3)$ and the vertices of $\sigma^{2}$. Thus the stable manifold $L_{b\left(\sigma^{2}\right)}^{s}$ is homeomorphic to a 2-dimensional simplex and the union $\bigcup_{i \leq 2} L_{b\left(\sigma^{i}\right)}^{s}$ is homeomorphic to the 2-dimensional skeleton $P^{(2)}$. Then the stable manifold $L_{b\left(\sigma^{2}\right)}^{s}$ as well as a neighborhood of $L_{b\left(\sigma^{2}\right)}^{s}$ is divided by the union of the unstable manifolds of $b_{\sigma_{i}^{1}}(i=1,2,3)$ and $b_{\sigma^{2}}$ into three parts, 
each of which is contained in the stable manifold of one of the vertices of $\sigma^{2}$. We can take the union of $W_{1}$ and closed neighborhoods of $L_{b\left(\sigma^{2}\right)}^{s}$ for 2-dimensional simplices $\sigma^{2}$ of $P$ as $W_{2}^{\prime}=W_{1} \cup \bigcup_{\sigma^{2}}\left(D_{\sigma^{2}}^{2} \times D_{\sigma^{2}}^{n-2}\right)$, where the flow $\hat{\psi}_{t}$ on $D_{\sigma^{2}}^{2} \times D_{\sigma^{2}}^{n-2}$ is of the form $\hat{\psi}_{t}\left(x_{1}, \ldots, x_{n}\right)=\left(e^{-t} x_{1}, e^{-t} x_{2}, e^{t} x_{3}, \ldots, e^{t} x_{n}\right)$. We can take $W_{2}^{\prime \prime}$ which is obtained from $W_{2}^{\prime}$ by smoothing along the corner and there is an isotopy sending $W_{2}^{\prime \prime}$ to $W_{2}$ along the orbits of $\hat{\psi}_{t}$. Then there is a homeomorphism $h_{2}$ sending $W_{2}$ to $(f \circ g)^{-1}([0,2+1 / 2])$ extending $h_{1}$ such that $h_{2}$ sends the stable manifold $L_{b\left(\sigma^{2}\right)}^{s}$ of $b_{\sigma^{2}}$ to $\sigma^{2}$ and the unstable manifold $L_{b\left(\sigma^{2}\right)}^{u}$ of $b_{\sigma^{2}}$ to $\sigma^{2 *} \cap(f \circ g)^{-1}([0,2+1 / 2])$, respectively.

Inductively, assume that we showed that

(1) for a $(j-1)$-dimensional simplex $\sigma^{j-1}$ of $P$, the stable manifold of $b_{\sigma^{j-1}}$ is bounded by the union of the stable manifolds $L_{b\left(\sigma^{i}\right)}^{s}$ of $b_{\sigma^{i}}$ such that $\sigma^{i} \prec \sigma^{j-1}$,

(2) $L_{b\left(\sigma^{j-1}\right)}^{s}$ is homeomorphic to a $(j-1)$-dimensional simplex,

(3) the union $\bigcup_{i \leq j-1} L_{b\left(\sigma^{i}\right)}^{s}$ is homeomorphic to the $(j-1)$-dimensional skeleton $P^{(j-1)}$

(4) $L_{b\left(\sigma^{j-1}\right)}^{s}$ as well as a neighborhood of $L_{b\left(\sigma^{j-1}\right)}^{s}$ is divided by the union of the unstable manifolds $L_{b\left(\sigma^{i}\right)}^{u}$ of $b_{\sigma^{i}}$ such that $\sigma^{i} \prec \sigma^{j-1}$ into $j$ parts each of which is contained in the unstable manifold of one of the vertices of $\sigma^{j-1}$, and

(5) there is a homeomorphism $h_{j-1}$ sending $W_{j-1}$ to $(f \circ g)^{-1}([0, j-1 / 2])$ such that $h_{j-1}$ sends the stable manifold $L_{b\left(\sigma^{i}\right)}^{s}$ of $b_{\sigma^{i}}$ to $\sigma^{i}$ and the unstable manifold $L_{b\left(\sigma^{i}\right)}^{u}$ of $b_{\sigma^{i}}$ to $\sigma^{i *} \cap(f \circ g)^{-1}([0, j-1 / 2])$, respectively.

Consider a $j$-dimensional simplex $\sigma^{j}$. On $M_{j-1 / 2}$, we have $S_{\sigma^{j}}^{j-1}$ for each $j$ dimensional simplex $\sigma^{j}$ of $P$ and $S_{\sigma^{j-1}}^{n-j}$ for each $(j-1)$-simplex $\sigma^{j-1}$ of $P$. A $j$-dimensional simplex $\sigma^{j}$ of $P$ has $j+1(j-1)$-dimensional faces $\sigma_{i}^{j-1}(i=1$, $\ldots, j+1)$, hence we have $j+1$ orbits of $\hat{\psi}_{t}$ which pass through $S_{\sigma^{j}}^{j-1} \cap S_{\sigma_{i}^{j-1}}^{n-j}$ and connect $b_{\sigma_{i}^{j-1}}$ and $b_{\sigma^{j}}(i=1, \ldots, j+1)$. Any point on $S_{\sigma^{j}}^{j-1}$ is in an unstable manifold $L_{b\left(\sigma^{k}\right)}^{u}$ of $b_{\sigma^{k}}$ for a $k$-dimensional simplex, where $k \leq j-1$. If $k=j-1$, it is one of the points $S_{\sigma^{j}}^{j-1} \cap S_{\sigma_{i}^{j-1}}^{n-j}$. The flow $\hat{\psi}_{t}$ transverse to $M_{j-1 / 2}$ sends a neighborhood of $W_{j-1} \cup P^{(j)}$ to a neighborhood of $W_{j}$. Hence a neighborhood of $S_{\sigma^{j}}^{j-1} \cap S_{\sigma_{i}^{j-1}}^{n-j} \in M_{j-1 / 2}$ is divided by the union of the unstable manifolds $L_{b\left(\sigma^{i}\right)}^{u}$ of $b_{\sigma^{i}}$ such that $\sigma^{i} \prec \sigma^{j-1}$ into $j$ parts, each of which is contained in the un stable manifold of one of the vertices of $\sigma^{j-1}$. This means that the closure of the stable manifold of $b_{\sigma^{j}}$ contains the union of the stable manifolds $L_{b\left(\sigma^{i}\right)}^{s}$ of $b_{\sigma^{i}}$ such that $\sigma^{i} \prec \sigma^{j}$. Since $\bigcup_{\sigma^{i} \prec \sigma^{j}} L_{b\left(\sigma^{i}\right)}^{s}$ is homeomorphic to $\partial \Delta^{j}$, by looking at the flow $\hat{\psi}_{t}$, 
we see that the stable manifold of $b_{\sigma^{j}}$ is bounded by the union of the stable manifolds $L_{b\left(\sigma^{i}\right)}^{s}$ of $b_{\sigma^{i}}$ such that $\sigma^{i} \prec \sigma^{j}$. We see then that $L_{b\left(\sigma^{j}\right)}^{s}$ is homeomorphic to a $j$ dimensional simplex and $L_{b\left(\sigma^{j}\right)}^{s}$ as well as a neighborhood of $L_{b\left(\sigma^{j}\right)}^{s}$ is divided by the union of the unstable manifolds of $b_{\sigma^{i}}$ such that $\sigma^{i} \prec \sigma^{j}$ into $j+1$ parts each of which is contained in the unstable manifold of one of the vertices of $\sigma^{j}$. We can take the union of $W_{j-1}$ and closed neighborhoods of $L_{b\left(\sigma^{j}\right)}^{s}$ for $j$-dimensional simplices $\sigma^{j}$ of $P$ as $W_{j}^{\prime}=W_{j-1} \cup \bigcup_{\sigma^{j}}\left(D_{\sigma^{j}}^{j} \times D_{\sigma^{j}}^{n-j}\right)$, where the flow $\hat{\psi}_{t}$ on $D_{\sigma^{j}}^{j} \times D_{\sigma^{j}}^{n-j}$ is of the form

$$
\hat{\psi}_{t}\left(x_{1}, \ldots, x_{n}\right)=\left(e^{-t} x_{1}, \ldots, e^{-t} x_{j}, e^{t} x_{j+1}, \ldots, e^{t} x_{n}\right) .
$$

We can take $W_{j}^{\prime \prime}$ which is obtained from $W_{j}^{\prime}$ by smoothing along the corner and there is an isotopy sending $W_{j}^{\prime \prime}$ to $W_{j}$ along the orbits of $\hat{\psi}_{t}$. Then there is a homeomorphism $h_{j}$ sending $W_{j}$ to $(f \circ g)^{-1}([0, j+1 / 2])$ extending $h_{j-1}$ such that $h_{j}$ sends the stable manifold $L_{b\left(\sigma^{i}\right)}^{s}$ of $b_{\sigma^{i}}$ to $\sigma^{i}$ and the unstable manifold $L_{b\left(\sigma^{i}\right)}^{u}$ of $b_{\sigma^{i}}$ to $\sigma^{i *} \cap(f \circ$ $g)^{-1}([0, j+1 / 2])$, respectively.

Thus we see that the configuration of stable manifolds and unstable manifolds of $\hat{\psi}_{t}$ is homeomorphic to the configuration of the triangulation $P$ and its dual cell decomposition $P^{*}$.

Now we construct a cellular decomposition adapted to a Morse function.

Let $M^{n}$ be a compact $n$-dimensional manifold. Let $F: M^{n} \rightarrow[0, n]$ be a selfindexing Morse function. Then there is a Riemannian metric such that the gradient flow $\varphi_{t}$ at a critical point of $F$ of index $k$ is of the form

$$
\varphi_{t}\left(x_{1}, \ldots, x_{n}\right)=\left(e^{-t} x_{1}, \ldots, e^{-t} x_{k}, e^{t} x_{k+1}, \ldots, e^{t} x_{n}\right)
$$

in a coordinate neighborhood and the stable manifolds and unstable manifolds of critical points of $F$ are transverse.

For such a gradient flow we have the following proposition.

Proposition 6.2. For a $k$-dimensional stable manifold $L$ of a critical point (of index $k$ ) of $F$, there is a continuous map $h: D^{k} \rightarrow M^{n}$ such that $h \mid \operatorname{Int}\left(D^{k}\right)$ is a diffeomorphism to $L$ and $h\left(\partial D^{k}\right) \subset P^{(k-1)}$, where $P^{(k-1)}$ is the $(k-1)$-dimensional skeleton of the stratification by the stable manifolds of $\varphi_{t}$.

This proposition is shown by Laudenbach in [13]. The author is grateful to the referee for indicating him this reference. We include the proof of Proposition 6.2 for completeness.

To show Proposition 6.2, we need to use the fact that the stratification by the stable manifolds of such $\varphi_{t}$ satisfy a much stronger condition, namely, the closure of a stable manifold is a submanifold with conical singularities (smcs) which is defined in [13]. 
An $m$-dimensional stratified subset $X=X^{(m)} \supset \cdots \supset X^{(0)}$ of $M^{n}$ defined in Section 2 is called a submanifold with conical singularities (smcs) if, for $1 \leq k \leq m$ and any $x \in X^{(k)} \backslash X^{(k-1)}$, there are a neighborhood $V$ of $x$ diffeomorphic to $D^{k} \times D^{n-k}$ and an $(m-k)$-dimensional smcs $T=T^{(m-k)} \supset \cdots \supset T^{(0)}$ in $D^{n-k}$ such that $V \cap X$ is diffeomorphic to $D^{k} \times T$, and for $x \in X^{(0)}$, there is a $C^{1}$ embedded $n$-dimensional ball $B$ centered at $x$ such that $X^{\prime}=X \cap \partial B$ is an $(m-1)$ dimensional smcs in the $(n-1)$-dimensional sphere and $\left(B, B \cap X^{(m)}, \cdots, B \cap\right.$ $\left.X^{(1)}\right)$ is diffeomorphic to $\left(B, C X^{\prime(m-1)}, \cdots, C X^{\prime(0)}\right)$, where $C$ denotes the cone with respect to the linear structure of the $C^{1}$ parametrization for $B$.

Roughly speaking Proposition 6.2 is shown in the following way. Let $p_{1}^{j}, \ldots, p_{c_{j}}^{j}$ be the critical points of $F$ of index $j$. Let $S_{p_{i}^{j}}^{j-1}$ denote the attaching sphere which is the intersection of the stable manifold $L_{p_{i}^{j}}^{s_{i}}$ and $M_{j-1 / 2}$, and is the boundary of the core disk $D_{p_{i}^{j}}^{j}=L_{p_{i}^{j}}^{s} \cap F^{-1}([j-1 / 2, j+1 / 2])$. Let $S_{p_{i}^{j}}^{n-j-1}$ denote the belt sphere which is the intersection of the unstable manifold $L_{p_{i}^{j}}^{u}$ and $M_{j+1 / 2}$, and is the boundary of the cocore disk $D_{p_{i}^{j}}^{n-j}=L_{p_{i}^{j}}^{u} \cap F^{-1}([j-1 / 2, j+1 / 2])$.

We look at $\bar{L} \cap M_{j+1 / 2}$ for $j=k-1, \ldots, 0$. and we show that $\bar{L} \cap M_{j+1 / 2}$ is a $(k-1)$-dimensional smcs of $M_{j+1 / 2}$. In fact, on $M_{j+1 / 2}$, there are belt spheres $S_{p_{i}^{j}}^{n-j-1}\left(i=1, \ldots c_{j}\right)$ which intersect transversely to $\bar{L} \cap M_{j+1 / 2}$. On the cocore disk $D_{p_{i}^{j}}^{n-j}$ which is bounded by $S_{p_{i}^{j}}^{n-j-1}, \bar{L} \cap D_{p_{i}^{j}}^{n-j}$ is homeomorphic to the cone over $\bar{L} \cap S_{p_{i}^{j}}^{n-j-1} . \bar{L}$ restricted to a neighborhood of the cocore disk $D_{p_{i}^{j}}^{n-j}$ is homeomorphic to a product of $\bar{L} \cap D_{p_{i}^{j}}^{n-j}$ and an open ball of $D^{j}$. Using the flow $\varphi_{t}$ on $F^{-1}([j-$ $1 / 2, j+1 / 2]) \backslash \bigcup_{i=1}^{c_{j}} D_{p_{i}^{j}}^{n-j}$, we see that $\bar{L} \cap M_{j-1 / 2}$ is a $(k-1)$-dimensional smcs of $M_{j-1 / 2}$.

By using this structure we define the homeomorphism $h$ in the proposition.

Now the first step of the proof of Proposition 6.2 is the following lemmas, which show that the closure of a stable manifold of such $\varphi_{t}$ is a submanifold with conical singularities (smcs) ([13], Proposition 2).

Lemma 6.3. Let $\varphi_{t}$ be the flow on $D^{j} \times D^{n-j}$ such that $\varphi_{t}(\boldsymbol{x}, \boldsymbol{y})=\left(e^{-t} \boldsymbol{x}, e^{t} \boldsymbol{y}\right)$, where $\boldsymbol{x}=\left(x_{1}, \ldots, x_{j}\right)$ and $\boldsymbol{y}=\left(x_{j+1}, \ldots, x_{n}\right)$. Let $N=N^{(k)} \supset \cdots \supset N^{(0)}$ be $a$ $k$-dimensional stratified subset of $D^{j} \times D^{n-j}$ invariant under the flow $\varphi_{t}$ such that $N \cap\left(D^{j} \times \partial D^{n-j}\right)$ is a $(k-1)$-dimensional smcs of $D^{j} \times \partial D^{n-j}$ near $\{0\} \times \partial D^{n-j}$ and $N$ is transverse to $\{0\} \times D^{n-j}$. Then there is a neighborhood $U$ of $0 \in D^{j}$ such that $N \cap\left(U \times D^{n-j}\right)$ is homeomorphic to $U \times C\left(N \cap\left(\{0\} \times \partial D^{n-j}\right)\right)$, where $C$ denotes the cone. 
Proof. Since $N \cap\left(D^{j} \times \partial D^{n-j}\right)$ is a $(k-1)$-dimensional smcs and transverse to $\{0\} \times \partial D^{n-j}, N^{\prime}=N \cap\left(\{0\} \times \partial D^{n-j}\right)$ is a $(k-1-j)$-dimensional smcs in $\{0\} \times \partial D^{n-j}$ and there is a positive real number $\varepsilon$ such that the $\varepsilon$-neighborhood $U=\operatorname{int}\left(D_{\varepsilon}^{j}\right)$ of $0 \in D^{j}$ has the following property. There is a mapping $v: U \times N^{\prime} \rightarrow$ $\partial D^{n-j}$ such that $\boldsymbol{v}(0, \boldsymbol{y})=\boldsymbol{y}$,

$$
N \cap\left(U \times \partial D^{n-j}\right)=\left\{(\boldsymbol{x}, \boldsymbol{v}(\boldsymbol{x}, \boldsymbol{y})) \mid(\boldsymbol{x}, \boldsymbol{y}) \in U \times N^{\prime}\right\},
$$

and $v$ is smooth on each product $U \times S$, where $S$ is a stratum of $N^{\prime}$. By the invariance under the flow $\varphi_{t}$, the set $\{(\boldsymbol{x}, \boldsymbol{v}(\boldsymbol{x}, \boldsymbol{y})) \mid(\boldsymbol{x}, \boldsymbol{y}) \in U \times S\}$ is contained in the stratum in $N \cap\left(U \times D^{n-j}\right)$ which is written as

$$
\{(\boldsymbol{x}, s \boldsymbol{v}(s \boldsymbol{x}, \boldsymbol{y})) \mid(\boldsymbol{x}, \boldsymbol{y}) \in U \times S, s \in[0,1]\} .
$$

In particular, $N \cap\left(\{0\} \times D^{n-j}\right)=C\left(N^{\prime}\right)$. Hence the map $(\boldsymbol{x}, s \boldsymbol{v}(s \boldsymbol{x}, \boldsymbol{y})) \longmapsto$ $(\boldsymbol{x}, \boldsymbol{s} \boldsymbol{v}(0, \boldsymbol{y}))$ is a homeomorphism sending $N \cap\left(U \times D^{n-j}\right)$ to $U \times C\left(N^{\prime}\right)$.

Lemma 6.4. $\bar{L} \cap M_{j+1 / 2}$ is a $(k-1)$-dimensional smes of $M_{j+1 / 2}$ for $j=k-1$, ..., 0 .

Proof. The above lemma implies that if $\bar{L} \cap M_{j+1 / 2}$ is a $(k-1)$-dimensional smcs of $M_{j+1 / 2}$, then on

$$
M_{j+1 / 2}^{\prime}=M_{j+1 / 2} \backslash \bigcup_{i=1}^{c_{j}} U \times \partial D_{p_{i}^{j}}^{n-j} \cup \bigcup_{i=1}^{c_{j}} \partial U \times D_{p_{i}^{j}}^{n-j}
$$

smoothened appropriately, $\bar{L} \cap M_{j+1 / 2}^{\prime}$ is a $(k-1)$-dimensional smcs of $M_{j+1 / 2}^{\prime}$. Since $F^{-1}([j-1 / 2, j+1 / 2]) \backslash\left(U \times D_{p_{i}^{j}}^{n-j}\right)$ after smoothing along the corner is diffeomorphic to $[0,1] \times M_{j-1 / 2}$, where the flow $\varphi_{t}$ corresponds to the flow in the direction of $[0,1], \bar{L} \cap M_{j+1 / 2}^{\prime}$ is diffeomorphic to $\bar{L} \cap M_{j-1 / 2}$. Hence $\bar{L} \cap M_{j-1 / 2}$ is a $(k-1)$-dimensional smcs of $M_{j-1 / 2}$.

Since $\bar{L} \cap M_{k-1 / 2}$ is a union of attaching spheres $S_{p_{i}^{k}}^{k-1}\left(i=1, \ldots, c_{k}\right), \bar{L} \cap M_{j+1 / 2}$ is a $(k-1)$-dimensional smes of $M_{j+1 / 2}$ for $i=k-1, \ldots, 0$.

Let $L=L_{p}^{s}$ be the stable manifold of the critical point $p$ of index $k$. The stable manifold $L$ is diffeomorphic to $\mathbb{R}^{k}$ and the restriction $\varphi_{t} \mid L$ of the flow $\varphi_{t}$ is conjugate to the radial contraction $\psi_{t}$ on $\mathbb{R}^{k}$ defined by $\psi_{t}\left(x_{1}, \ldots, x_{k}\right)=e^{-t}\left(x_{1}, \ldots, x_{k}\right)$. First we embed $\mathbb{R}^{k}$ in $D^{k}$ such that the ray from the origin corresponds to the radial ray in $\operatorname{int}\left(D^{k}\right)$. Let $i: L \rightarrow D^{k}$ denote the embedding. Then we see that the identity map $i(L) \rightarrow L$ does not extend to a continuous map $D^{k} \rightarrow \bar{L}$ in general.

In order to define the map $h: D^{k} \rightarrow \bar{L}$, we use the construction in the above lemmas. For a subset $A$ of $D^{k}$, we write $R(A)$ the radial saturation of $A$, that is the union of the radial segments of length 1 from the origin 0 passing through the points of $A$. 
Proof of Proposition 6.2. We are going to construct the $k$-dimensional compact submanifold $B_{j}$ of $D^{k}$ with boundary such that

$$
B_{k} \subset B_{k-1} \subset \cdots \subset B_{1} \subset B_{0}=D^{k}
$$

and the homeomorphisms

$$
h_{j}: B_{j} \longrightarrow \bar{L} \cap F^{-1}([j-1 / 2, k+1 / 2]) \quad(j=k, \ldots, 0),
$$

such that $h_{j} \mid\left(B_{j} \cap \operatorname{int}\left(D^{k}\right)\right)$ is a diffeomorphism onto $L \cap F^{-1}([j-1 / 2, k+1 / 2])$.

First, for $L=L_{p}^{s}, L \cap M_{k-1 / 2}$ is a $(k-1)$-dimensional sphere which is the attaching sphere $S_{p}^{k-1}$ bounding the core disk $D_{p}^{k}$. Put $B_{k}=i\left(D_{p}^{k}\right) \subset D^{k}$, and we define $h_{k}: B_{k} \rightarrow \bar{L}$ to be $i^{-1}$.

Secondly, we look at the finite set $S_{p}^{k-1} \cap S_{p_{i}^{k-1}}^{n-k}$. The cone $C_{p_{i}^{k-1}}\left(S_{p}^{k-1} \cap S_{p_{i}^{k-1}}^{n-k}\right)$ is contained in $\bar{L}$ and we take the closed disk neighborhood $\bar{U}_{i}$ of $S_{p}^{k-1} \cap S_{p_{i}^{k-1}}^{n-k}$ in $S_{p}^{k-1}$ given by Lemma 6.3 such that $U_{i} \times C_{p_{j}^{k-1}}\left(S_{p}^{k-1} \cap S_{p_{i}^{k-1}}^{n-k}\right)$ is a neighborhood of $C_{p_{j}^{k-1}}\left(S_{p}^{k-1} \cap S_{p_{i}^{k-1}}^{n-k}\right)$ in $\bar{L}$. Then we take the radial saturation $R\left(i\left(\bar{U}_{i}\right)\right)$ in $D^{k}$. The part $R\left(i\left(\bar{U}_{i}\right)\right) \backslash \operatorname{int}\left(i\left(D_{p}^{k}\right)\right)$ is diffeomorphic to $i\left(\bar{U}_{i}\right) \times[0,1]$, where $i\left(\bar{U}_{i}\right) \times\{0\} \subset \partial D^{k}$ and $i\left(\bar{U}_{i}\right) \times\{1\}=i\left(\bar{U}_{i}\right)$. Then we define

$$
h_{k}^{\prime}: i\left(\bar{U}_{i}\right) \times[0,1] \longrightarrow \bar{U}_{i} \times C_{p_{j}^{k-1}}\left(S_{p}^{k-1} \cap S_{p_{i}^{k-1}}^{n-k}\right)
$$

by $h_{k}^{\prime}(x, t)=\left(i^{-1}(x), t\right)$, where $t$ is the parameter of the cone such that $t=0$ corresponds the vertex. Then we take the union $i\left(D_{p}^{k}\right) \cup \bigcup_{i=1}^{c_{k-1}} R\left(i\left(\bar{U}_{i}\right)\right)$ and add a neighborhood of $\bigcup_{i=1}^{c_{k-1}} i\left(\partial \bar{U}_{i}\right)$ to obtain a smooth manifold $B_{k}^{\prime}$ in $D^{k}$. On the other hand, we take the union

$$
D_{p}^{k} \cup \bigcup_{i=1}^{c_{k}-1} \bar{U}_{i} \times C_{p_{j}^{k-1}}\left(S_{p}^{k-1} \cap S_{p_{i}^{k-1}}^{n-k}\right)
$$

and add a neighborhood of $\bigcup_{i=1}^{c_{k-1}} \partial \bar{U}_{i}$ to obtain the subset $A_{k} \subset \bar{L}$. There is a continuous map $h_{k}^{\prime \prime}: B_{k}^{\prime} \rightarrow A_{k} \subset \bar{L}$ extending $h_{k}$ such that $h_{k}^{\prime \prime} \mid\left(B_{k}^{\prime} \cap \operatorname{Int}\left(D^{k}\right)\right)$ is a diffeomorphism onto $L \cap A_{k}$. Since $\bar{L} \cap F^{-1}([k-3 / 2, k+1 / 2]) \backslash A_{k}$ is invariant under the flow $\varphi_{t}$ and the flow $\varphi_{t}$ on

$$
F^{-1}([k-3 / 2, k+1 / 2]) \backslash \bigcup_{i=1}^{c_{k}-1} U_{i} \times D_{p_{i}^{k-1}}^{n-k+1}
$$

is conjugate to the flow on $[0,1] \times M_{k-3 / 2}$ in the direction of $[0,1]$, we can perform the following construction. We take a collar neighborhood $\partial B_{k}^{\prime} \times[0,1]$ of $\partial B_{k}^{\prime}$ in 
$D^{k} \backslash \operatorname{int}\left(B_{k}^{\prime}\right)$ and let $B_{k-1}$ be the union of $B_{k}^{\prime}$ and its collar neighborhood. Using the flow $\varphi_{t}$, we can construct a continuous map

$$
h_{k-1}: B_{k-1} \longrightarrow \bar{L} \cap F^{-1}([k-3 / 2, k+1 / 2])
$$

such that $h_{k-1} \mid\left(B_{k-1} \cap \operatorname{int}\left(D^{k}\right)\right)$ is a diffeomorphism onto $L \cap F^{-1}([k-3 / 2, k+$ $1 / 2])$. We may arrange that $B_{k-1}$ is star-shaped with respect to $0 \in D^{k}$ in such a way that $\partial B_{k-1}$ and radial segments from 0 to points of $\partial D^{k}$ are transverse.

Thirdly, assume that we have constructed the $k$-dimensional compact submanifold $B_{j+1}$ of $D^{k}$ with boundary and the homeomorphism

$$
h_{j+1}: B_{j+1} \longrightarrow \bar{L} \cap F^{-1}([j+1 / 2, k+1 / 2])
$$

such that $h_{j+1} \mid\left(B_{j+1} \cap \operatorname{int}\left(D^{k}\right)\right)$ is a diffeomorphism onto $L \cap F^{-1}([j+1 / 2, k+$ $1 / 2])$ and $B_{j+1}$ is star-shaped with respect to 0 . Then $\bar{L} \cap M_{j+1 / 2}$ is a $(k-1)$ dimensional smcs of $M_{j+1 / 2}$ and the belt spheres $S_{p_{i}^{j}}^{n-j-1}\left(\subset M_{j+1 / 2}\right)$ are transverse to $\bar{L} \cap M_{j+1 / 2}\left(i=1, \ldots c_{j}\right)$. Hence $\bar{L} \cap S_{p_{i}^{j}}^{n-j-1}$ is a $(k-j-1)$-dimensional smcs of $S_{p_{i}^{j}}^{n-j-1}$. The cone $C_{p_{i}^{j}}\left(\bar{L} \cap S_{p_{i}^{j}}^{n-j-1}\right)$ is contained in $\bar{L}$ and we take the closed disk neighborhood $\bar{U}_{i} \subset D_{p_{i}^{j}}^{j}$ of $p_{i}^{k-1}$ given by Lemma 6.3 such that $U_{i} \times C_{p_{i}^{j}}(\bar{L} \cap$ $\left.S_{p_{i}^{j}}^{n-j-1}\right)$ is a neighborhood of $C_{p_{i}^{j}}\left(\bar{L} \cap S_{p_{i}^{j}}^{n-j-1}\right)$ in $\bar{L}$. We look at $\left(h_{j+1}\right)^{-1}(\bar{L} \cap$ $\left.S_{p_{i}^{j}}^{n-j-1}\right)$ and its closed neighborhood

$$
\bar{V}_{i}^{j+1}=\left(h_{j+1}\right)^{-1}\left(\bar{U}_{i} \times\left(\bar{L} \cap S_{p_{i}^{j}}^{n-j-1}\right)\right)
$$

in $\partial B_{j+1}$. Then we take the radial saturation $R\left(\bar{V}_{i}^{j+1}\right)$ in $D^{k}$. This time, the part $R\left(\bar{V}_{i}^{j+1}\right) \backslash \operatorname{int}\left(B_{j+1}\right)$ and $\bar{V}_{i}^{j+1} \times[0,1]$ are not diffeomorphic, but homeomorphic. The reason is that $R\left(\bar{V}_{i}^{j+1}\right) \backslash \operatorname{int}\left(B_{j+1}\right)$ near $\bar{V}_{i}^{j+1} \cap \partial D^{k}$ is a manifold with corner along $\bar{V}_{i}^{j+1} \cap \partial D^{k}$, and there is a homeomorphism $V_{i}^{j+1} \times[0,1] \rightarrow R\left(\bar{V}_{i}^{j+1}\right) \backslash$ $\operatorname{int}\left(B_{j+1}\right)$ such that $V_{i}^{j+1} \times\{0\} \subset \partial D^{k}$ and $V_{i}^{j+1} \times\{0\}=V_{i}^{j+1}$, which straighten the corner along $\left(\bar{V}_{i}^{j+1} \cap \partial D^{k}\right) \times\{0\}$ and is no longer send the radial segments to the direction of $[0,1]$ near $\left(\bar{V}_{i}^{j+1} \cap \partial D^{k}\right) \times\{0\}$. This homeomorphism can be taken to be a diffeomorphism on $V_{i}^{j+1} \times[0,1)$. Then we take the union $B_{j+1} \cup \bigcup_{i=1}^{c_{j}} R\left(\bar{V}_{i}^{j+1}\right)$ and add a neighborhood of $\bigcup_{i=1}^{c_{j}} \partial \bar{V}_{i}^{j+1}$ to obtain a smooth manifold $B_{j+1}^{\prime}$ in $D^{k}$. On the other hand, we take the union

$$
\left(\bar{L} \cap F^{-1}([j+1 / 2, k+1 / 2])\right) \cup \bigcup_{i=1}^{c_{j}} \bar{U}_{i} \times C_{p_{i}^{j}}\left(\bar{L} \cap S_{p_{i}^{j}}^{n-j-1}\right)
$$


and add a neighborhood of $\bigcup_{i=1}^{c_{k-1}} \bar{U}_{i} \times\left(\bar{L} \cap S_{p_{i}^{j}}^{n-j-1}\right)$ to obtain the subset $A_{j+1} \subset \bar{L}$. There is a continuous map $h_{j+1}^{\prime \prime}: B_{j+1}^{\prime} \rightarrow A_{j+1} \subset \bar{L}$ extending $h_{j+1}$ such that $h_{j+1}^{\prime \prime} \mid\left(B_{j+1}^{\prime} \cap \operatorname{Int}\left(D^{k}\right)\right)$ is a diffeomorphism onto $L \cap A_{j+1}$. Since $\bar{L} \cap F^{-1}([j-$ $1 / 2, j+1 / 2]) \backslash A_{j+1}$ is invariant under the flow $\varphi_{t}$ and the flow $\varphi_{t}$ on

$$
F^{-1}([j-1 / 2, j+1 / 2]) \backslash \bigcup_{i=1}^{c_{j}} U_{i} \times D_{p_{i}^{j}}^{n-j}
$$

is conjugate to the flow on $[0,1] \times M_{j-1 / 2}$ in the direction of $[0,1]$, we can perform the following construction. We take a collar neighborhood $\partial B_{j+1}^{\prime} \times[0,1]$ of $\partial B_{j+1}^{\prime}$ in $D^{k} \backslash \operatorname{int}\left(B_{j+1}^{\prime}\right)$ and let $B_{j}$ be the union of $B_{j+1}^{\prime}$ and its collar neighborhood. Using the flow $\varphi_{t}$, we can construct a continuous map $h_{j}: B_{j} \rightarrow \bar{L} \cap F^{-1}([j-1 / 2, k+1 / 2])$ such that $h_{j} \mid\left(B_{j} \cap \operatorname{int}\left(D^{k}\right)\right)$ is a diffeomorphism onto $L \cap F^{-1}([j-1 / 2, k+1 / 2])$. We may arrange that $B_{j}$ is star-shaped with respect to $0 \in D^{k}$ in such a way that $\partial B_{j}$ and radial segments from 0 to points of $\partial D^{k}$ are transverse.

Finally, for $j=0$ in the above construction, we notice that $B_{1}^{\prime}=B_{1} \cup$ $\bigcup_{i=1}^{c_{0}} R\left(\bar{V}_{i}^{1}\right)$ is $D^{k}$ itself and the map $h_{1}^{\prime \prime}: B_{1}^{\prime} \rightarrow A_{1}$ extending $h_{1}$ is the desired map.

\section{References}

[1] R. D. Anderson, The algebraic simplicity of certain groups of homeomorphisms. Amer. J. Math. 80 (1958), 955-963. Zbl 0090.38802 MR 0098145

[2] A. Banyaga, The structure of classical diffeomorphism groups. Math. Appl. 400, Kluwer Academic Publishers Group, Dordrecht 1997. Zbl 0874.58005 MR 1445290

[3] C. Bavard, Longueur stable des commutateurs. L'Enseign. Math. 37 (1991), 109-150. Zbl 0810.20026 MR 1115747

[4] D. Burago, S. Ivanov and L. Polterovich, Conjugation-invariant norms on groups of geometric origin. In Groups of diffeomorphisms, Adv. Stud. Pure Math. 52, Math. Soc. Japan, Tokyo 2008, 221-250. Zbl 05526532 MR 2509711

[5] D. Calegari, scl. MSJ Memoirs 20, Math. Soc. Japan, Tokyo 2009. MR 2527432

[6] S. S. Cairns, A simple triangulation method for smooth manifolds. Bull. Amer. Math. Soc. 67 (1961), 389-390. Zbl 0192.29901 MR 0149491

[7] M. Gromov, Volume and bounded cohomology. Inst. Hautes Études Sci. Publ. Math. 56 (1982), 5-99. Zbl 0516.53046 MR 0686042

[8] D. B. A. Epstein, The simplicity of certain groups of homeomorphisms. Compositio Math. 22 (1970), 165-173. Zbl 0205.28201 MR 0267589

[9] G. M. Fisher, On the group of all homeomorphisms of a manifold. Trans. Amer. Math. Soc. 97 (1960), 193-212. Zbl 0144.22902 MR 0117712 
[10] S. Haller and J. Teichmann, Smooth perfectness through decomposition diffeomorphisms into fiber preserving ones. Ann. Global Anal. Geom. 23 (2003), 53-63. Zbl 1026.58007 MR 1952858

[11] M. Herman, Sur la conjugaison différentiable des difféomorphismes du cercle à des rotations. Inst. Hautes Études Sci. Publ. Math. 49(1979), 5-234. Zbl 0448.58019 MR 0538680

[12] D. Kotschick, Stable length in stable groups. In Groups of diffeomorphisms, Adv. Stud. Pure Math. 52, Math. Soc. Japan, Tokyo 2008, 401-413. Zbl 1188.20028 MR 2509718

[13] F. Laudenbach, On the Thom-Smale complex. Appendix, Astérisque 205 (1992), 219-233.

[14] J. Mather, The vanishing of the homology of certain groups of homeomorphisms. Topology 10 (1971), 297-298. Zbl 0207.21903 MR 0288777

[15] J. Mather, Integrability in codimension 1. Comment. Math. Helv. 48 (1973), 195-233. Zbl 0284.57016 MR 0356085

[16] J. Mather, Commutators of diffeomorphisms I, II, and III. Comment. Math. Helv. 49 (1974), 512-528; 50 (1975), 33-40; and 60 (1985), 122-124. Zbl 0575.58011 MR 0787665

[17] S. Matsumoto and S. Morita, Bounded cohomology of certain groups of homeomorphisms. Trans. Amer. Math. Soc. 94 (1985), 539-544. Zbl 0536.57023 MR 0787909

[18] J. Milnor, Morse theory. Ann. of Math. Stud. 51, Princeton University Press, Princeton, N.J., 1963. Zbl 0108.10401 MR 0163331

[19] J. Milnor, Lectures on the h-cobordism theorem. Princeton University Press, Princeton, N.J., 1965. Zbl 0161.20302 MR 0190942

[20] J. R. Munkres, Elementary differential topology. Ann. of Math. Stud. 54, Princeton University Press, Princeton, N.J., 1963. Zbl 0107.17201 MR 0163320

[21] S. Smale, On gradient dynamical systems. Ann. of Math. (2) 74 (1961), 199-206. Zbl 0136.43702 MR 0133139

[22] R. Thom, Les singularité des application différentiables. Ann. Inst. Fourier (Grenoble) 6 (1956), 43-87. Zbl MR 0087149

[23] W. Thurston, Foliations and groups of diffeomorphisms, Bull. Amer. Math. Soc. 80 (1974), 304-307. Zbl 0295.57014 MR 0339267

[24] D. Trotman, Lectures on real stratification theory. In Singularity theory, World Scientific Publ., Hackensack, N.J., 2007, 139-155. Zbl 1176.58001 MR 2342910

[25] T. Tsuboi, On 2-cycles of $B \operatorname{Diff}\left(S^{1}\right)$ which are represented by foliated $S^{1}$-bundles over $T^{2}$. Ann. Inst. Fourier (Grenoble) 31 (1981), no. 2, 1-59. Zbl 0439.57018 MR 0617240

[26] T. Tsuboi. On the homology of classifying spaces for foliated products. In Foliations, Adv. Stud. Pure Math. 5, North-Holland Publishing Co., Amsterdam; Kinokuniya Company Ltd., Tokyo 1985, 37-120. Zbl 0674.57023 MR 0877328

[27] T. Tsuboi, On the foliated products of class $C^{1}$. Ann. of Math. 130 (1989), 227-271. Zbl 0701.57012 MR 1014925

[28] T. Tsuboi, On the perfectness of groups of diffeomorphisms of the interval tangent to the identity at the endpoints. In Foliations: geometry and dynamics (Warsaw, 2000), World Scientific Publ., River Edge, N.J., 2002, 421-440. Zbl 1012.58006 MR 1882783

[29] T. Tsuboi, On the group of foliation preserving diffeomorphisms. In Foliations 2005, World Scientific Publ., Hackensack, N.J., 2006, 411-430. Zbl MR 2284795 
[30] T. Tsuboi, On the uniform perfectness of diffeomorphism groups. In Groups of diffeomorphisms, Adv. Stud. Pure Math. 52, Math. Soc. Japan, Tokyo 2008, 505-524. Zbl 1183.57024 MR 2509724

[31] T. Tsuboi, On the uniform simplicity of diffeomorphism groups. In Differential geometry, World Scientific Publ., Hackensack, N.J., 2009, 43-55. Zbl 1181.57033 MR 2523489

[32] S. M. Ulam and J. von Neumann, On the group of homeomorphisms of the surface of the sphere (Abstract). Bull. Amer. Math. Soc. 53 (1947), 508.

[33] J. H. C. Whitehead, On $C^{1}$-complexes. Ann. of Math. (2) 41 (1940), 809-824. JFM 66.0955.03 MR 0002545

[34] H. Whitney, The self-intersections of a smooth n-manifold in 2n-space, Ann. of Math. 45 (1944), 220-246. Zbl 0063.08237 MR 0010274

[35] H. Whitney, The singularities of a smooth n-manifold in (2n-1)-space, Ann. of Math. 45 (1944), 247-293. Zbl 0063.08238 MR 0010275

[36] H. Whitney, Local properties of analytic varieties. In Differential and combinatorial topology, Princeton University Press, Princeton, N.J., 1965, 205-244. Zbl 0129.39402 MR 0188486

[37] H. Whitney, Geometric integration theory. Princeton University Press, Princeton, N.J., 1957. Zbl 0083.28204 MR 0087148

Received April 23, 2009; revised May 31, 2009

Takashi Tsuboi, Graduate School of Mathematical Sciences, University of Tokyo, Komaba Meguro, Tokyo 153-8914, Japan

E-mail: tsuboi@ms.u-tokyo.ac.jp 